\title{
The Analysis of 2,5-Dimethoxy-N-(N-methoxybenzyl) phenethylamine (NBOMe) Isomers Using Traditional and Fast Gas Chromatography-Mass Spectrometry
}

\author{
J. Tyler Davidson
}

Follow this and additional works at: https://researchrepository.wvu.edu/etd

\section{Recommended Citation}

Davidson, J. Tyler, "The Analysis of 2,5-Dimethoxy-N-(N-methoxybenzyl) phenethylamine (NBOMe) Isomers Using Traditional and Fast Gas Chromatography-Mass Spectrometry" (2017). Graduate Theses, Dissertations, and Problem Reports. 5438.

https://researchrepository.wvu.edu/etd/5438

This Thesis is protected by copyright and/or related rights. It has been brought to you by the The Research Repository @WVU with permission from the rights-holder(s). You are free to use this Thesis in any way that is permitted by the copyright and related rights legislation that applies to your use. For other uses you must obtain permission from the rights-holder(s) directly, unless additional rights are indicated by a Creative Commons license in the record and/ or on the work itself. This Thesis has been accepted for inclusion in WVU Graduate Theses, Dissertations, and Problem Reports collection by an authorized administrator of The Research Repository @ WVU. For more information, please contact researchrepository@mail.wvu.edu. 


\title{
The Analysis of 2,5-Dimethoxy-N-(N-methoxy- benzyl)phenethylamine (NBOMe) Isomers Using Traditional and Fast Gas Chromatography-Mass Spectrometry
}

\author{
J. Tyler Davidson
}

Thesis submitted to the Eberly College of Arts and Sciences at West Virginia University

in partial fulfillment of the requirements for the degree of

Master of Science in

Forensic and Investigative Science

Glen P. Jackson, Ph.D., Chair

Stephen J. Valentine, Ph.D.

Casper H. Venter, M.S.

Forensic and Investigative Science Department

$$
\begin{gathered}
\text { Morgantown, West Virginia } \\
2017
\end{gathered}
$$

Keywords: Novel Psychoactive Substances, Fast GC, EI Fragmentation Copyright 2017 J. Tyler Davidson 


\title{
Abstract \\ The Analysis of 2,5-Dimethoxy-N-(N-methoxy-benzyl)phenethylamine (NBOMe) Isomers Using Traditional and Fast Gas Chromatography-Mass Spectrometry
}

\author{
J. Tyler Davidson
}

An alarming trend in the field of novel psychoactive substances (NPS) is the emergence of a class of synthetic phenethylamine derivatives known as NBOMes. These substances are low dosage hallucinogenic designer drugs that have become popular for recreational drug use, especially in Europe, Asia, and North America. The hallucinogenic effects of these substances are directly linked to the substitution and arrangement of the generic phenethylamine structure. An outbreak of deaths across the United States has prompted increased legislation to ban their distribution and use, but knowledge about NBOMes, and specifically the positional isomers, is lacking. In addition to identifying particular isomers of NBOMes, seized drug analysts would also benefit from a better understanding of the source of uncertainty in gas chromatography-mass spectrometry (GC-MS) measurements.

The analysis of six NBOMe isomers was conducted using both traditional and fast GCMS for the development of characteristic retention times, retention indices, and fragment ion abundances. One-way analysis of variance (ANOVA) was used to evaluate the significance of different factors on the within-factor to between-factor variance. The factors assessed included positional isomers, concentration, day of analysis, week of analysis, instrument, speed of GC, and the recency of the tune profile. Additionally, principal component analysis (PCA) and canonical discriminant analysis (CDA) were used, respectively, to understand the sources of variance and assess the classification of NBOMe isomers based on the relative ion abundances.

Retention indices provided significantly large inter-isomer retention indices for the six NBOMe isomers studied, which means that all six isomers can be differentiated based on retention time. The measured retention indices established for the six isomers are as follows: 25C-NBOMe ortho $2614 \pm 15$; 25C-NBOMe meta $2666 \pm 13$; 25C-NBOMe para $2692 \pm 13$; 25I-NBOMe ortho $2821 \pm 16$; 25I-NBOMe meta $2877 \pm 15$, and 25I-NBOMe para $2904 \pm 12$.

The fast GC-MS method was twice as rapid as the traditional method, while providing no significant losses in GC resolutions or number of theoretical plates, which provides a compelling case for the future use of fast GC-MS in crime laboratories. In addition, Agilent fast GC provided retention time precision on the order of $0.03 \% \mathrm{RSD}$, compared to $0.10 \% \mathrm{RSD}$ and $1.26 \%$ RSD for Agilent and PerkinElmer traditional GC-MS instruments. These measured precisions suggest that the commonly-adopted retention time match window of $\pm 2 \%$ RSD may actually be too lenient. The CDA classification rate of $99.9 \%$ for the higher concentration samples and $99.5 \%$ for all concentration samples based on the relative ion abundances indicates a great potential for the application of multivariate classification for the identification of compounds with similar chemical structures. Finally, the determination that the effect of the tune profile does not significantly impact the relative ion abundances is significant to the quality assurance programs of crime labs. 


\section{Acknowledgements}

First of all, I would like to express my gratitude to my Principal Investigator Dr. Glen P. Jackson for his continuous support not only of this project, but of my entire educational process. He has always displayed a passion for teaching that has been infectious in nature and truly inspired me to pursue my passions as well. Dr. Jackson has been an excellent advisor along my academic journey and I am glad to be continuing my education under his tutelage for the length of my dissertation.

Besides Dr. Jackson, I would like to thank the rest of my thesis committee for their assistance throughout this project. Whether it was trouble understanding a concept or assistance identifying the problem, they were always available.

I am thankful for my fellow lab members in the Jackson Group, as well as my colleagues in the Forensic and Investigative Science Department. Whether I needed assistance with a problem or just a good laugh, someone was always available.

Last but not least, I would like to thank my family for all of their continued support along this journey. Much of what I have accomplished along my academic career has been a direct result of the values instilled in me and the opportunities afforded to me by my parents. I truly would not be at this point in my academic career without their continued support and encouragement every step of the way.

However, this acknowledgements section would be incomplete without the mention of my best friend and partner in crime, Heather. Even though our educational paths have separated, she is always available to lend an ear or help solve a problem. Her unfailing support and continued encouragement has been essential to the completion of this Master's Thesis. Thank you. 


\section{Table of Contents}

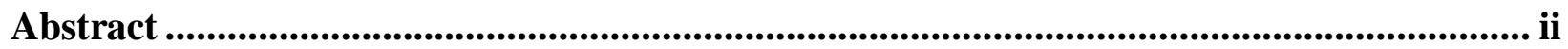

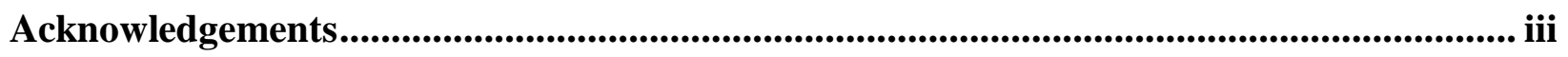

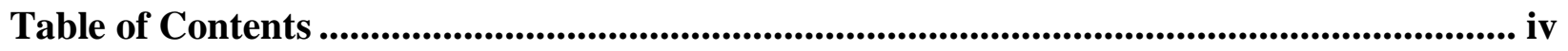

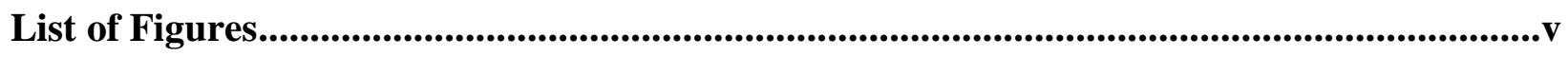

List of Tables: ...................................................................................................... viii

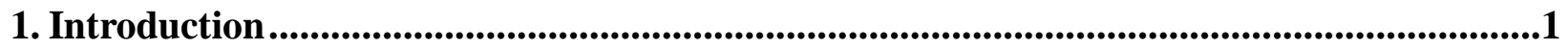

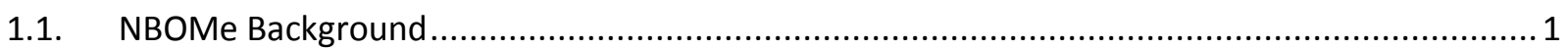

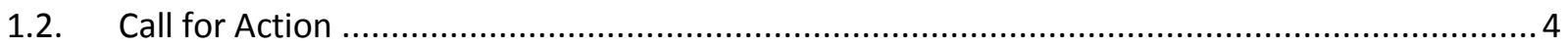

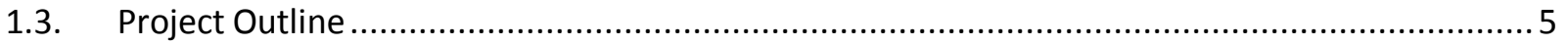

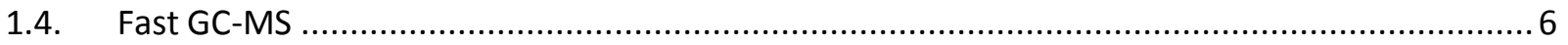

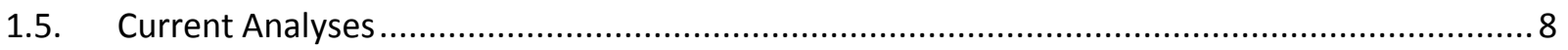

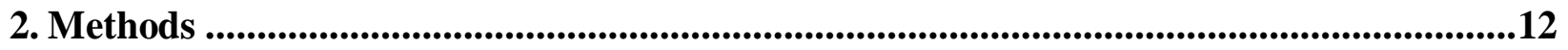

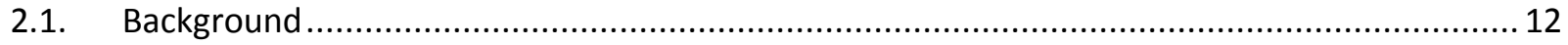

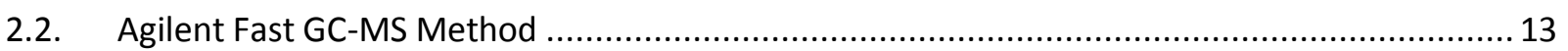

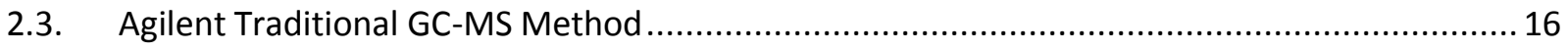

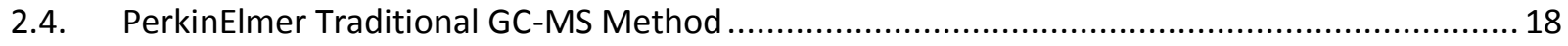

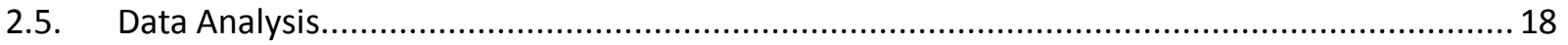

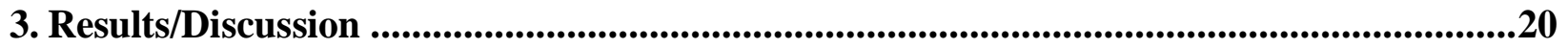

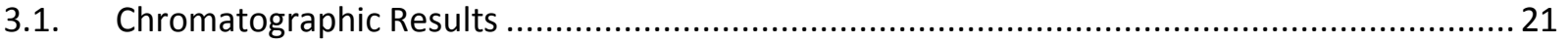

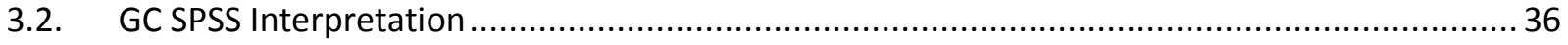

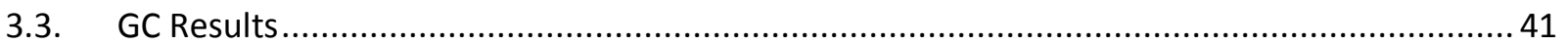

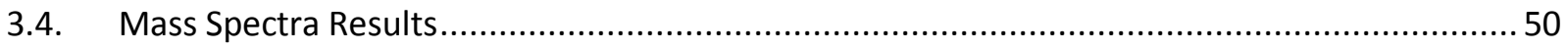

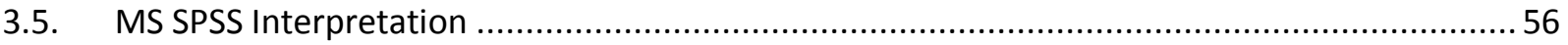

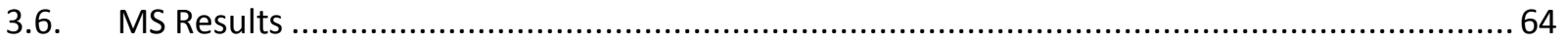

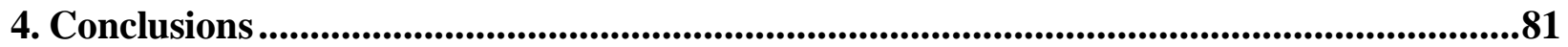

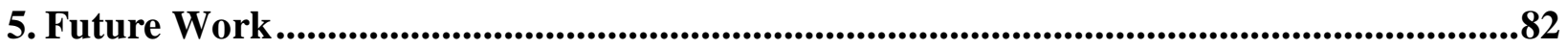

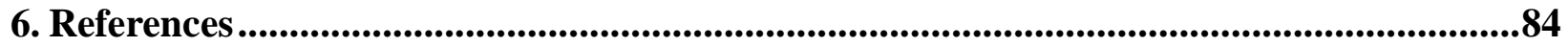




\section{List of Figures:}

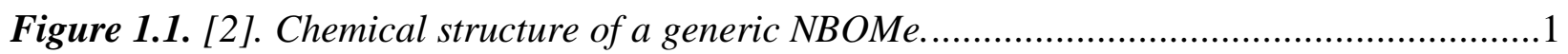

Figure 1.2. [12]. Isomer classification of methoxy substituted phenethylamines. ........................5

Figure 3.1. Total ion chromatogram of a methanol blank analyzed using the Agilent fast GC-MS method.

Figure 3.2. Total ion chromatogram of a methanol blank analyzed using the Agilent traditional GC-MS method. .22

Figure 3.3. Total ion chromatogram of the n-alkane mixture analyzed using the Agilent fast $G C$ MS method.

Figure 3.4. Total ion chromatogram of the n-alkane mixture analyzed using the Agilent traditional GC-MS method.

Figure 3.5. Total ion chromatogram of $12.5 \mathrm{ppm} 25 \mathrm{C}$-NBOMe ortho isomer analyzed using the Agilent fast GC-MS method.

Figure 3.6. Total ion chromatogram of $125 \mathrm{ppm} 25 \mathrm{C}-\mathrm{NBOMe}$ meta isomer analyzed using the Agilent traditional GC-MS method.

Figure 3.7. Total ion chromatogram of 1,250 ppm 25C-NBOMe para isomer analyzed using the Agilent fast GC-MS method.

Figure 3.8. Total ion chromatogram of 1,250 ppm 25I-NBOMe para isomer analyzed using the Agilent traditional GC-MS method. .28

Figure 3.9. Total ion chromatogram of 125 ppm mixture of all six isomers using the Agilent fast GC-MS method.

Figure 3.10. Total ion chromatogram of 125 ppm mixture of all six isomers using the Agilent traditional GC-MS method.

Figure 3.11. Total ion chromatogram of 12.5 ppm mixture of the 25C-NBOMe mixture using the Agilent fast GC-MS method. This sample was $\sim 4$ weeks old and showed contamination peaks of PDMS from the storage vial caps.

Figure 3.12. Total ion chromatogram of 12.5 ppm mixture of the 25I-NBOMe mixture using the Agilent traditional GC-MS method. This sample was $\sim 8$ weeks old and showed contamination peaks of PDMS from the storage vial caps.

Figure 3.13. $Q-Q$ plot of $12.5 \mathrm{ppm} 25 \mathrm{C}-\mathrm{NBOMe}$ ortho isomer demonstrating normality of retention time data.

Figure 3.14. Box and whisker plots for the retention indices corresponding to the PerkinElmer traditional GC-MS (a), Agilent fast GC-MS (b), and the Agilent traditional GC-MS (c).

Figure 3.15. Box and whisker plots for the retention indices broken down by the methodology and the fixed concentration of the sample corresponding to the PerkinElmer traditional GC-MS (a), Agilent fast GC-MS (b), and the Agilent traditional GC-MS (c). The three boxes at each positional isomer are for the 12.5, 125, and 1250 ppm solutions, respectively.

Figure 3.16. Combined (across three concentrations) retention indices results for all methodologies studied. 
Figure 3.17. Combined retention indices results broken down instrumental methodology. Each set of three box and whisker plots corresponds to the PerkinElmer, Agilent fast, and Agilent traditional retention indices results per isomer.

Figure 3.18. 12.5 ppm 25C-NBOMe Agilent fast GC-MS spectra corresponding to 25C-ortho (a), 25C-meta (b), and 25C-para (c).....

Figure 3.19. 125 ppm 25I-NBOMe Agilent traditional GC-MS spectra corresponding to 25Iortho (a), 25I-meta (b), and 25I-para (c).

Figure 3.20. 1,250 ppm 25C-NBOMe PerkinElmer traditional GC-MS spectra corresponding to 25C-ortho (a), 25C-meta (b), and 25C-para (c). .55

Figure 3.21. $Q-Q$ plot of 12.5 ppm 25C-NBOMe ortho isomer demonstrating normality..........57 Figure 3.22. Principal Component Analysis (PCA) showing the natural clustering of the NBOMe isomers (a) and the component correlation matrix showing the relationship between each ion and the NBOMe isomer groupings where function 1 and 2 are estimates of the correlations between each of the variables and the estimated components (b). N=1515 for the entire data set.

Figure 3.23. Canonical Discriminant Analysis (CDA) showing the classification of the NBOMe isomers based on the relative ion abundances (a) and the structure matrix correlation values where function 1 and 2 are the pooled within group correlations between the discriminant variables and the standardized canonical discriminant functions $(b) . N=1515$ for the entire data set.

Figure 3.24. Principal Component Analysis (PCA) showing the natural clustering of the NBOMe isomers (a) and the component correlation matrix showing the relationship between each ion and the NBOMe isomer groupings where function 1 and 2 are estimates of the correlations between each of the variables and the estimated components $(b) . N=1029$ for the premium data set which consisted of the $125 \mathrm{ppm}$ and 1,250 ppm samples.

Figure 3.25. Canonical Discriminant Analysis (CDA) showing the classification of the NBOMe isomers based on the relative ion abundances (a) and the structure matrix correlation values where function 1 and 2 are the pooled within group correlations between the discriminant variables and the standardized canonical discriminant functions (b). N=1029 for the premium data set, which contains the 125 ppm and 1,250 ppm samples.

Figure 3.26. Canonical Discriminant Analysis (CDA) showing the classification of the NBOMe isomers based on the relative ion abundances for only the Agilent fast GC-MS methodology (a) and the structure matrix correlation values where function 1 and 2 are the pooled within group correlations between the discriminant variables and the standardized canonical discriminant functions (b). $N=519$ for the Agilent fast GC-MS entire data set.

Figure 3.27. Canonical Discriminant Analysis (CDA) showing the classification of the NBOMe isomers based on the relative ion abundances for only the Agilent fast GC-MS methodology (a) and the structure matrix correlation values where function 1 and 2 are the pooled within group correlations between the discriminant variables and the standardized canonical discriminant 
functions (b). $N=293$ for the Agilent fast GC-MS premium data set, which contains the $125 \mathrm{ppm}$ and 1,250 ppm samples.

Figure 3.28. 95\% confidence interval for the characteristic ion ratios between the six NBOMe isomers studied. *Indicates statistically significantly different based on t-test results for each $\mathrm{m} / \mathrm{z}$ value between isomers.

Figure 3.29. Box and whisker plots of the relative ion abundances for the four characteristic ion ratios proposed through this research, which are $\mathrm{m} / \mathrm{z}, 150(\mathrm{a}), \mathrm{m} / \mathrm{z} 91$ (b), $\mathrm{m} / \mathrm{z}, 186(\mathrm{c})$, and $\mathrm{m} / \mathrm{z}$ $278(d)$. 


\section{List of Tables:}

Table 1.1. [2]. Reported retention times for the three most common NBOMes by GC-MS

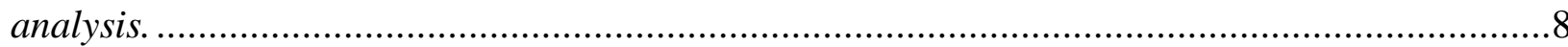

Table 2.1. Composition of stock solutions for Agilent fast GC-MS analysis. ............................13

Table 2.2. Working solutions used for all GC-MS analyses. ..............................................14

Table 2.3. Methodology for the A gilent fast GC-MS analysis..............................................15

Table 2.4. Composition of stock solutions for Agilent traditional GC-MS analysis..................16

Table 2.5. Methodology for the Agilent traditional GC-MS analysis.....................................17

Table 2.6. Methodology for the PerkinElmer traditional GC-MS analysis. ............................18

Table 2.7. Auto integration parameters used for data analysis. ..............................................20

Table 3.1. Agilent Fast GC-MS vs Agilent traditional GC-MS separation efficiency results.......32

Table 3.2. Averaged results for Agilent fast GC-MS vs Agilent traditional GC-MS separation

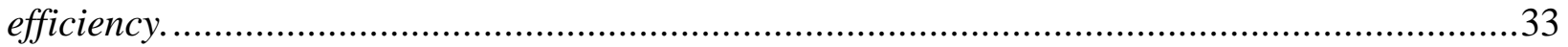

Table 3.3. ANOVA Retention time and retention index at the $95 \%$ confidence level. ................38

Table 3.4. Eta squared retention time, retention index, and peak area results.........................40

Table 3.5. 95\% confidence intervals for the retention indices of the combined data. ................46

Table 3.6. $R S D$ results for the retention time reproducibility....................................................48

Table 3.7. ANOVA results ( $p$ values) for the inter-factor variance and intra-factor variance considering four different fixed factors. 15 different fragment ion abundances (normalized to $\mathrm{m} / \mathrm{z}$

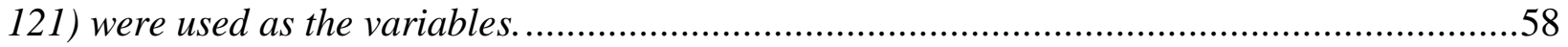

Table 3.8. ANOVA results ( $p$ values) for each analysis method. ..........................................59

Table 3.9. Eta squared ion abundance data. .......................................................................61

Table 3.10. Eta squared ion relative abundance data for each analysis method. ......................62

Table 3.11. 95\% confidence interval characteristic ion ratios...............................................77 


\section{Introduction}

\subsection{NBOMe Background}

2,5-dimethoxy-N-(N-methoxy-benzyl)phenethylamines (NBOMes) are a class of synthetic phenethylamine derivatives, or novel psychoactive substances (NPSs), that have become increasingly popular in Europe, the United States, and Asia [1]. NBOMes are derivatives of the larger " $2 \mathrm{C}$ " class of compounds, so named by Dr. Alexander Shulgin because of the two carbon atoms between the benzene ring and the amino group on the phenethylamine $[1, \underline{2}]$. This 2C structure is common among other classes of drugs, such as amphetamines, catecholamines, cathinones, and many designer drugs since the 1970s. Over time there has been continued substitution to the generic phenethylamine structure leading to an abundance of $2 \mathrm{C}$ designer drugs. The substitutions made to the generic phenethylamine structure are responsible for the different physiological and psychological effects of 2C designer drugs [3]. The generic NBOMe structure is shown in Figure 1.1, where $R_{2}, R_{3}$, and $R_{4}$ indicate the location of the methoxy group for each isomer and $\mathrm{R}_{1}$ is the location of the additional functional group, typically a lipophilic substituent [4].

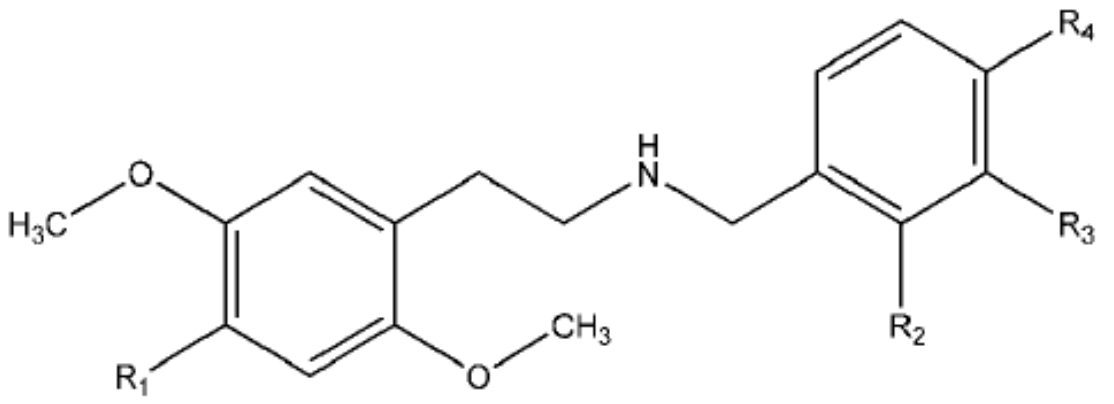

Figure 1.1. [2]. Chemical structure of a generic NBOMe. 
NBOMes are low dosage hallucinogenic drugs, which has made them popular for recreational drug use [1]. The Drug Enforcement Agency (DEA) recognized this trend and in November of 2013 placed three NBOMes temporarily into the Schedule I category of the Controlled Substances Act. The three NBOMes scheduled were 25I-, 25C-, and 25B-NBOMe, the three most common NBOMes on the market at the time []․ This temporary scheduling was then extended in November of 2015 []]. The "25" represents the 2,5-dimethoxy substitutions and the I, C, and B letters represent the different halogen substituents.

The first reported synthesis of an NBOMe was in 2003 by Dr. Ralf Heim of the University of Berlin who synthesized 25I-NBOMe as a pharmacological tool to study the 5- $\mathrm{HT}_{2 \mathrm{~A}}$ receptor $[\underline{1}, \underline{2}, \underline{5}]$. The $5-\mathrm{HT}_{2 \mathrm{~A}}$ receptor is a serotonin G-Coupled Protein Receptor (GPCR) that has been closely linked to complex behaviors such as working memory and cognitive processes. The activation of this receptor occurs through the interaction with an agonist, which results in significant behavioral toxicity and provides hallucinogenic and stimulatory effects [4]. The 5- $\mathrm{HT}_{2 \mathrm{~A}}$ receptor is also known to be responsible for the hallucinogenic effects of LSD [1, $\underline{4}$. However, LSD is only a partial agonist of the $5-\mathrm{HT}_{2 \mathrm{~A}}$ receptor, which means NBOMes actually produce a stronger hallucinogenic effect $[4,7]$. Also, the hallucinogenic effects of NBOMes are altered by the different substituents attached to their common structure $[\underline{3}, \underline{4}, \underline{8}]$.

NBOMes first became available over the internet in 2011 and were marketed as either legal highs or research chemicals that were not for human consumption $[\underline{1}, \underline{4}]$. NBOMes are usually distributed as a powder or diluted to sub-milligram doses and laced into blotter paper [2]]. NBOMes are often complexed with hydroxypropyl-beta-cyclodextrin (HPBCD) to increase their bioavailability $[1, \underline{9}]$. The blotter paper is frequently marked with identifying artwork and then cut into tiny squares. These blotter paper squares are then administered sublingually to gain 
direct entry into the blood vessels under the tongue or placed against the cheek to allow for absorption through the cheek membranes in a method known as buccal administration. The price of a single $500 \mu \mathrm{g}$ hit can be as low as $\$ 5[1]$.

Other forms of abuse include nasal insufflation of powders, intravenous injection, nasal absorption of liquid solutions, and consumption of food laced with NBOMes [ㄷ]. The mode of administration will affect the time for effects to be felt (10-30 mins) and the duration of the effects [10]. Sublingual and buccal administration generally takes longer for the effects to be felt, but the duration of the high increases [10]. The typical total duration of the effects is between four and eight hours [4].

Abusers of NBOMes are typically young males between the ages of 14-29 years. When under the influence, abusers typically present symptoms of a serotonin-like syndrome, including violent physical and mental episodes that can be so extreme that they ultimately lead to death $[1, \underline{2}]$. Other adverse health effects of NBOMe usage include tachycardia, hypertension, agitation, aggression, visual and auditory hallucinations, seizures, hyperpyrexia, clonus, elevated white blood cell count, elevated creatine kinase, metabolic acidosis, rhabdomyolysis, and acute kidney failure [11]. Whereas there have been no studies published on the potency of NBOMes, several illicit drug-related internet websites recommend sub-milligram doses [2]. 25I-NBOMe is the most-widely abused NBOMe and the most potent. It is sold under the names of NBOMe and Smiles and anecdotal reports indicate powder doses of 50-250 $\mu \mathrm{g}$ and blotter paper doses of 500-800 $\mu \mathrm{g}$ to be administered sublingually, by nasal insufflation, or applied to the buccal cavity $[1, \underline{3}]$. 


\subsection{Call for Action}

Between June of 2011 and June of 2013, 959 reports containing 25I, 25C, or 25B NBOMes from across 35 states were reported to the National Forensic Laboratory Information System (NFLIS) []․ According to NFLIS, there were no submissions containing any type of NBOMe prior to June of 2011. Furthermore, the United States Customs and Border Protection data indicates bulk quantities of 25I, 25C, and 25B NBOMes have been seized from shipments originating from overseas, particularly from Asian countries []. Not only are NBOMes becoming increasingly available within the United States, but 11 states have implicated some combination of 25I, 25C, or 25B NBOMes in the death of at least 17 individuals. The reported deaths were categorized as 14 deaths due to acute toxicity and three deaths associated with behavior due to NBOMe abuse [5]. Despite an increasing effort to ban NBOMe use and trafficking in many countries, intoxications and fatalities have continued to increase worldwide [4].

The increasing prevalence of NBOMe intoxications and fatalities has necessitated an increased emphasis on the characterization and identification of different NBOMes. For example, there is a general lack of information about isomer differentiation of specific NBOMes. In this project, the analysis of the ortho, meta, and para isomers of both 25I- and 25C-NBOMe was conducted to contribute to the advancement of knowledge about NBOMes. The reason characterization of isomers is so important is because the substitution and arrangement of substituents dictates the hallucinogenic potency of these drugs [5]. The trends/rules learned in this work could be compared to isomer differentiation of other NPS substances, which could then provide more general rules for interpreting spectra from unknown substances in the future. 
The location of the methoxy substituent on the phenethylamine moiety relative to the amino group determines the classification of the isomer, as seen below, in Figure 1.2.

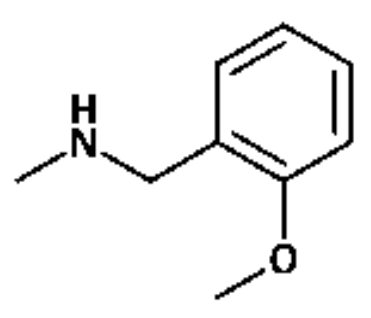

Ortho

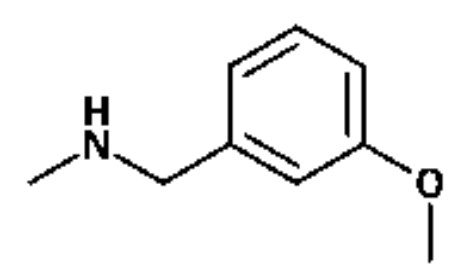

Meta

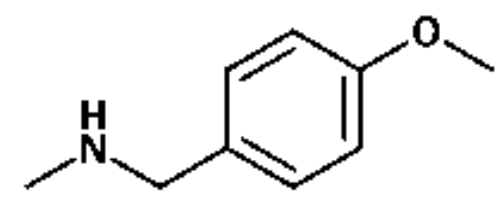

Para

Figure 1.2. [12]. Isomer classification of methoxy substituted phenethylamines.

\subsection{Project Outline}

In this project, the analysis of NBOMe isomers was conducted with Gas

Chromatography-Mass Spectrometry (GC-MS). The instruments used for this analysis were an Agilent Technologies 7890B GC which was linked to an Agilent Technologies 5977A MS detector and a PerkinElmer Clarus 680 GC linked to a PerkinElmer Clarus SQ8S MS detector. The development of characteristic retention times and retention indices for each isomer, as well as the variability of the relative ion abundances, was assessed by analyzing standards of each isomer on different instruments and on different days.

One-way analysis of variance (ANOVA) was used to evaluate the significance of different factors on the within-factor to between-factor variance. The factors assessed included positional isomers, concentration, day of analysis, week of analysis, instrument, speed of GC, and the recency of the tune profile. The variables assessed included the retention time, retention index, relative ion abundances and measured ion ratios.

Principal components analysis (PCA) and canonical discriminant analysis (CDA) were used to identify the sources of variance and perform an assessment of the classification of NBOMe isomers based on the relative ion abundance data. 


\subsection{Fast GC-MS}

In an extension of this study, experiments were repeated using fast GC-MS instead of traditional GC-MS. Fast GC-MS involves the use of a shorter and narrower column, faster oven temperature ramp rates, and higher carrier gas velocities to achieve faster separations, without scarifying resolution [13]. The interest in fast GC-MS is primarily driven by the desire to reduce the cost per analysis through higher throughput, better utilization of high-cost instrumentation, and the need for fewer analysts [14].

Since the creation of capillary columns in 1958 , there has been an interest in speeding up the time required for GC analysis. In fact, in 1960 the main topic at the international symposium on GC was speed-related aspects of separation. A presentation by Desty and Goldup showed that a one-minute isothermal separation of 10 peaks in a $15 \mathrm{~m}$ x $125 \mu \mathrm{m}$ column with the use of hydrogen as the carrier gas was possible [14]. From this point on there would be further investigation into principles of fast GC, ultimately leading to the current state of knowledge [15].

The theory behind fast GC is based on increasing the rate of chromatographic separation, while maintaining the same level of chromatographic separation efficiency. Equation 1 shows the retention time $t_{R}$ as a function of the column length $L$, the carrier gas velocity $u$, and the retention factor $\mathrm{k}[13]$.

$$
t_{R}=\frac{L(k+1)}{\mathrm{u}}
$$

The Van Deemter equation is shown in Equation 2 and it relates the height equivalent to a theoretical plate (HETP), which is an expression for the efficiency of a chromatographic separation where $\mathrm{A}$ is the eddy diffusion, $\mathrm{B}$ is the longitudinal diffusion, $\mathrm{C}_{\mathrm{s}}$ is the resistance to mass transfer for the stationary phase, $\mathrm{C}_{\mathrm{m}}$ is the resistance to mass transfer for the mobile phase, and $\mathrm{u}$ is the average velocity of the mobile phase. 


$$
\operatorname{HETP}=A+\frac{B}{\mathrm{u}}+C_{s} \mathrm{u}+C_{m} \mathrm{u}
$$

The eddy diffusion term describes the multiple paths effect, where the movement of solute molecules through the stationary phase of the analytical column occurs through different random paths, which does not apply to capillary columns. Longitudinal diffusion describes the extent to which analytes diffuse axially (in the dimension of the detector) in the mobile phase and therefore broaden while they are on the column. The stationary phase resistance to mass transfer accounts for the time required for a species to diffuse radially through the solid stationary phase, and the mobile phase resistance to mass transfer accounts for the radial diffusion of analyte molecules into or out of the mobile phase [16].

An evaluation of the retention time and Van Deemter equations indicates that the best methods to decrease the retention time, while maintaining chromatographic separation efficiency, involve decreasing the longitudinal diffusion (B) or decreasing both the resistance to mass transfer of the mobile phase $\left(\mathrm{C}_{\mathrm{m}}\right)$ and resistance to mass transfer of the stationary phase $\left(\mathrm{C}_{\mathrm{s}}\right)$. To accomplish this task, the internal diameter of the column was reduced, the thickness of the stationary phase was decreased, and the total length of the column was shortened. 


\subsection{Current Analyses}

There are currently no reliable immunoassay rapid drug screenings available for the detection of NBOMes. Instead, analysis is conducted primarily with Gas Chromatography-Mass Spectrometry (GC-MS) or Liquid Chromatography-Mass Spectrometry (LC-MS) []ㅡ. Using GC-MS, Casale and Hays of the DEA performed an analysis on NBOMes in their basic form. Table 1.1, seen below, includes the reported retention times for the three most common NBOMes analyzed with GC-MS according to Casale and Hays [2].

Table 1.1. [2]. Reported retention times for the three most common NBOMes by GC-MS analysis.

\begin{tabular}{|l|c|c|c|}
\hline Isomer & 25I (min) & 25C (min) & 25B (min) \\
\hline 2-methoxy (ortho) & 29.31 & 26.91 & 28.00 \\
\hline 3-methoxy (meta) & 29.80 & 27.54 & 28.60 \\
\hline 4-methoxy (para) & 30.20 & 27.85 & 29.90 \\
\hline
\end{tabular}

The methodology of Casale and Hays included the use of a $30 \mathrm{~m}$ x $0.25 \mathrm{~mm} \times 0.25 \mu \mathrm{m}$ $100 \%$ dimethylpolysiloxane column with an initial oven temperature of $100^{\circ} \mathrm{C}$ and a temperature ramp rate of $6^{\circ} \mathrm{C} / \mathrm{min}$ to a final temperature of $300^{\circ} \mathrm{C}$. The slower temperature ramp rate combined with the higher boiling points resulted in the lengthy retention times of approximately 30 minutes of the NBOMe isomers, with the added benefit that all 9 isomers could be baseline resolved [2].

According to Casale and Hays, the elution of the NBOMe isomers was always in the ortho, meta, para order. The peaks of particular interest in their analyses were the peaks at $\mathrm{m} / \mathrm{z}$ values of 150,121 , and 91 . The peak at $m / z, 121$ is the base peak for all isomers and corresponded to the cleavage of the benzyl moiety, the peak at $m / z 150$ is due to $\alpha$-cleavage of the phenethylamine moiety, and the peak at $m / z 91$ is the common tropylium ion. The abundance of the tropylium ion was always the highest for the ortho isomer and the 
molecular ion peak was quite small in all circumstances, ranging between 0.05 and 1.0 percent abundance [2]. The development of characteristic ion ratios between several of the peaks of interest were determined for a few of the NBOMe compounds. Specifically, the characteristic ion ratios between the peaks at $\mathrm{m} / \mathrm{z} 150$ and $\mathrm{m} / \mathrm{z} 91$ for 25C-NBOMe were determined to be 1.6 (ortho), 6.1 (meta), and 5.3 (para) [2].

Lum, Brophy, and Hibbert [17] conducted an analysis of different types of NBOMes using GC-MS to study the fragmentation of NBOMes derivatized with heptafluorobutyric anhydride (HFBA). Their method used a $12 \mathrm{~m} \times 0.20 \mathrm{~mm} \times 0.33 \mu \mathrm{m}$ column, an initial oven temperature of $80^{\circ} \mathrm{C}$ (hold for $1.50 \mathrm{~min}$ ), a temperature ramp rate of $50^{\circ} \mathrm{C} / \mathrm{min}$ to $270{ }^{\circ} \mathrm{C}$ (hold for $1.67 \mathrm{~min}$ ) and an additional temperature ramp of $35^{\circ} \mathrm{C} / \mathrm{min}$ to a final temperature of $290^{\circ} \mathrm{C}$ for $2.70 \mathrm{~min}$. The column diameter and length are smaller than normal, and are a form of fast GC-MS, but the article did not specifically discuss this element of their research. The use of a quicker temperature ramp did allow for retention times less than 10 minutes. However, this group was only separating different NBOMes, not their positional isomers. Derivatization with HFBA enabled the molecular ion to be more easily identified, and the characteristic McLafferty arrangement products were seen at $m / z 242$ and $m / z$ 244. In similar work, Sánchez Robayo et al. used a $30 \mathrm{~m} \times 250 \mu \mathrm{m} \times 0.25 \mu \mathrm{m}$ VF-5MS column to separate five different isomers and identify the components of blotter paper from street samples []].

The majority of analyses of NBOMes conducted via LC-MS focus specifically on the quantitation of NBOMes in biological fluids and tissues. For example, Poklis et al.[18] used LC-MS to quantitate the concentration of 25I-NBOMe in brain tissue, bile, liver tissue, and gastric contents of a patient who had died from NBOMe usage. The analysis was conducted with HPLC/MS/MS using a reverse phase (C8) $100 \AA$ x $100 \mathrm{~mm}$ x $2.0 \mathrm{~mm}$ column [18]. The results 
of this analysis are shown below in Table 1.2. The concentration of 25I-NBOMe throughout the different specimens analyzed varies drastically, with much larger concentrations being found in the bile and liver than in the heart or peripheral blood. It was noted that the peripheral blood and urine concentrations were within the range of previously reported concentrations of intoxicated patients [18].

Table 1.2. [18]. Concentration of 25I-NBOMe.

\begin{tabular}{|l|l|}
\hline \multicolumn{1}{|c|}{ Specimen } & \multicolumn{1}{|c|}{$\begin{array}{c}\text { Concentration } \\
(\mathbf{p g} / \mathbf{m L} \text { or pg/g) }\end{array}$} \\
\hline Heart blood & 410 \\
\hline Peripheral blood & 405 \\
\hline Urine & 2860 \\
\hline Vitreous humor & 99 \\
\hline Brain & 2780 \\
\hline Liver & 5640 \\
\hline Bile & 12,100 \\
\hline Gastric contents & ND \\
\hline
\end{tabular}

Using HPLC/MS/MS, Poklis et al. developed a method to determine the concentration of 25I-NBOMe and 2CC-NBOMe in human serum [19]. The resulting method used a conventional $100 \AA$ x 100 mm x 2.0 mm column and a gradient elution from 80\% A (deionized water with 10 $\mathrm{mM}$ ammonium acetate and $0.1 \%$ formic acid) to $80 \% \mathrm{~B}$ (methanol). The total separation time was on the order of five minutes. The method returned concentrations within $\pm 15 \%$ of the expected value, produced a linear regression correlation of $0.996 \pm 0.002$, and a limit of quantitation of $10 \mathrm{pg} / \mathrm{mL}$ for 25I-NBOMe. Uchiyama et al. used LC-MS to identify different NBOMes that were present in liquid samples purchased off the streets of Japan [20], [21].

Alternative methods for the analysis of NBOMes have also sought to simplify and decrease the cost of the analysis of NBOMes. For example, the analysis of blotter paper with ATR-FTIR was performed by Jose' Neto [22]. He showed a rapid analysis method to detect a 
series of novel psychoactive substances, including 25C-and 25I-NBOMe that were laced into blotter paper. The technique involved the analysis of both sides of the blotter paper and the application of chemometric analysis to discriminate the illicit substance from the cellulose background. The entire process took approximately two minutes from start to finish. Because each blotter paper was treated as a mixture of paper and one or more NPSs, one downside to this analysis was that the FTIR discriminating power was reduced and the technique was no longer considered a Category A test according to SWGDRG recommendations [22]. The result of this study was that 25B-, 25C-, or 25I-NBOMe was found to be present on $50.6 \%$ of the blotter papers analyzed, indicating the overwhelming presence of these substances on the blotter paper market.

A second alternative analysis was conducted by Duffau et al. [23] in which HPTLC with confirmatory GC-MS analysis was used for the detection of 25C-NBOMe in blotter papers from Chile. This technique was a simple and robust method that was validated according to SWGTOX recommendations for the quantitation of 25C-NBOMe present in blotter papers.

Confirmatory GC-MS analysis was also conducted with an Agilent Technologies $6890 \mathrm{~N}$ GC linked to an Agilent Technologies 5973 B MS. The parameters for this analysis were an initial temperature of $75^{\circ} \mathrm{C}$ for one minute, a $25^{\circ} \mathrm{C} / \mathrm{min}$ ramp rate to $280{ }^{\circ} \mathrm{C}$ with a two-minute hold time, a $1 \mathrm{~mL} / \mathrm{min}$ helium carrier gas flow rate, and splitless injection. The results were that a rapid qualitative and quantitative analysis of 25C-NBOMe using HPTLC was developed and validated for the use of blotter paper analysis in Chile [23]. This method produced reliable results for a relatively small cost and helped to indicate the disturbing trend of broad variations in 25C-NBOMe concentrations found on blotter papers. 
The final example of an alternative method for the analysis of NBOMes was by Salomone et al. [24] in which the determination of designer drugs from hair samples was examined. In this study an UHPLC-MS/MS method was developed for the determination of 31 stimulant and psychedelic substituted phenethylamines from hair samples. Due to the absence of reference standards for the metabolites of many of these substances and the complex nature in which they present in biological fluids such as urine, the use of hair samples was proposed. The sample preparation prior to analysis consisted of $25 \mathrm{mg}$ of hair being washed twice with dichloromethane and methanol, drying of the hair, and trimming of the hair to 1-2 $\mathrm{mm}$ segments. An internal standard was added, a dilution with $1.5 \mathrm{~mL}$ of methanol was performed and an incubation at $55^{\circ} \mathrm{C}$ for 15 hours, without stirring, occurred. Finally, $1 \mu \mathrm{L}$ of the organic phase was removed for the UHPLC-MS/MS analysis. The inclusion of this technique reduced the time to carryout comprehensive toxicological screenings and increased overall laboratory productivity [24].

\section{Methods}

\subsection{Background}

This study employed two different GC-MS instruments, a PerkinElmer Clarus 680 GC and an Agilent Technologies 7890B GC. The Agilent instrument was operated in both traditional and fast GC mode, while the PerkinElmer instrument was only operated in traditional GC mode. The fast GC column installed was a VF-5MS column of dimensions $10 \mathrm{~m}$ x $0.15 \mathrm{~mm}$ x $0.15 \mu \mathrm{m}$ purchased through Agilent Technologies. This new column was chosen based on its use in the article by Sánchez Robayo et al. [8] and the composition of its stationary phase, which was equivalent to that of the SLB-5MS column demonstrated in the article by Elie et al. [25, 26]. The 
fast GC column was conditioned as per the manufacturer's instructions by venting into the GC oven before installing into the transfer line of the mass spectrometer. This procedure prevents excess column bleed entering the ion source and thereby reduces noise/background and helps to extend the lifetime of the filament.

\subsection{Agilent Fast GC-MS Method}

The six stock solutions that were used to create the working solutions analyzed for the fast GC-MS portion of this research were created as described in Table 2.1. Each stock solution was made by transferring approximately $1 \mathrm{mg}$ of each NBOMe isomer to a $3-\mathrm{mL}$ silanized glass vial and then adding $1 \mathrm{~mL}$ of methanol with an adjustable pipette.

Table 2.1. Composition of stock solutions for Agilent fast GC-MS analysis.

\begin{tabular}{|l|l|}
\hline 25C-NBOMe ortho isomer & $1.10 \mathrm{mg} / 1 \mathrm{~mL}$ methanol \\
\hline 25C-NBOMe meta isomer & $0.96 \mathrm{mg} / 1 \mathrm{~mL}$ methanol \\
\hline 25C-NBOMe para isomer & $1.01 \mathrm{mg} / 1 \mathrm{~mL}$ methanol \\
\hline 25I-NBOMe ortho isomer & $1.25 \mathrm{mg} / 1 \mathrm{~mL}$ methanol \\
\hline 25I-NBOMe meta isomer & $1.18 \mathrm{mg} / 1 \mathrm{~mL}$ methanol \\
\hline 25I-NBOMe para isomer & $1.00 \mathrm{mg} / 1 \mathrm{~mL}$ methanol \\
\hline
\end{tabular}

From these stock solutions, 24 working solutions, as seen in Table 2.2, were created for the fast GC-MS portion of this research. The working solutions involving mixtures of NBOMe isomers were brought up to $1 \mathrm{~mL}$ with methanol after the addition of the required volumes of the appropriate NBOMe isomers. The concentrations of the three working solutions were $12.5 \mathrm{ppm}$, 125 ppm, and 1,250 ppm, respectively.

Each solution was analyzed at least twice a week for a month, depending on instrument availability. When the samples were not actively being analyzed, they were stored in a refrigerator and a new cap was added after each injection to prevent solvent evaporation. For quality control, a chloroform blank, methanol blank, and an n-alkane ladder were run at the 
beginning and end of each sequence. Between each sample analysis, a solvent rinse with both chloroform and methanol was used to clean the injection syringe. All samples were analyzed in a randomized order and a blank was run after each 1,250 ppm solution to ensure no carryover was occurring. The randomization process was carried out using Microsoft Excel Version 14. The "=Randbetween $(1,24) "$ command was used to generate randomized numbers between 1 and 24 , which corresponded to the total number of samples analyzed per run. The column containing the sample names was then sorted based on the column containing the random numbers generated by the above function.

Table 2.2. Working solutions used for all GC-MS analyses.

\begin{tabular}{|l|l|}
\hline 1 & 25C-ortho $12.5 \mathrm{ppm}$ \\
\hline 2 & 25C-ortho $125 \mathrm{ppm}$ \\
\hline 3 & 25C-ortho $1,250 \mathrm{ppm}$ \\
\hline 4 & 25C-meta $12.5 \mathrm{ppm}$ \\
\hline 5 & 25C-meta125 ppm \\
\hline 6 & 25C-meta $1,250 \mathrm{ppm}$ \\
\hline 7 & 25C-para $12.5 \mathrm{ppm}$ \\
\hline 8 & 25C-para $125 \mathrm{ppm}$ \\
\hline 9 & 25C-para $1,250 \mathrm{ppm}$ \\
\hline 10 & 25C-mix $125 \mathrm{ppm}$ \\
\hline 11 & 25C-mix $1,250 \mathrm{ppm}$ \\
\hline 12 & 25I-ortho $12.5 \mathrm{ppm}$ \\
\hline 13 & 25I-ortho $125 \mathrm{ppm}$ \\
\hline 14 & 25I-ortho $1,250 \mathrm{ppm}$ \\
\hline 15 & 25I-meta $12.5 \mathrm{ppm}$ \\
\hline 16 & 25I-meta125 ppm \\
\hline 17 & 25I-meta $1,250 \mathrm{ppm}$ \\
\hline 18 & 25I-para $12.5 \mathrm{ppm}$ \\
\hline 19 & 25I-para $125 \mathrm{ppm}$ \\
\hline 20 & 25I-para $1,250 \mathrm{ppm}$ \\
\hline 21 & 25I-mix $125 \mathrm{ppm}$ \\
\hline 22 & 25I-mix $1,250 \mathrm{ppm}$ \\
\hline 23 & All six mix $125 \mathrm{ppm}$ \\
\hline 24 & All six mix $1,250 \mathrm{ppm}$ \\
\hline
\end{tabular}

*Note: Randomized per analysis 
The methodology used for this analysis was optimized from the initial parameters described in previously published works $[26,27]$ to produce the shortest total run time without sacrificing chromatographic separation efficiency. The main parameters that were adjusted to reach this optimized result were the split ratio, initial oven temperature, oven ramp rate, and the carrier gas flow rate. The goal of this optimization was to have baseline resolved peaks with as much chromatographic separation as possible, while maintaining rapid analysis.

In accordance with fast GC theory, the use of a larger split ratio limited the amount of solute entering the column and helped prevent overloading the column. Also, the temperature ramp rate was increased to allow for faster boiling point separation. Finally, the initial and final temperatures were set to allow for efficient chromatographic separation of the NBOMe isomers over the range they eluted from the column. The final Agilent fast GC-MS parameters are shown below in Table 2.3.

Table 2.3. Methodology for the Agilent fast GC-MS analysis.

\begin{tabular}{|l|l|}
\hline Sample injection & $1 \mu \mathrm{L}$ \\
\hline Split ratio & $100: 1$ \\
\hline Carrier gas & Helium \\
\hline Flow rate & $1 \mathrm{~mL} / \mathrm{min}$ \\
\hline Scan region $(\mathrm{m} / \mathrm{z})$ & $25-500$ \\
\hline Initial temperature & $150{ }^{\circ} \mathrm{C}$ \\
\hline Hold time & $\mathrm{N} / \mathrm{A}$ \\
\hline Ramp rate & $25^{\circ} \mathrm{C} / \mathrm{min}$ \\
\hline Final temperature & $280^{\circ} \mathrm{C}$ \\
\hline Final hold time & $1 \mathrm{~min}$ \\
\hline Solvent delay & $0.5 \mathrm{~min}$ \\
\hline Electron ionization energy & $70 \mathrm{eV}$ \\
\hline Ion source temperature & $250{ }^{\circ} \mathrm{C}$ \\
\hline Transfer line temperature & $280^{\circ} \mathrm{C}$ \\
\hline Total separation time & $6.20 \mathrm{~min}$ \\
\hline
\end{tabular}

To assess the effect of the tune profile on the generated mass spectra, an autotune was performed on the morning before each analysis and each sample was analyzed twice, once with 
the tune file collected on day 1 of the trial and again with the updated daily tune profile. The Agilent fast GC-MS analysis was performed over a four-week period of time and the resulting data was collected, extracted, and analyzed using Excel (Microsoft Corporation) Version 14 and SPSS Version 24.

\subsection{Agilent Traditional GC-MS Method}

The next phase of this research used a traditional HP-5 $30 \mathrm{~m} \times 0.25 \mathrm{~mm} \times 0.25 \mu \mathrm{m}$ column in the Agilent GC-MS, which was conditioned as per the manufacturer's instructions by venting into the GC oven. Table 2.4 contains the six stock solutions that were used to create the working solutions analyzed for both the Agilent traditional GC-MS analysis and the PerkinElmer traditional GC-MS analysis. Again, 24 working solutions were created, as seen previously in Table 2.2, and were randomized before every analysis. However, for the traditional analyses there were 24 working solutions created for each traditional instrument setup (Agilent and PerkinElmer).

Table 2.4. Composition of stock solutions for Agilent traditional GC-MS analysis.

\begin{tabular}{|l|l|}
\hline 25C-NBOMe ortho isomer & $1.20 \mathrm{mg} / 1 \mathrm{~mL}$ methanol \\
\hline 25C-NBOMe meta isomer & $1.40 \mathrm{mg} / 1 \mathrm{~mL}$ methanol \\
\hline 25C-NBOMe para isomer & $1.20 \mathrm{mg} / 1 \mathrm{~mL}$ methanol \\
\hline 25I-NBOMe ortho isomer & $1.40 \mathrm{mg} / 1 \mathrm{~mL}$ methanol \\
\hline 25I-NBOMe meta isomer & $1.50 \mathrm{mg} / 1 \mathrm{~mL}$ methanol \\
\hline 25I-NBOMe para isomer & $2.80 \mathrm{mg} / 1 \mathrm{~mL}$ methanol \\
\hline
\end{tabular}

Each solution was analyzed at least twice a week for eight weeks, depending on instrument availability. When the samples were not actively being analyzed, they were stored in a refrigerator and a new cap was added after each injection to prevent the evaporation of solvent. For quality control, a chloroform blank, methanol blank, and an n-alkane ladder were run at the beginning and end of each sequence. Between each sample analysis, a solvent rinse with both 
chloroform and methanol was used to clean the injection syringe. All samples were analyzed in a randomized order and a blank was run after each 1,250 ppm solution to ensure no carryover was occurring.

The Agilent traditional GC-MS parameters are shown below in Table 2.5. Once again, these parameters were developed through an optimization process to maximize the chromatographic separation, while minimizing the total run time. The initial parameters were based off of the work by Casale and Hays [2].

Table 2.5. Methodology for the Agilent traditional GC-MS analysis.

\begin{tabular}{|l|l|}
\hline Sample injection & $1 \mu \mathrm{L}$ \\
\hline Split ratio & $40: 1$ \\
\hline Carrier gas & Helium \\
\hline Flow rate & $1 \mathrm{~mL} / \mathrm{min}$ \\
\hline Scan region $(\mathrm{m} / \mathrm{z})$ & $25-500$ \\
\hline Initial temperature & $150^{\circ} \mathrm{C}$ \\
\hline Hold time & $1 \mathrm{~min}$ \\
\hline Ramp rate & $15^{\circ} \mathrm{C} / \mathrm{min}$ \\
\hline Final temperature & $280^{\circ} \mathrm{C}$ \\
\hline Final hold time & $3 \mathrm{~min}$ \\
\hline Solvent delay & $2 \mathrm{~min}$ \\
\hline Electron ionization energy & $70 \mathrm{eV}$ \\
\hline Ion source temperature & $250^{\circ} \mathrm{C}$ \\
\hline Transfer line temperature & $280^{\circ} \mathrm{C}$ \\
\hline Total separation time & $12.67 \mathrm{~min}$ \\
\hline
\end{tabular}




\subsection{PerkinElmer Traditional GC-MS Method}

The column for the PerkinElmer traditional analysis that was already present in this instrument was used for the analysis. This column was a ZB-5MS column of dimensions $20 \mathrm{~m} \mathrm{x}$ $0.18 \mathrm{~mm} \times 0.18 \mu \mathrm{m}$. Again, the same autotune procedure was followed as previously described. The PerkinElmer traditional GC-MS parameters are shown below in Table 2.6. Once again, these parameters were developed through an optimization process to maximize the chromatographic separation, while minimizing the total run time. The initial parameters for this analysis were again based off of the work by Casale and Hays [2].

Table 2.6. Methodology for the PerkinElmer traditional GC-MS analysis.

\begin{tabular}{|l|l|}
\hline Sample injection & $1 \mu \mathrm{L}$ \\
\hline Split ratio & Splitless \\
\hline Carrier gas & Helium \\
\hline Flow rate & $1 \mathrm{~mL} / \mathrm{min}$ \\
\hline Scan region $(\mathrm{m} / \mathrm{z})$ & $25-500$ \\
\hline Initial temperature & $150^{\circ} \mathrm{C}$ \\
\hline Hold time & $1 \mathrm{~min}$ \\
\hline Ramp rate & $15^{\circ} \mathrm{C} / \mathrm{min}$ \\
\hline Final temperature & $280^{\circ} \mathrm{C}$ \\
\hline Final hold time & $3 \mathrm{~min}$ \\
\hline Solvent delay & $2 \mathrm{~min}$ \\
\hline Electron ionization energy & $70 \mathrm{eV}$ \\
\hline Ion source temperature & $250^{\circ} \mathrm{C}$ \\
\hline Transfer line temperature & $280^{\circ} \mathrm{C}$ \\
\hline Total separation time & $12.67 \mathrm{~min}$ \\
\hline
\end{tabular}

\subsection{Data Analysis}

A large sample size was required so that the intra-day, inter-day, and inter-instrument variability could be determined precisely. Variables that were assessed included the retention time, retention index, and relative ion abundance. As previously mentioned, the effect of the tune profile was also monitored through a continually updated method and a method with a fixed tune 
profile. The resulting data was collected, extracted, and analyzed using Microsoft Excel Version 14 and SPSS Version 24.

One-way analysis of variance (ANOVA) was performed to evaluate the significance of different factors on the within-factor to between-factor variance. Factors that were assessed included positional isomers, concentration, day of analysis, week of analysis, instrument, the tune profile, and the speed of GC. Variables that were assessed included the retention time, retention index, and relative ion abundances. ANOVA is a collection of statistical models that are used to analyze the differences between group means based on the analysis of variance [28]. The observed variance in a particular variable is partitioned into components attributable to different sources of variation. ANOVA is useful for comparing means to determine if there is a statistically significant difference between them.

Principal components analysis (PCA) and canonical discriminant analysis (CDA) were used to show natural clustering and classification of the relative ion abundance data. PCA is a data reduction technique that is commonly used to find natural patterns within a data set by maximizing the total variation [29]. CDA is a multivariate discriminant analysis technique that determines how best to separate or discriminate between two or more groups, by maximizing the between-group variance and minimizing the within-group variance, given quantitative measurements of several variables for the individuals that make up the groups [30].

The data analysis began with the auto integration of each raw file to obtain the retention times and peak areas. The auto integration feature was set according to the parameters shown in Table 2.7 to reduce any bias associated with manually integrating peaks. The retention times and peak areas were extracted into Excel. 
Table 2.7. Auto integration parameters used

for data analysis.

\begin{tabular}{|l|l|}
\hline Initial area reject & 1 \\
\hline Initial peak width & 0.020 \\
\hline Shoulder detection & Off \\
\hline Initial threshold & 18.0 \\
\hline
\end{tabular}

The n-alkane mixture enabled the retention indices associated with each isomer to be calculated [31]. The equation, shown below in Equation 3, involves the retention time of the unknown $\left(t_{r(x)}\right)$, the retention time of the next n-alkane lower in retention time $\left(t_{r_{(n)}}\right)$, the retention time of the next n-alkane higher in retention time, and the number of carbon atoms in the next n-alkane lower in retention time (n).

$$
\mathrm{RI}=100 \mathrm{n}+100\left(\frac{t_{r(x)}-t_{r_{(n)}}}{t_{r_{(n+1)}}-t_{r_{(n)}}}\right)
$$

The extraction of the raw mass spectral data was performed via the 3D data extraction option through Agilent's data analysis software. The extraction was performed by selecting the scan number corresponding to the peak apex and extracting the abundance of every ion between $\mathrm{m} / z 18$ and $\mathrm{m} / z$ 500. The results of this extraction were saved as a .CSV file. Each .CSV file was opened and its contents were transferred to Excel for workup.

\section{Results/Discussion}

The analytical results are broken down into two main sections. The first section describes the GC results, which include exemplar chromatograms, SPSS analysis and interpretations, and numerical results. The second section describes the MS results, which include exemplar mass spectra, SPSS analysis and interpretations, and numerical results. 


\subsection{Chromatographic Results}

Figure 3.1 is a methanol blank that was analyzed with Agilent fast GC-MS. The peaks at 1.2, 1.6, and 2.2 minutes share fragment ions at $\mathrm{m} / \mathrm{z} 73,147,207,221$, and 281, which are characteristic of a homologous series of cyclic polydimethylsiloxanes (PDMS) found in septum bleed [32]. The total run time was 6.20 minutes and no NBOMe compounds were present.

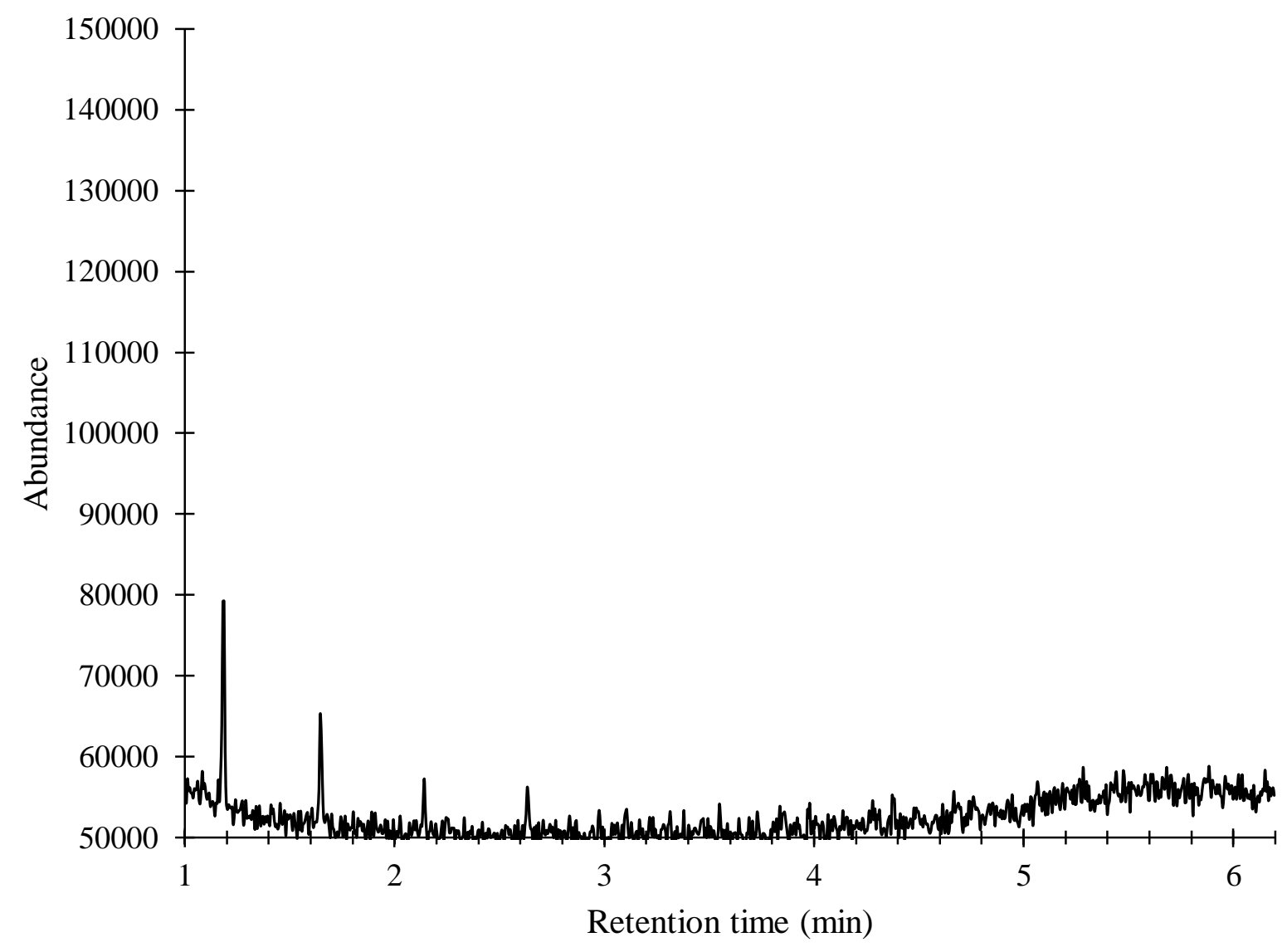

Figure 3.1. Total ion chromatogram of a methanol blank analyzed using the Agilent fast GC-MS method. 
Figure 3.2 shows a methanol blank analyzed by Agilent traditional GC-MS. There were no peaks present in this chromatogram and the baseline increase is consistent with the evaporation of the stationary phase from the analytical column [33].

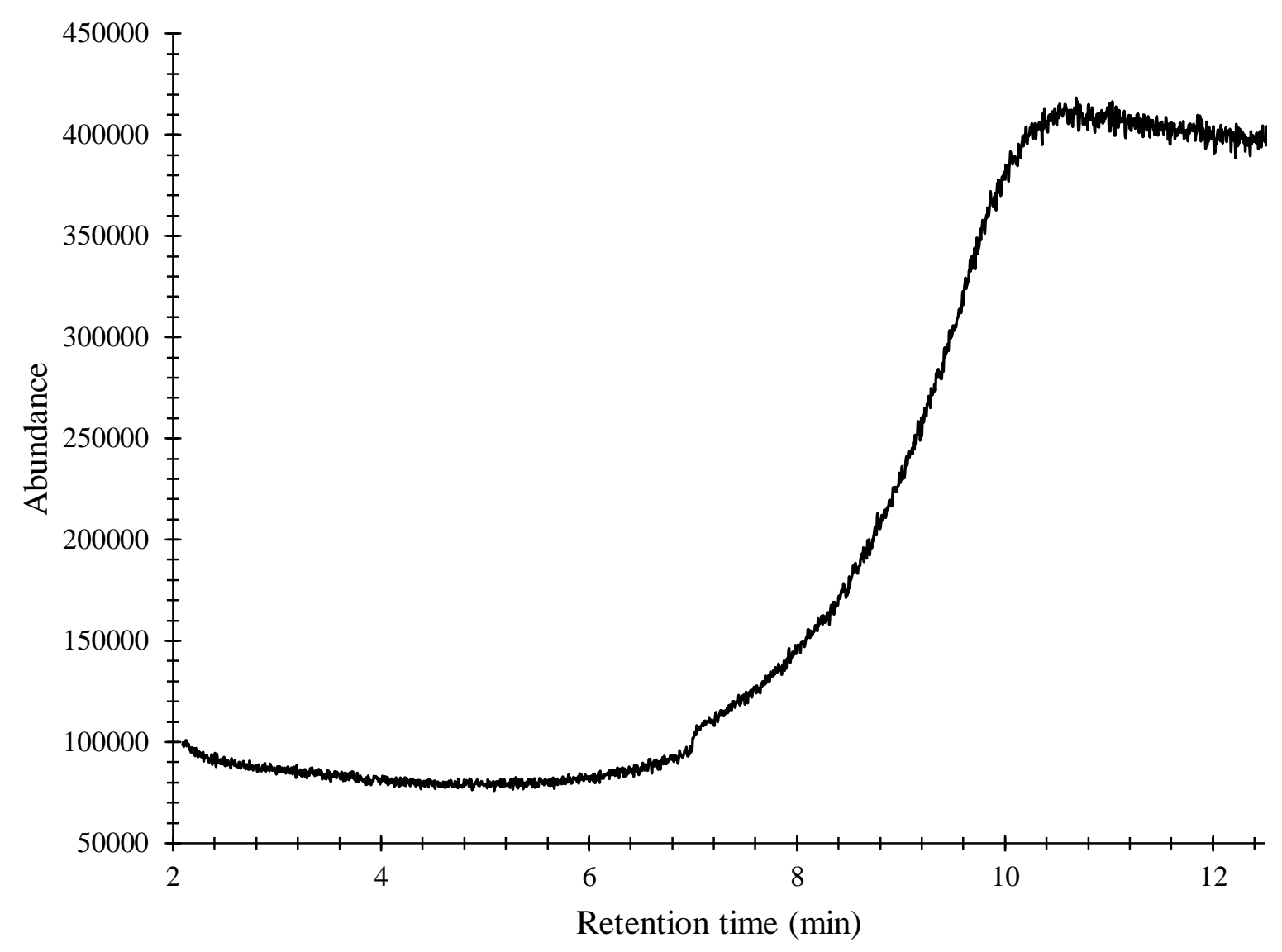

Figure 3.2. Total ion chromatogram of a methanol blank analyzed using the Agilent traditional GC-MS method. 
Figure 3.3 shows an example of a typical n-alkane ladder that was analyzed at the beginning and end of every sequence using the Agilent fast GC-MS method. The elution of nalkanes C14-C30 was performed in less than 6 minutes. The n-alkanes smaller than C14 eluted before the solvent delay at 0.5 minutes. The presence of contamination peaks between the nalkanes was identified. These peaks were identified as PDMS compounds from column or septum bleed due to the presence of ions at $\mathrm{m} / \mathrm{z} 73,147,207,221$ [32].

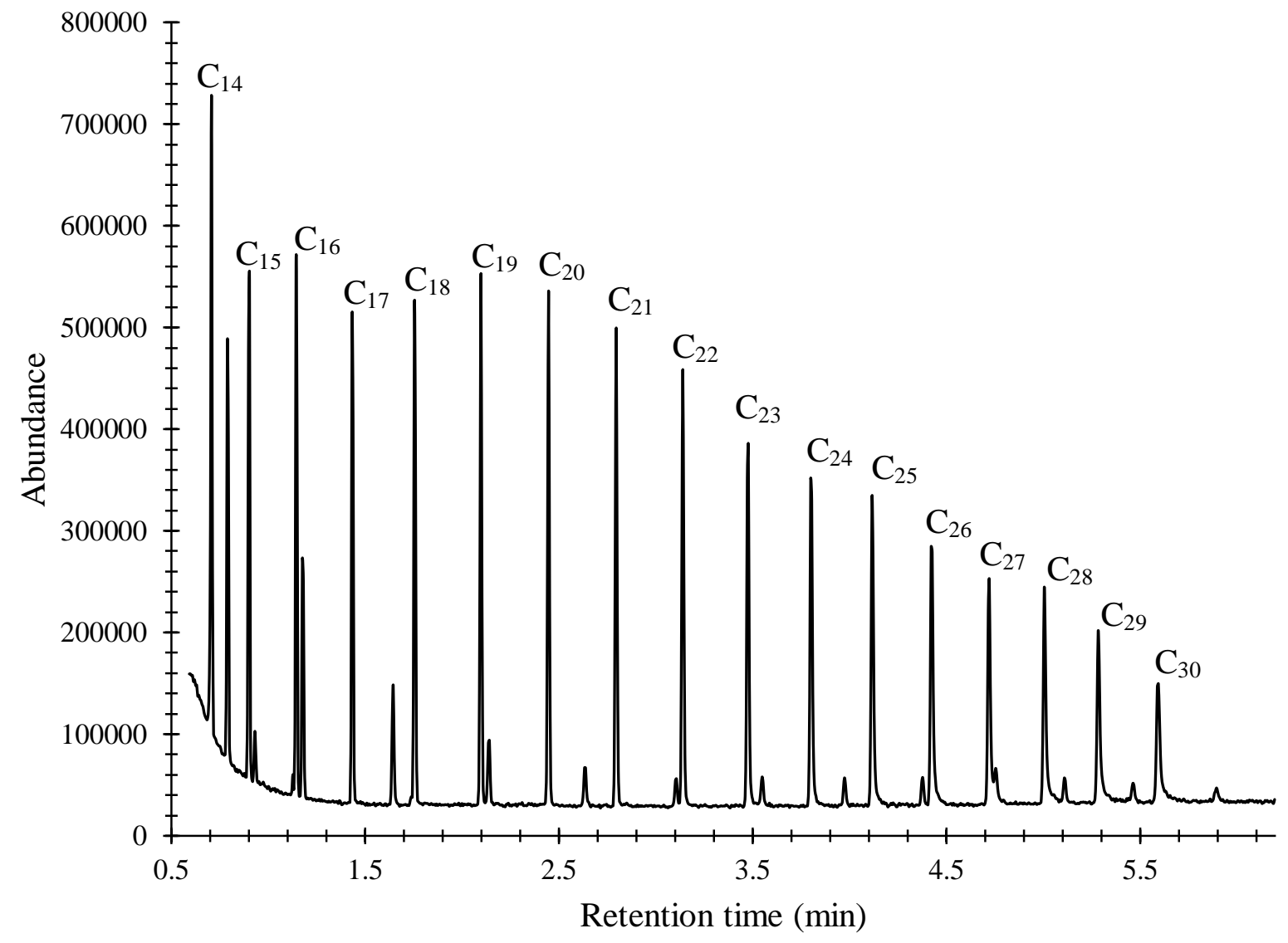

Figure 3.3. Total ion chromatogram of the n-alkane mixture analyzed using the Agilent fast GCMS method. 
Figure 3.4 shows an example of a typical n-alkane ladder that was analyzed at the beginning and end of every sequence using the Agilent traditional GC-MS method. The elution of n-alkanes C14-C30 was performed in less than 13 minutes. The septum bleed peaks were still present, but were lower in abundance than the Agilent fast GC method because of the lower split ratio used in the Agilent traditional GC-MS methodology. In the Agilent injection port, the use of elevated split flows, and the associated higher overall flow rates, seems to carry more septum bleed to the column.

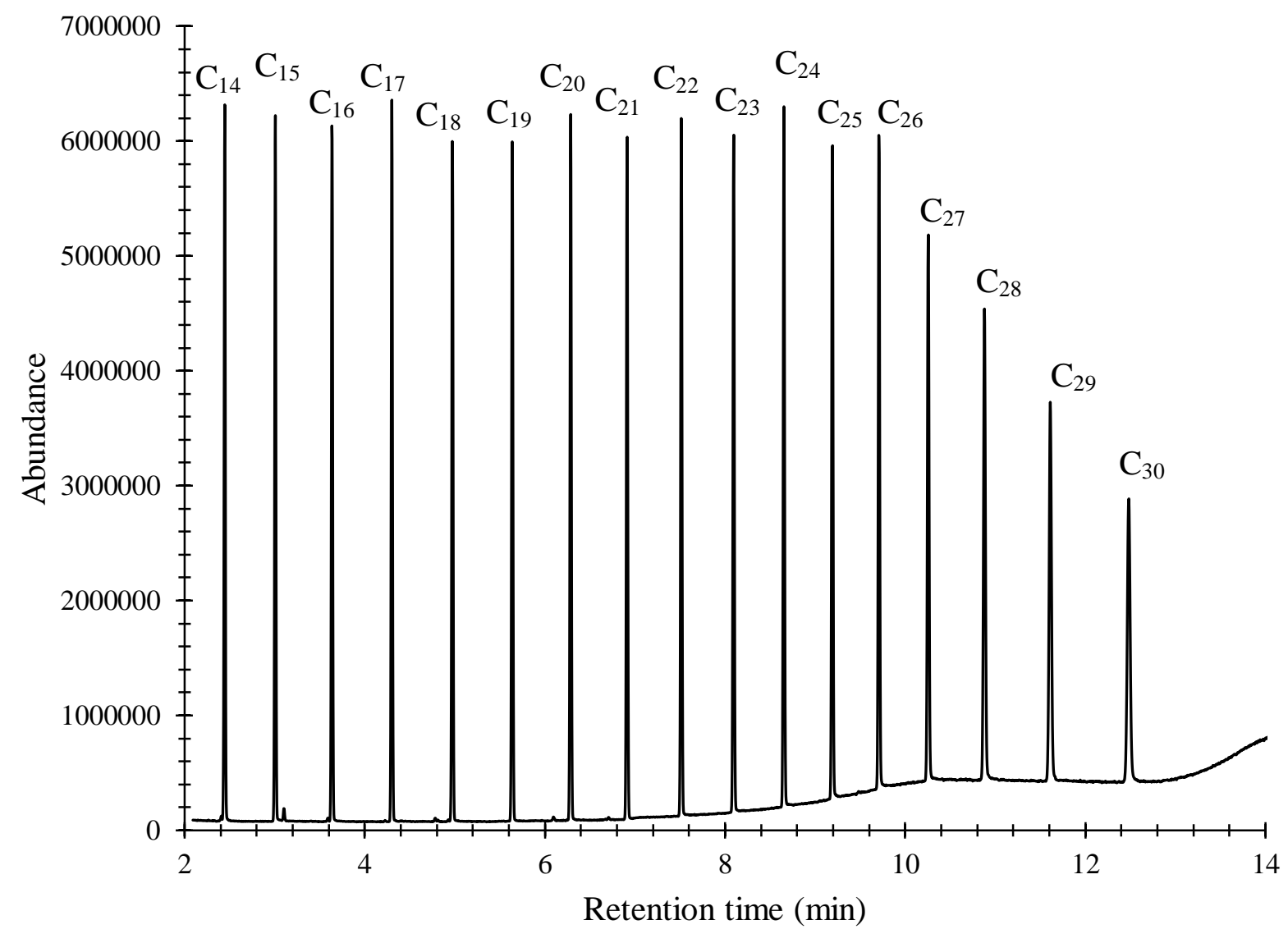

Figure 3.4. Total ion chromatogram of the n-alkane mixture analyzed using the Agilent traditional GC-MS method. 
The chromatogram in Figure 3.5 is a 12.5 ppm 25C-NBOMe ortho isomer analyzed with Agilent fast GC-MS. The only peak present in this chromatogram with a signal to noise ratio greater than 3:1 is the 25C-NBOMe ortho isomer, which eluted at 4.455 minutes. The larger split ratio enabled very narrow peaks to be obtained in the resulting chromatograms, but clearly at the expense of signal to noise.

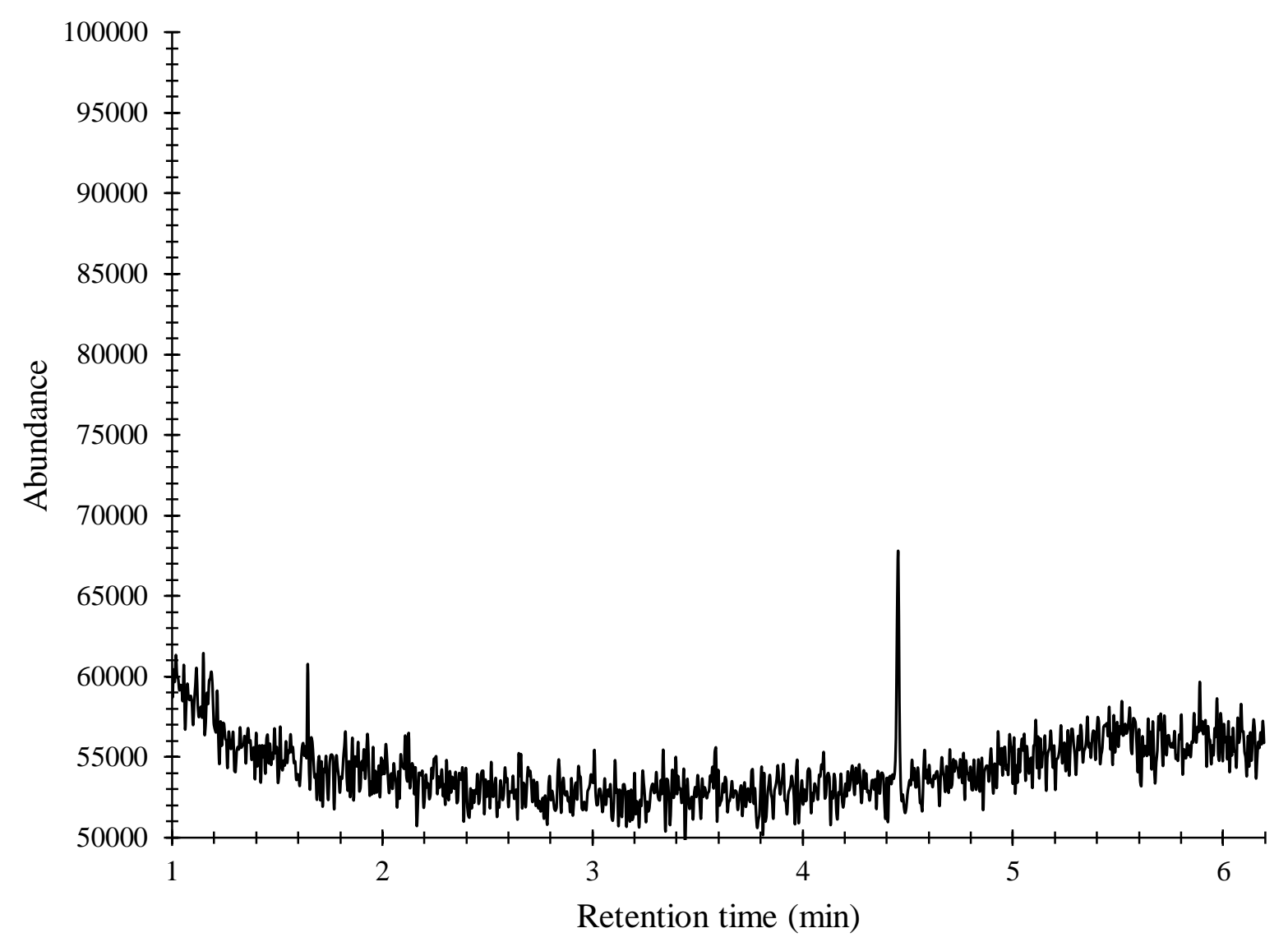

Figure 3.5. Total ion chromatogram of $12.5 \mathrm{ppm}$ 25C-NBOMe ortho isomer analyzed using the Agilent fast GC-MS method. 
The chromatogram in Figure 3.6 is an example of a higher concentration isomer; a 125 ppm 25C-NBOMe meta isomer analyzed with the Agilent traditional GC-MS method. The larger intensity, as compared to Figure 3.5, is due to the combination of a higher concentrated sample and the smaller split ratio utilized in the Agilent traditional GC-MS methodology. The elution of this isomer occurred at 10.152 minutes.

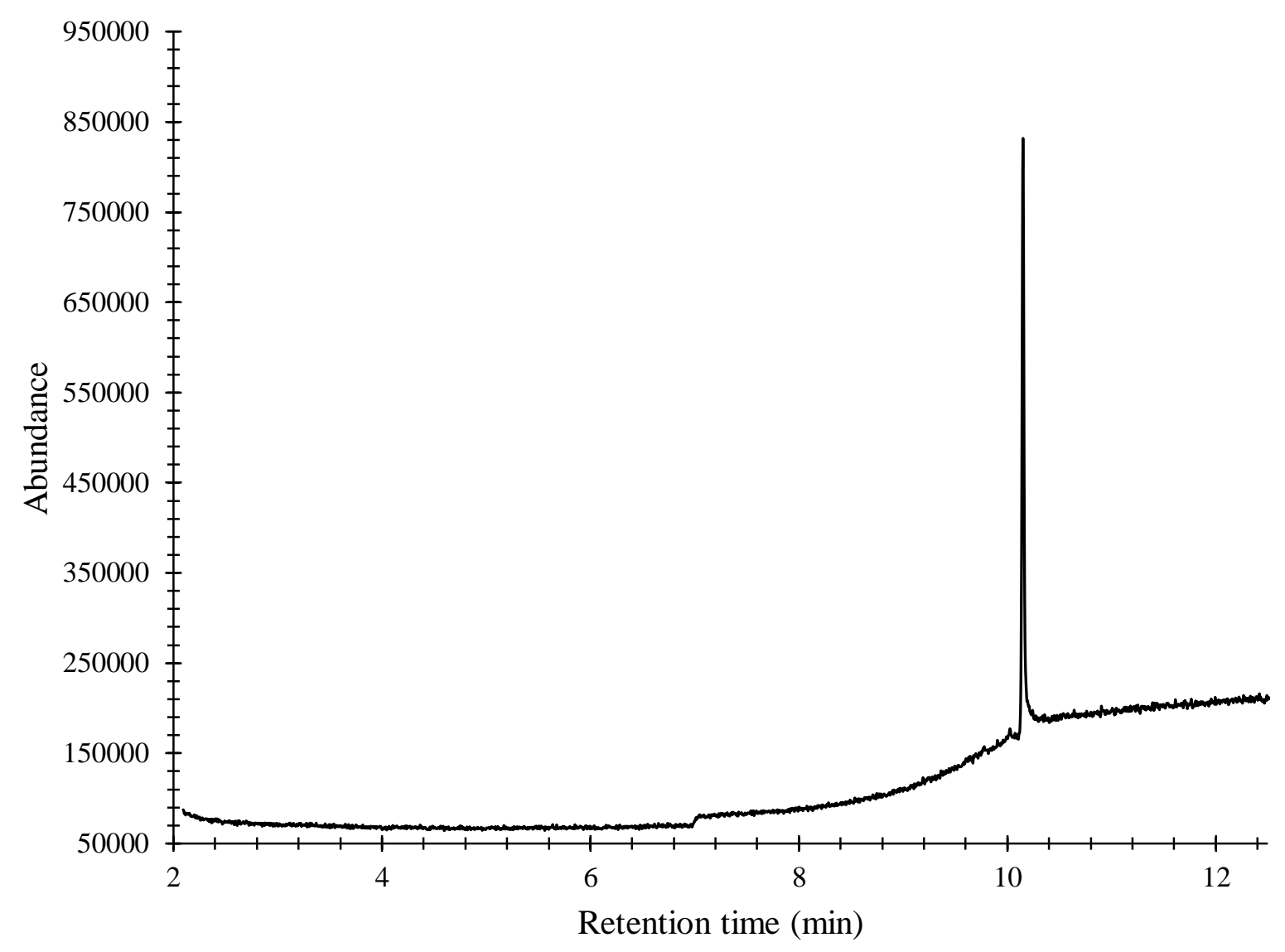

Figure 3.6. Total ion chromatogram of 125 ppm 25C-NBOMe meta isomer analyzed using the Agilent traditional GC-MS method. 
An example of a 1,250 ppm 25C-NBOMe para isomer chromatogram is shown in Figure 3.7. This analysis was completed using Agilent fast GC-MS, as indicated by the 6.2 minute total analysis time. The elution of the analyte occurred at 4.705 minutes and this was the only major peak seen in the chromatogram. Two minor peaks were seen prior to the elution of this isomer that were consistent with degradation products.

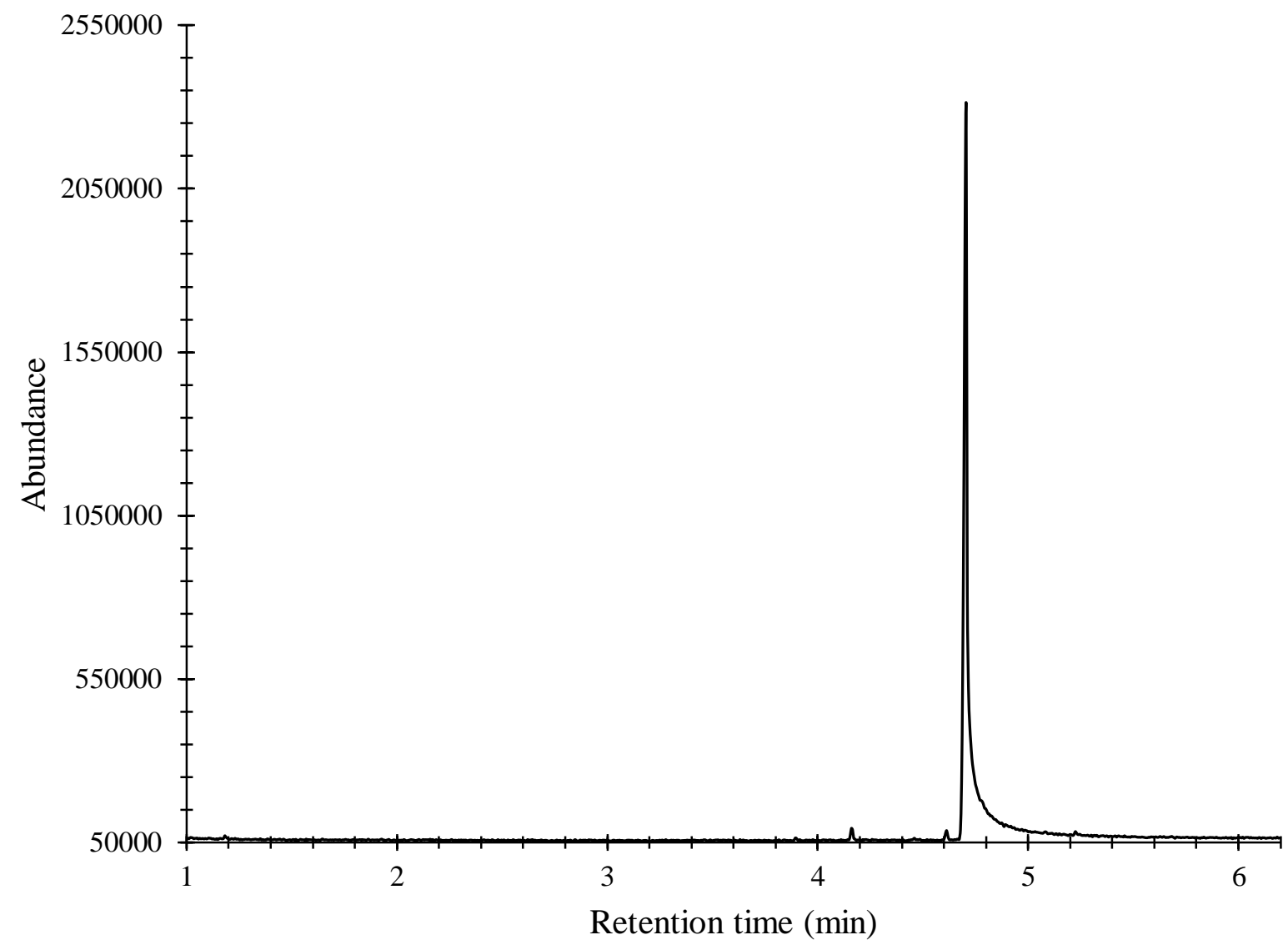

Figure 3.7. Total ion chromatogram of 1,250 ppm 25C-NBOMe para isomer analyzed using the Agilent fast GC-MS method. 
The chromatogram seen in Figure 3.8 is a 1,250 ppm 25I-NBOMe para isomer sample analyzed with Agilent traditional GC-MS. The elution time of this isomer was 11.773 minutes and it was the only major peak present in the chromatogram. One minor peak which was consistent with degradation products eluted prior to the analyte peak.

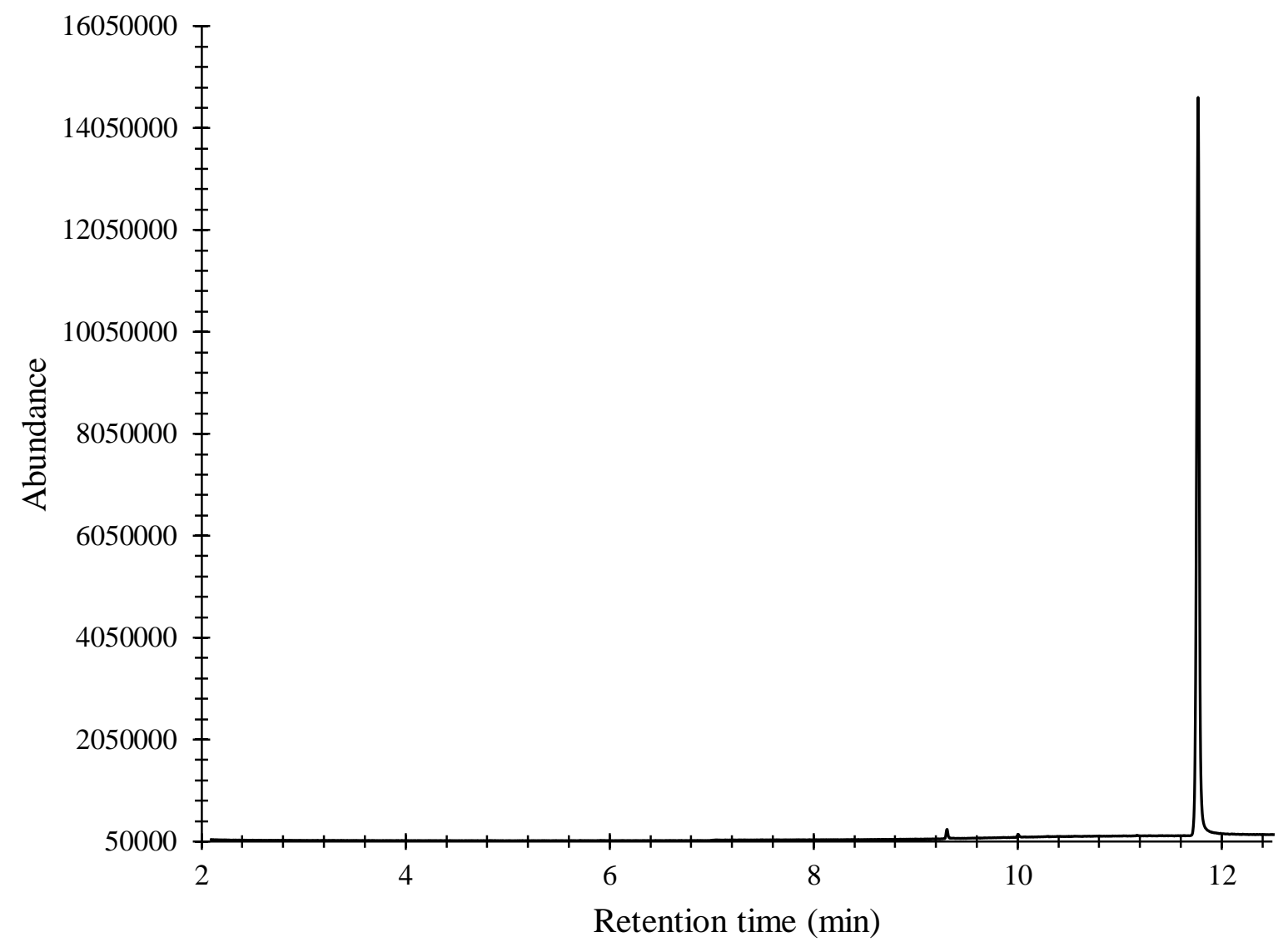

Figure 3.8. Total ion chromatogram of 1,250 ppm 25I-NBOMe para isomer analyzed using the Agilent traditional GC-MS method. 
Figure 3.9 is an Agilent fast GC-MS chromatogram of a $125 \mathrm{ppm}$ solution where all six NBOMe isomers were present. This is a demonstration of the most complex separation that occurred during this project as the elution of all six isomers occurred within a one-minute window. The elution occurred in the ortho, meta, para order for both drugs and the 25C-NBOMe isomers eluted before the 25I-NBOMe isomers. Several minor peaks were observed that were consistent with column or septum bleed and degradation products.

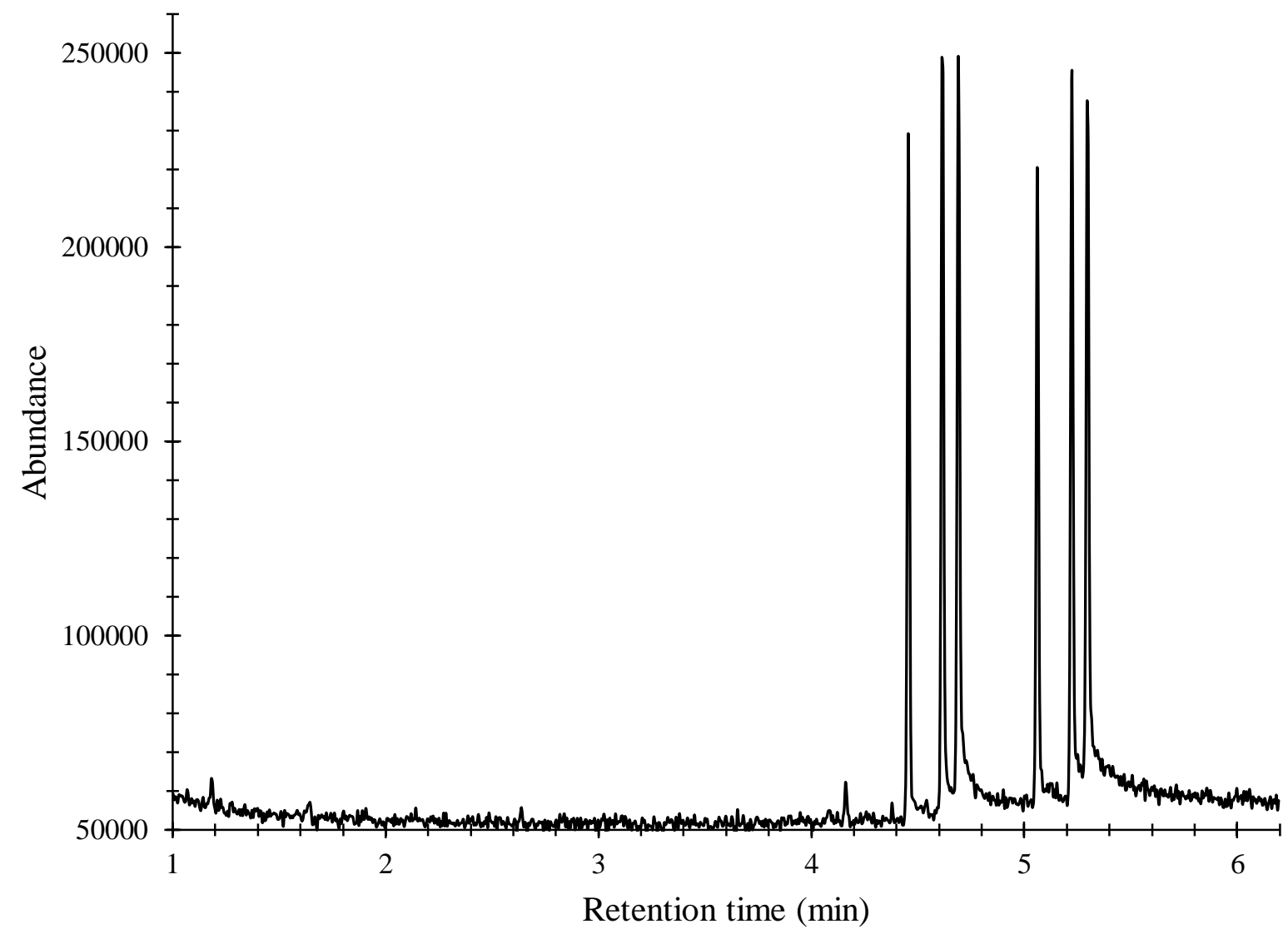

Figure 3.9. Total ion chromatogram of 125 ppm mixture of all six isomers using the Agilent fast GC-MS method. 
Figure 3.10 shows the chromatogram obtained from the same $125 \mathrm{ppm}$ solution containing all six isomers analyzed with Agilent traditional GC-MS. Once again, the elution occurred in the ortho, meta, para order with all of the 25C-NBOMe isomers eluting before the 25I-NBOMe isomers. The difference in peak height between the first three isomers (25C) and the last three isomers (25I) is partially caused by longitudinal diffusion; the $25 \mathrm{I}$ isomers spend more than a minute longer on the column than each $25 \mathrm{C}$ isomer. Several minor peaks were observed that were consistent with degradation products.

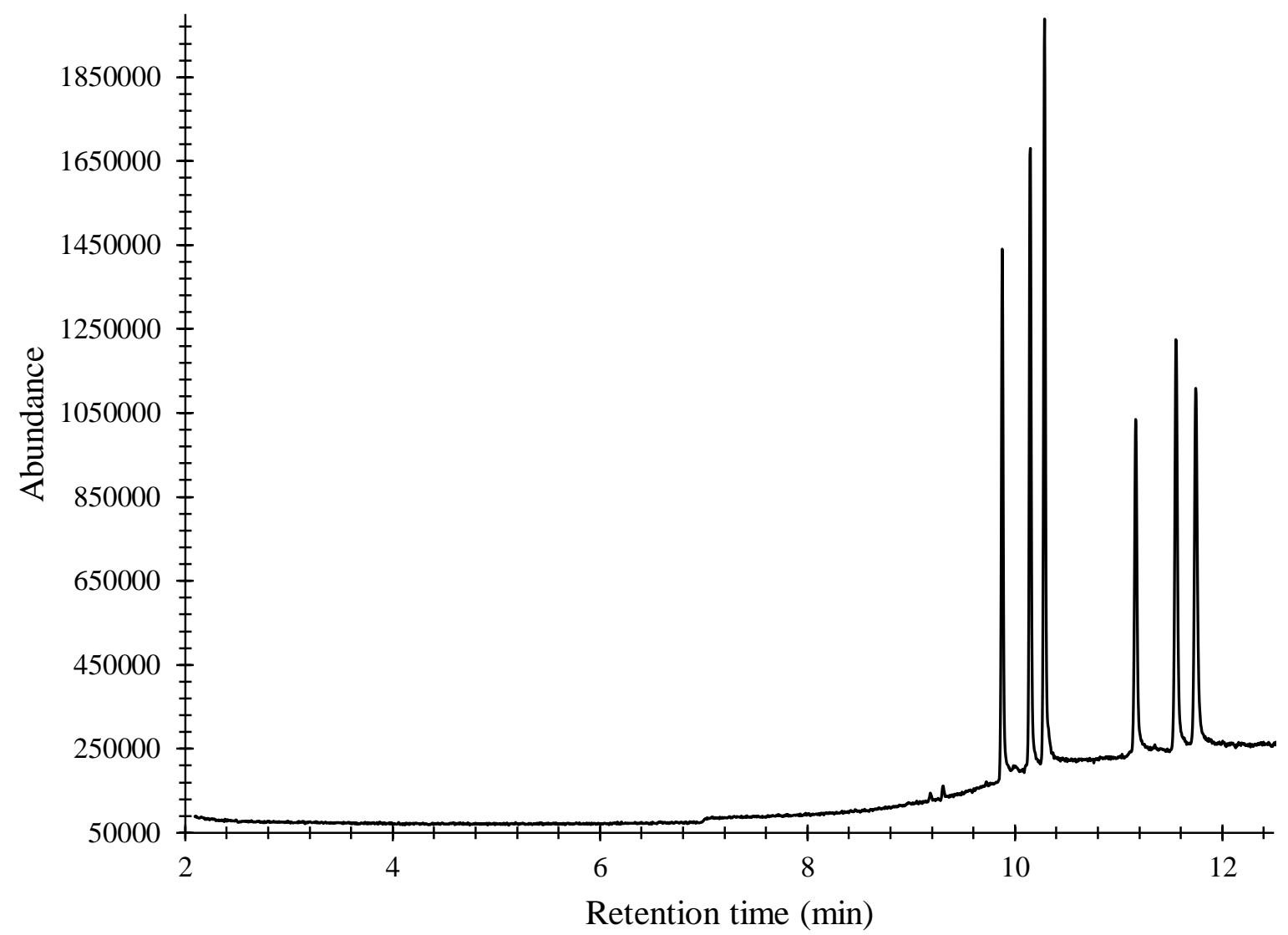

Figure 3.10. Total ion chromatogram of 125 ppm mixture of all six isomers using the Agilent traditional GC-MS method. 
An important aspect in the argument for the implementation of fast GC-MS into crime labs is the demonstration not only of the decrease in time for analysis, but also a demonstration that there is no negative impact to the chromatographic separation efficiency. To demonstrate this concept, a comparison of the GC resolutions and the number of theoretical plates was calculated for the Agilent fast GC-MS and Agilent traditional GC-MS samples using Equations 4 and 5. The main difference between these two analyses was the column with which the analyses were conducted. Equation 4 shows how the chromatographic resolution was used to evaluate the resolution of these two chromatograms, where $\mathrm{R}_{\mathrm{t}}$ indicates retention times of two adjacent isomers and $\mathrm{W}_{\mathrm{b}}$ indicates width of the peak at the base for peaks one and two [34].

$$
\mathrm{R}=2 *\left[\frac{\left(\mathrm{R}_{\mathrm{t}_{2}}-\mathrm{R}_{\mathrm{t}_{1}}\right)}{\left(\mathrm{W}_{\mathrm{b}_{1}}+\mathrm{W}_{\mathrm{b}_{2}}\right)}\right]
$$

The equation to determine the number of theoretical plates is shown in Equation 5, where $\mathrm{N}$ is the number of theoretical plates, $\mathrm{R}_{\mathrm{t}}$ is the retention time of the peak of interest, and $\mathrm{W}_{\mathrm{h}}$ represents the width at half maximum of the peak of interest [34]. The larger the value of $\mathrm{N}$ value, the more theoretical plates, and the better the separation that can be achieved.

$$
\mathrm{N}=5.545\left(\frac{\mathrm{R}_{\mathrm{t}}}{\mathrm{W}_{\mathrm{h}}}\right)^{2}
$$


Table 3.1 shows the results for the Agilent fast GC-MS and Agilent traditional GC-MS separation of the $125 \mathrm{ppm}$ mixture of all six NBOMe isomers. This is an example of what a single analysis of the separation efficiency between these two techniques looks like, where $\mathrm{R}_{\mathrm{t}}$ is the retention time, $\mathrm{W}_{\mathrm{b}}$ is the width at the base of the peak, and $\mathrm{W}_{\mathrm{h}}$ is the width at the half maximum of the peak.

Table 3.1. Agilent Fast GC-MS vs Agilent traditional GC-MS separation efficiency results.

\begin{tabular}{|l|c|c|c|c|c|c|c|c|}
\hline \multirow{2}{*}{ Component } & \multicolumn{4}{|c|}{ Agilent Fast GC-MS } & \multicolumn{4}{c|}{ Agilent Traditional GC-MS } \\
\cline { 2 - 9 } & $\mathbf{2 5 C - O}$ & $\mathbf{2 5 C}-\mathbf{M}$ & $\mathbf{2 5 I}-\mathbf{O}$ & $\mathbf{2 5 I}-\mathbf{M}$ & $\mathbf{2 5 C}-\mathbf{O}$ & $\mathbf{2 5 C}-\mathbf{M}$ & $\mathbf{2 5 I}-\mathbf{O}$ & $\mathbf{2 5 I}-\mathbf{M}$ \\
\hline $\mathrm{R}_{\mathrm{t} 2}$ & 4.618 & 4.693 & 5.226 & 5.299 & 10.15 & 10.288 & 11.557 & 11.747 \\
\hline $\mathrm{R}_{\mathrm{t} 1}$ & 4.458 & 4.618 & 5.064 & 5.226 & 9.881 & 10.15 & 11.168 & 11.557 \\
\hline $\mathrm{W}_{\mathrm{b} 1}$ & 0.096 & 0.093 & 0.074 & 0.092 & 0.222 & 0.156 & 0.269 & 0.198 \\
\hline $\mathrm{W}_{\mathrm{b} 2}$ & 0.093 & 0.106 & 0.092 & 0.063 & 0.156 & 0.176 & 0.198 & 0.229 \\
\hline Resolution & 1.69 & 0.754 & 1.95 & 0.942 & 1.42 & 0.831 & 1.67 & 0.890 \\
\hline & & & & & & & & \\
\hline $\mathrm{R}_{\mathrm{t}}$ & 4.458 & 4.618 & 5.064 & 5.226 & 9.881 & 10.15 & 11.168 & 11.557 \\
\hline $\mathrm{W}_{\mathrm{h}}$ & 0.016 & 0.016 & 0.015 & 0.015 & 0.019 & 0.023 & 0.032 & 0.032 \\
\hline $\begin{array}{l}\text { Theoretical } \\
\text { plates }\end{array}$ & $4.3 \times 10^{5}$ & $4.6 \times 10^{5}$ & $6.3 \times 10^{5}$ & $6.7 \times 10^{5}$ & $1.5 \times 10^{6}$ & $1.1 \times 10^{6}$ & $6.8 \times 10^{5}$ & $7.2 \times 10^{5}$ \\
\hline
\end{tabular}


Table 3.2 shows the averaged results for the Agilent fast GC-MS and Agilent traditional GC-MS separation of the $125 \mathrm{ppm}$ mixture of all six NBOMe isomers. The average was taken for the resolution and number of theoretical plates for each isomer and technique. P-values are also provided to indicate the differences among sample means. The results indicate that the average resolutions between the Agilent fast GC-MS and the Agilent traditional GC-MS are not significantly different. The results for the average number of theoretical plates calculated using all six isomers are not significantly different. However, when each subset of positional isomers was used to determine the average number of theoretical plates, there was a significant difference observed.

Table 3.2. Averaged results for Agilent fast GC-MS vs Agilent traditional GC-MS separation efficiency.

\begin{tabular}{|l|c|c|}
\hline \multicolumn{1}{|c|}{ Component } & Agilent Fast GC-MS & Agilent Traditional GC-MS \\
\hline Average resolution & 1.379 & 1.912 \\
\hline RSD (\%) & 18.53 & 19.32 \\
\hline t-test: $25 \mathrm{C}$ & \multicolumn{2}{|c|}{0.655} \\
\hline t-test: $25 \mathrm{I}$ & \multicolumn{2}{|c|}{0.478} \\
\hline t-test: All & \multicolumn{2}{|c|}{0.476} \\
\hline Average theoretical plates & 616,119 & 896,470 \\
\hline RSD (\%) & 22.47 & 21.89 \\
\hline t-test: $25 \mathrm{C}$ & \multicolumn{2}{|c|}{0.017} \\
\hline t-test: $25 \mathrm{I}$ & \multicolumn{2}{|c|}{0.008} \\
\hline t-test: All & \multicolumn{2}{|c|}{0.372} \\
\hline
\end{tabular}


Figure 3.11 is a 12.5 ppm sample analyzed with Agilent fast GC-MS containing the ortho, meta, and para isomers of 25C-NBOMe.

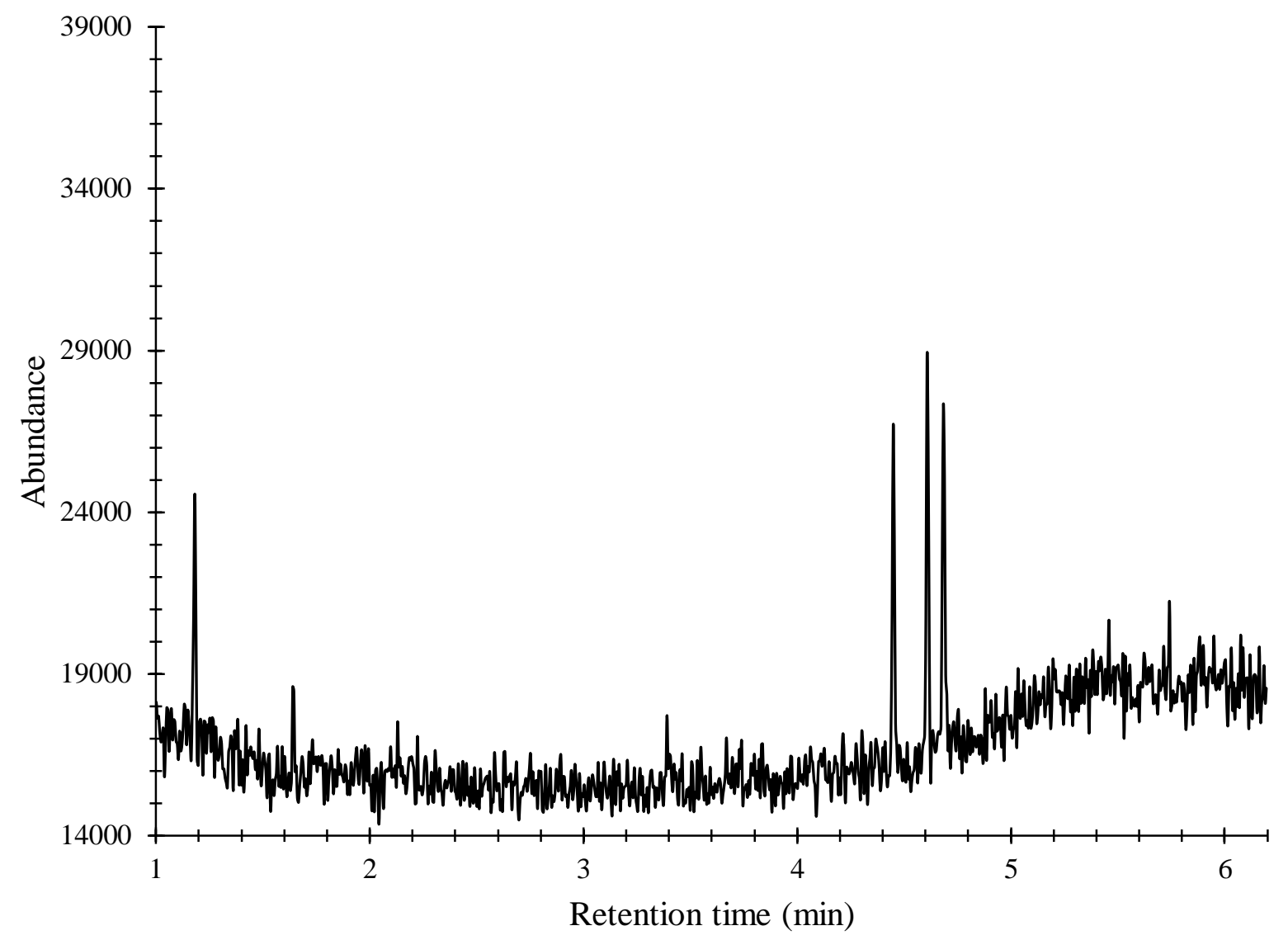

Figure 3.11. Total ion chromatogram of $12.5 \mathrm{ppm}$ mixture of the 25C-NBOMe mixture using the Agilent fast GC-MS method. This sample was 4 weeks old and showed contamination peaks of PDMS from the storage vial caps.

A relatively intense peak eluted around 1.20 minutes. This peak was identified as the septum bleed seen throughout this experiment. However, this example occurred toward the end of the Agilent fast GC-MS portion of this experiment and provides a demonstration of a phenomenon that occurred during this project. The background contamination peaks increased in intensity over time to the point where they were almost equivalent in height with the $12.5 \mathrm{ppm}$ NBOMes by the end of the four weeks of Agilent fast GC-MS analysis. The correlation between 
time and PDMS background peaks indicates that the PDMS contamination originates upstream of the injection port. Our GC vials used PTFE silicon vial caps for an extended period of time, which, according to literature, may have contributed to the rapid increase in contaminants present in the samples [35].

Another example of increased contamination over time is shown below in Figure 3.12, which displays a 12.5 ppm 25I-NBOMe mixture of all three positional isomers analyzed with Agilent traditional GC-MS. Due to the smaller split ratio, the effects of the contamination are not as apparent compared to the analyte peaks. However, the contamination present in this solution increased over time as well, consistent with vial cap septum contamination.

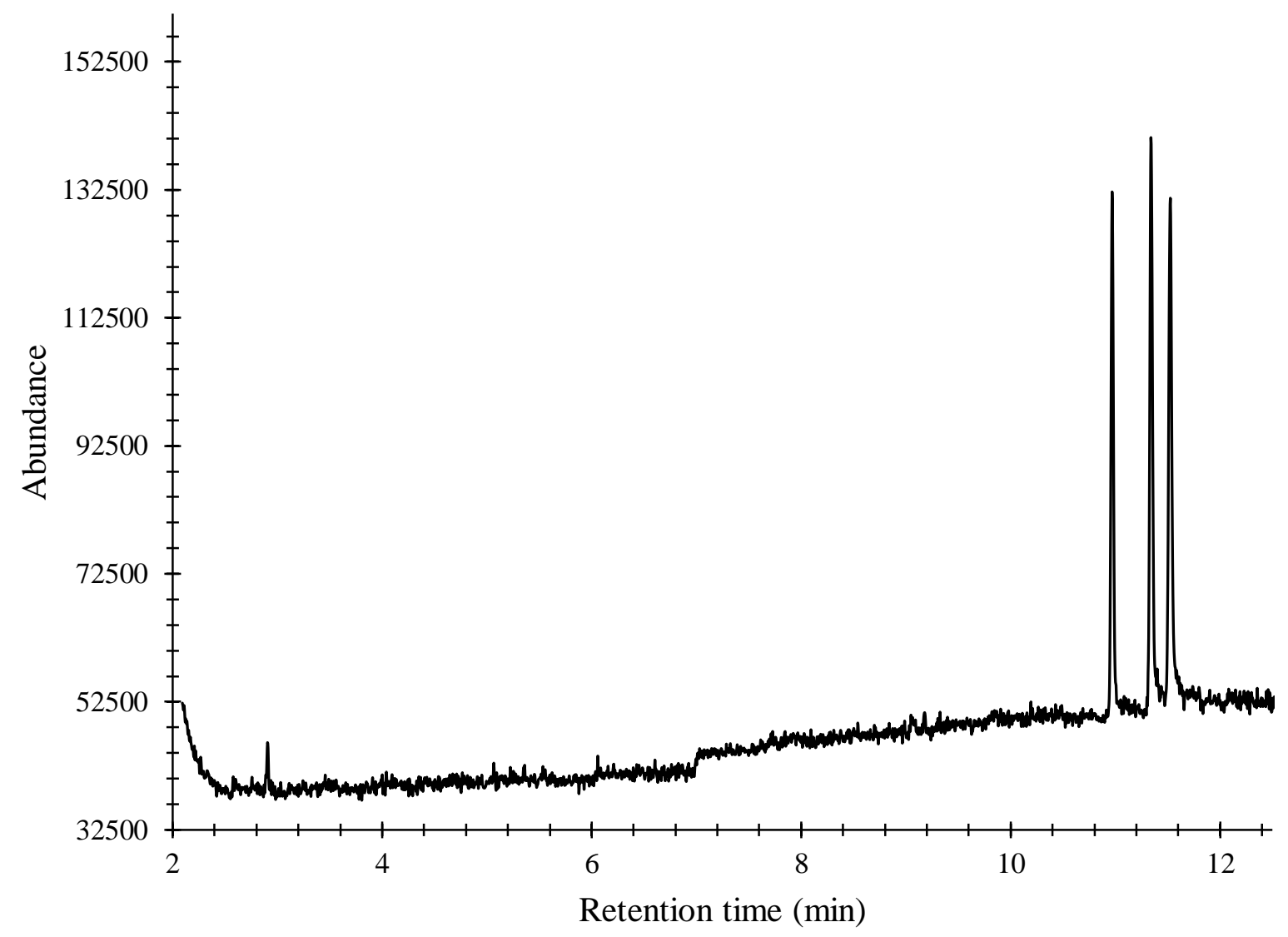

Figure 3.12. Total ion chromatogram of 12.5 ppm mixture of the 25I-NBOMe mixture using the Agilent traditional GC-MS method. This sample was $\sim 8$ weeks old and showed contamination peaks of PDMS from the storage vial caps. 
Several trends were observed from these exemplar chromatograms. The first trend was that the elution of each NBOMe was always in the order of ortho, meta, para. Second, the 25C-NBOMe isomers had a shorter retention time than the 25I-NBOMe isomers, in agreement with the results of Casale and Hays of the DEA [2]. Third, when the NBOMe samples were stored for prolonged periods (e.g. weeks), the background levels increased significantly. However, the PDMS peaks eluted far away from the analytes of interest, so no interference with the analysis occurred.

\subsection{GC SPSS Interpretation}

SPSS software was used to evaluate the significance of different factors on the withinfactor to between-factor variance of the retention time, retention index, and peak area data. The factors assessed included the positional isomer, concentration, the day of analysis, the week of analysis, the instrument, the tune profile, and the speed of GC. The assumptions made by this technique are that there is homogeneity of variance, that the populations are normally distributed, and that each value is sampled independently [28]. 
To verify these assumptions were met the homogeneity of variance and a Q-Q plot to indicate normality were generated for each factors and variable combination. An exemplar Q-Q plot can be seen in Figure 3.13. The Q-Q plot represents the normality of the retention indices data for the $12.5 \mathrm{ppm}$ 25C-NBOMe ortho data collected via the PerkinElmer traditional analysis. The retention indices correlate with the expected normal value, indicating that the data was normally distributed.

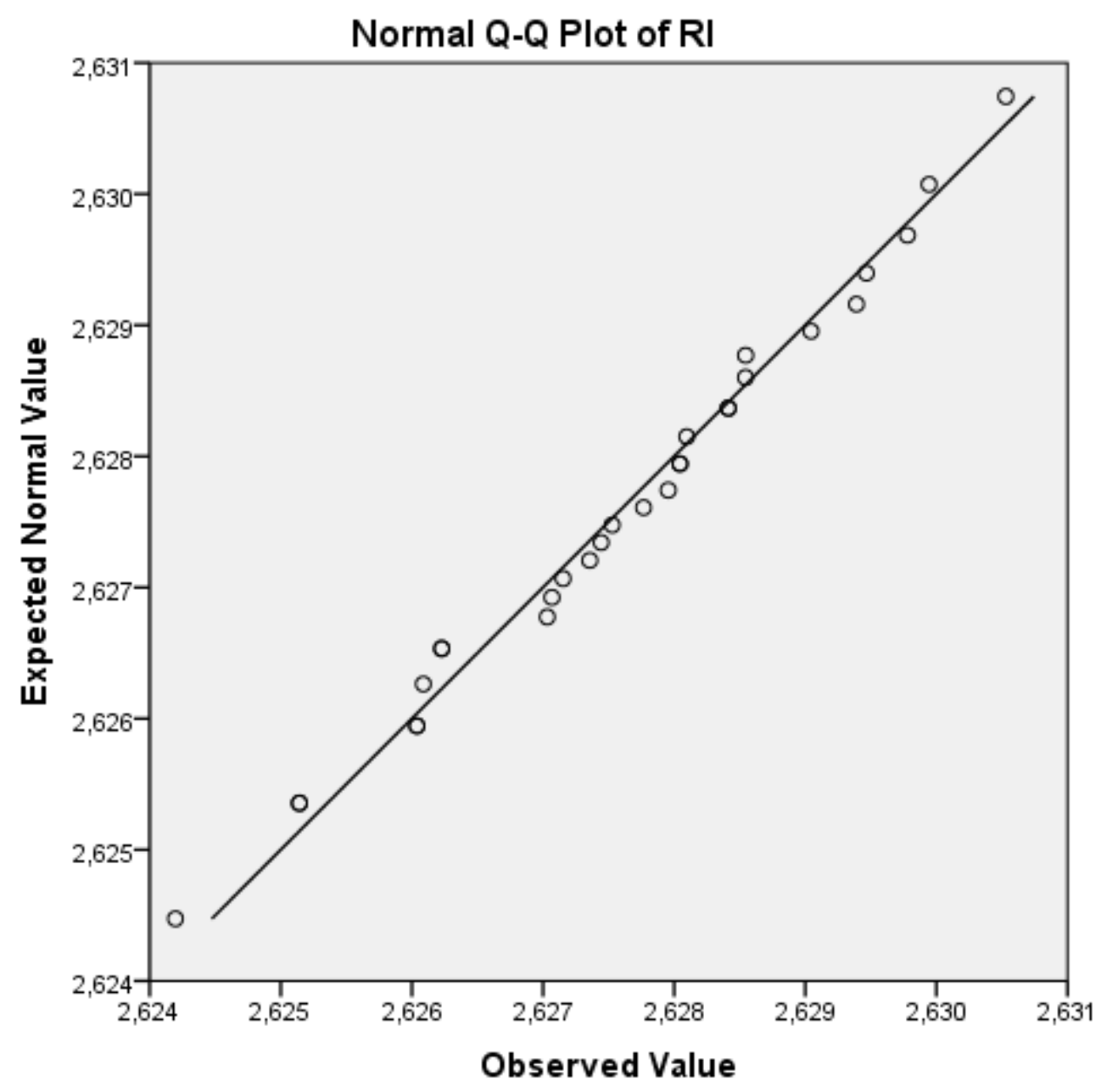

Figure 3.13. $Q-Q$ plot of 12.5 ppm 25C-NBOMe ortho isomer demonstrating normality of retention time data. 
The observed variance in the retention times, retention indices, and peak areas were analyzed to determine which sources contributed significantly to the variation. The results for the ANOVA analysis of the retention time, retention indices, and peak area can be seen below in Table 3.3. The results are provided as p-values that were typically compared to the alpha value ( $\alpha$ ) of 0.05 (95\% confidence interval).

Table 3.3. ANOVA Retention time and retention index at the $95 \%$ confidence level.

\begin{tabular}{|l|c|c|c|}
\hline \multicolumn{4}{|c|}{ Significance (p-value) } \\
\hline \multicolumn{1}{|c|}{ Factor } & Retention time & Retention indices & Peak area \\
\hline Isomer & $<1 \times 10^{-5}$ & $<1 \times 10^{-5}$ & 0.23 \\
\hline Concentration & 0.85 & 0.61 & $<1 \times 10^{-5}$ \\
\hline Tune & 0.99 & 1.0 & 0.12 \\
\hline Speed of GC & $<1 \times 10^{-5}$ & 0.91 & $<1 \times 10^{-5}$ \\
\hline *Instrument & $<1 \times 10^{-5}$ & $8.5 \times 10^{-4}$ & $<1 \times 10^{-5}$ \\
\hline Week & $<1 \times 10^{-5}$ & 0.99 & $1.2 \times 10^{-3}$ \\
\hline Day & 1.0 & 1.0 & 0.84 \\
\hline
\end{tabular}

*When retention indices are analyzed by concentration, there is no significant difference between instruments.

The null hypothesis for each of these analyses is that the population means between variables are equal; i.e. each isomer has the same mean retention time [36]. If the significance, represented here by the p-value, is less than the $0.05 \alpha$ value, then the null hypothesis is rejected because at least one population mean is statistically different at the $95 \%$ confidence level [37]. This interpretation was then applied to each of the factors analyzed during this project to determine if the factor contributed significantly at the $95 \%$ confidence level to the variation in the retention time, retention indices, or peak area.

The results indicate that the isomer contributes significantly to the variation in the retention time and retention indices, but not significant at the $95 \%$ confidence level to the peak area. On the other hand, the concentration of the solution contributes significantly to the peak area, whereas it does not significantly affect the variation in the retention time or retention index. 
As anticipated, the effect of the tune profile does not play a significant role on any of the GC-related variables. The speed of GC had a significant effect on the variation in the retention time and peak areas, which was expected because the split ratio was varied in each method. The different split ratio contributed to the differences in peak area and the retention time varied based on the column used for each analysis.

The speed of GC did not have a statistically significant effect on the retention indices because these values were normalized to an n-alkane ladder. However, the instrument did have a statistically significant contribution to the variation in the retention time, retention indices, and peak area. The two instruments had different columns and manufacturers, which could contribute to the variation in retention times due to different functional groups and chemical properties. The retention time and peak area results were explainable, as described previously, but the retention indices results were unexpected. However, when the retention indices were analyzed by concentration, there was no significant difference at the $95 \%$ confidence level.

The week of analysis had a significant effect on the variance of the retention times and peak areas, but not the retention indices. This result can be explained because the retention time will decrease over time as the stationary phase evaporates from the column [38]. Also, the concentration may slowly increase over time due to solvent evaporation. The effect of the day of analysis was not significant to any of these variables as the changes from day-to-day were minimal in comparison to the changes between weeks. 
Another statistical assessment known as the eta squared test was used to determine the proportion of the total variance in a dependent variable that was associated with variation in an independent variable [39]. Table 3.4 below contains the eta squared results.

Table 3.4. Eta squared retention time, retention index, and peak area results.

\begin{tabular}{|l|c|c|c|}
\hline \multicolumn{3}{|c|}{ Eta squared } \\
\hline \multicolumn{1}{|c|}{ Factor } & Retention time & Retention index & Peak area \\
\hline Isomer & 0.039 & 0.99 & $4.5 \times 10^{-3}$ \\
\hline Concentration & $2.1 \times 10^{-4}$ & $1.2 \times 10^{-3}$ & 0.52 \\
\hline Tune & $1.4 \times 10^{-3}$ & $3.0 \times 10^{-4}$ & $1.3 \times 10^{-2}$ \\
\hline Speed of GC & 0.93 & $1.7 \times 10^{-3}$ & $7.1 \times 10^{-2}$ \\
\hline Instrument & 0.96 & $9.0 \times 10^{-3}$ & $8.6 \times 10^{-2}$ \\
\hline Week & 0.23 & $1.5 \times 10^{-3}$ & $1.6 \times 10^{-2}$ \\
\hline Day & $1.1 \times 10^{-3}$ & $5.7 \times 10^{-4}$ & $5.2 \times 10^{-3}$ \\
\hline
\end{tabular}

The interpretation for the eta squared results was that output value multiplied by 100 indicates the percentage of variance being explained in the dependent variable by variation in the factor being analyzed. For example, the isomer factor explains $99 \%$ of the variance in retention indices, but only $3.9 \%$ of the retention time. This result is an indication that there are other factors outside the system analyzed that impact the retention time results, the main factor being the speed of the GC.

The concentration of analyte contributes significantly to the variance associated with peak area at approximately $52 \%$, whereas the tune profile explains little to no variation in the retention time, retention indices, and peak area. The speed of GC and instrument factors contribute significantly to the variation in the retention time, but play much less of a role on the retention indices because the indices have been normalized to an n-alkane ladder. The speed of GC and instrument factors correlate strongly, which explains why the sum of the eta squared values in the retention time column of Table 3.4 exceeds 1. 
The results for the day of analysis and week of analysis were consistent with the ANOVA results. The week of analysis contributes more to the variation of retention time than the day of analysis and both factors contribute only minutely to the variation in the retention indices and peak area variables.

\subsection{GC Results}

Figure 3.14 shows three box and whisker plots of the retention indices broken down by each methodology studied during this research. The boxes show the interquartile range of the data and the whiskers show the upper and lower levels for the non-rejected data points. 

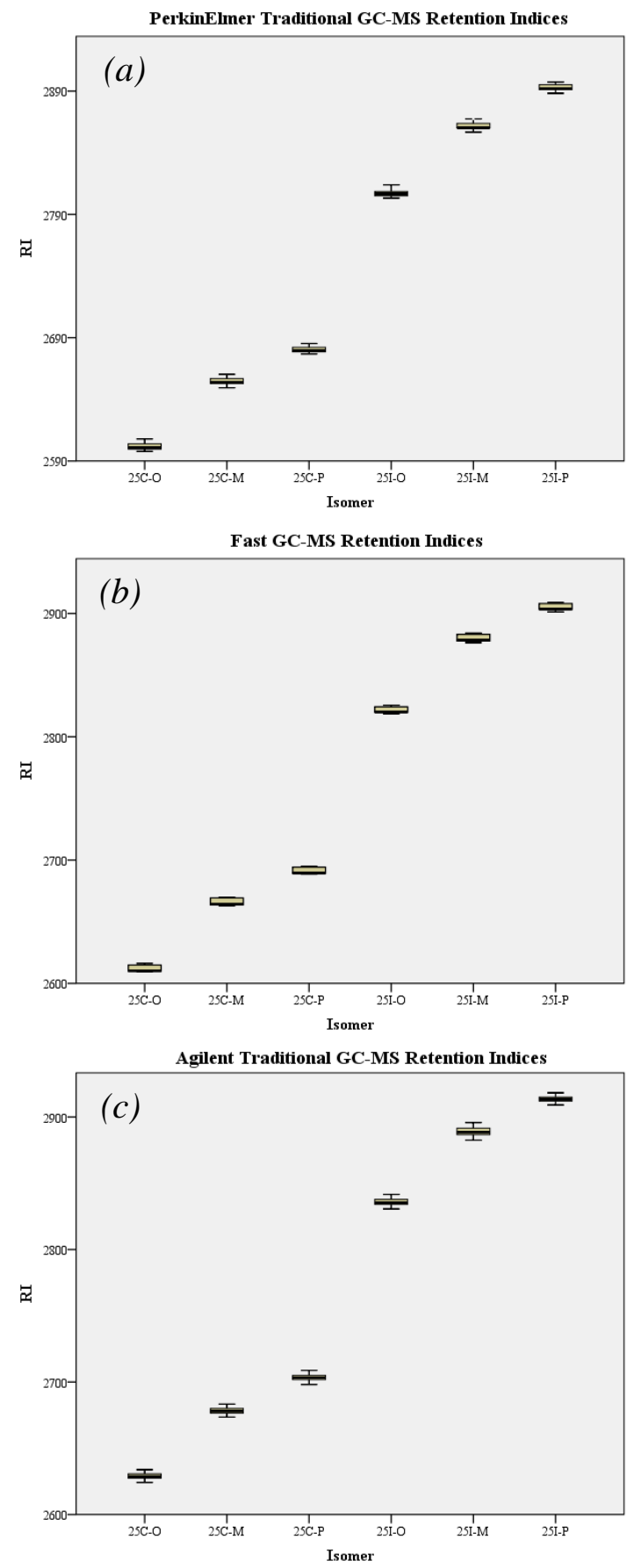

Figure 3.14. Box and whisker plots for the retention indices corresponding to the PerkinElmer traditional GC-MS (a), Agilent fast GC-MS (b), and the Agilent traditional GC-MS (c). 
Figure 3.15 shows the retention indices broken down by each methodology and fixed concentration studied during this research. The range of values is so small per fixed concentration range that it is difficult to even identify the box and whisker plots. 

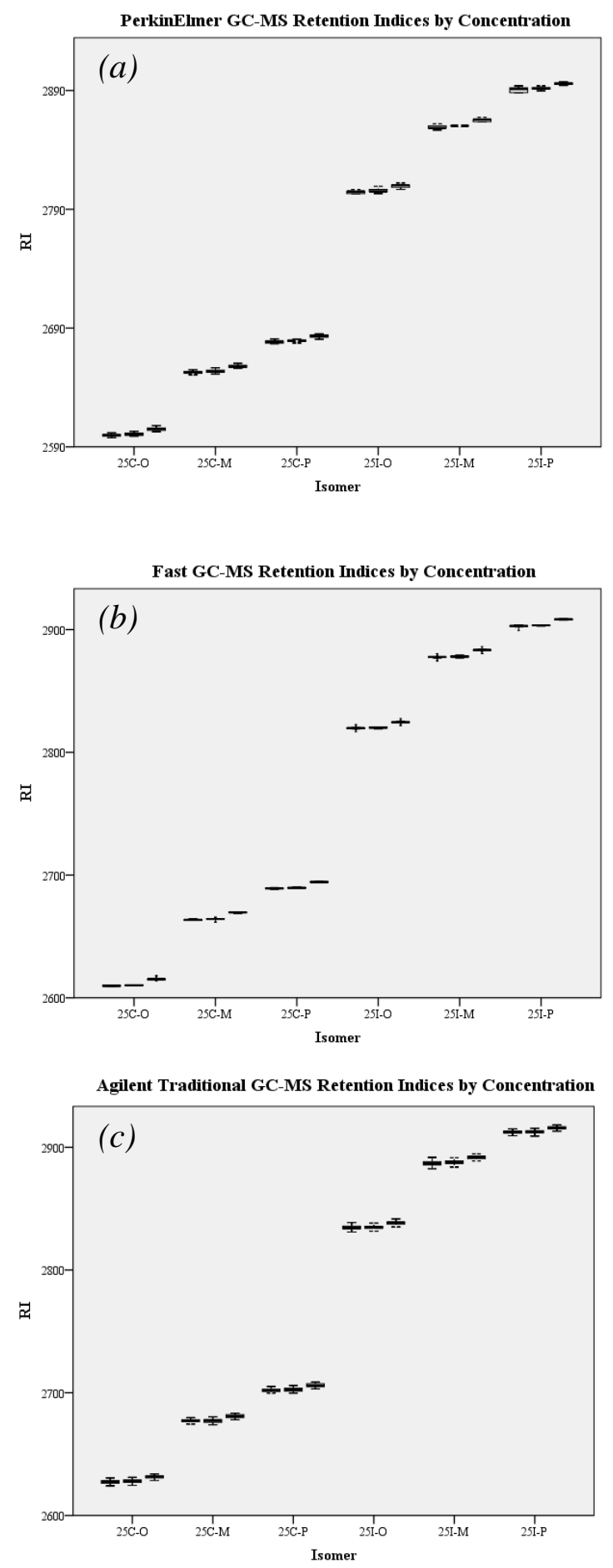

Figure 3.15. Box and whisker plots for the retention indices broken down by the methodology and the fixed concentration of the sample corresponding to the PerkinElmer traditional GC-MS (a), Agilent fast GC-MS (b), and the Agilent traditional GC-MS (c). The three boxes at each positional isomer are for the 12.5, 125, and 1250 ppm solutions, respectively. 
Figure 3.16 shows the results for the combined retention indices across all methodologies studied by this research. Even with the increased uncertainty, because of the different retention times, the difference between isomers is clear.

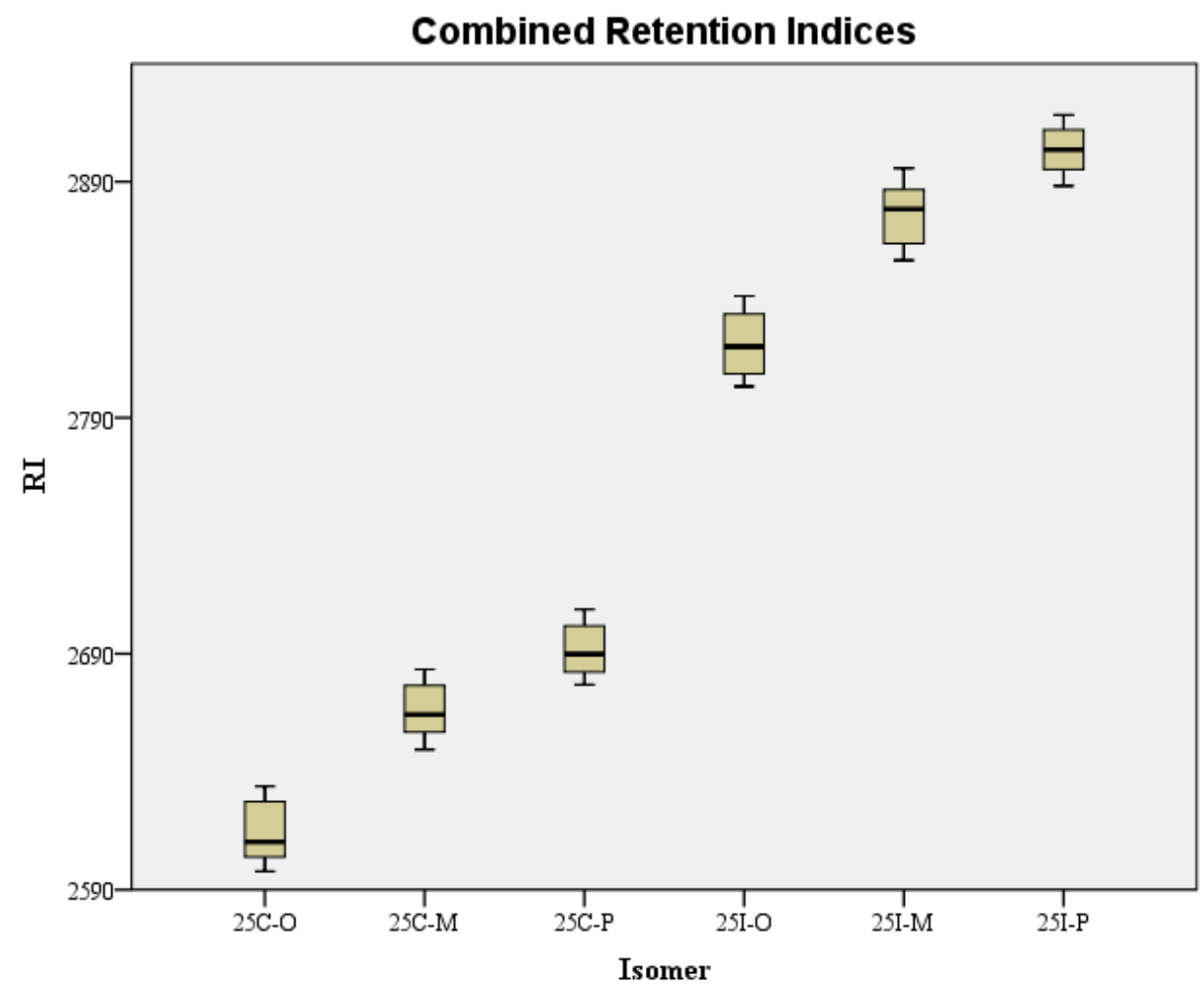

Figure 3.16. Combined (across three concentrations) retention indices results for all methodologies studied. 
Table 3.5 contains the results for the $95 \%$ confidence intervals for the retention indices when all three techniques and concentrations were combined. These results indicate that even when accounting for differences between methodologies and instrumentation, four of the six $95 \%$ confidence interval ranges were distinct from one another at the $95 \%$ confidence interval.

Table 3.5. $95 \%$ confidence intervals for the retention indices of the combined data.

\begin{tabular}{|l|c|}
\hline \multicolumn{1}{|c|}{ Isomer } & 95\% C.I. for RI \\
\hline 25C-NBOMe ortho & $2614 \pm 15$ \\
\hline 25C-NBOMe meta & $* 2666 \pm 13$ \\
\hline 25C-NBOMe para & $2692 \pm 13$ \\
\hline 25I-NBOMe ortho & $2821 \pm 16$ \\
\hline 25I-NBOMe meta & $* 2877 \pm 15$ \\
\hline 25I-NBOMe para & $2904 \pm 12$ \\
\hline
\end{tabular}

* Indicates not statistically different at the $95 \%$ confidence interval

Due to the much larger inter-isomer to intra-isomer variability, it was quite easy to group the retention indices by isomer alone. However, the $95 \%$ confidence intervals for each concentration were so tightly grouped that isomers were not grouped between concentrations. Instead of the $95 \%$ confidence interval, the data is best visualized as shown previously in Figure 3.15 as a range around the median value, which allows for visualization of the isomer groupings by concentration. 
Figure 3.17 shows the combined retention indices results for all methodologies studied during this research broken down by the instrument used to analyze the sample. The labels correspond to each methodology (PerkinElmer, Fast, and Agilent) and every set of three box plots corresponds to an NBOMe isomer (25C-O to 25I-P).

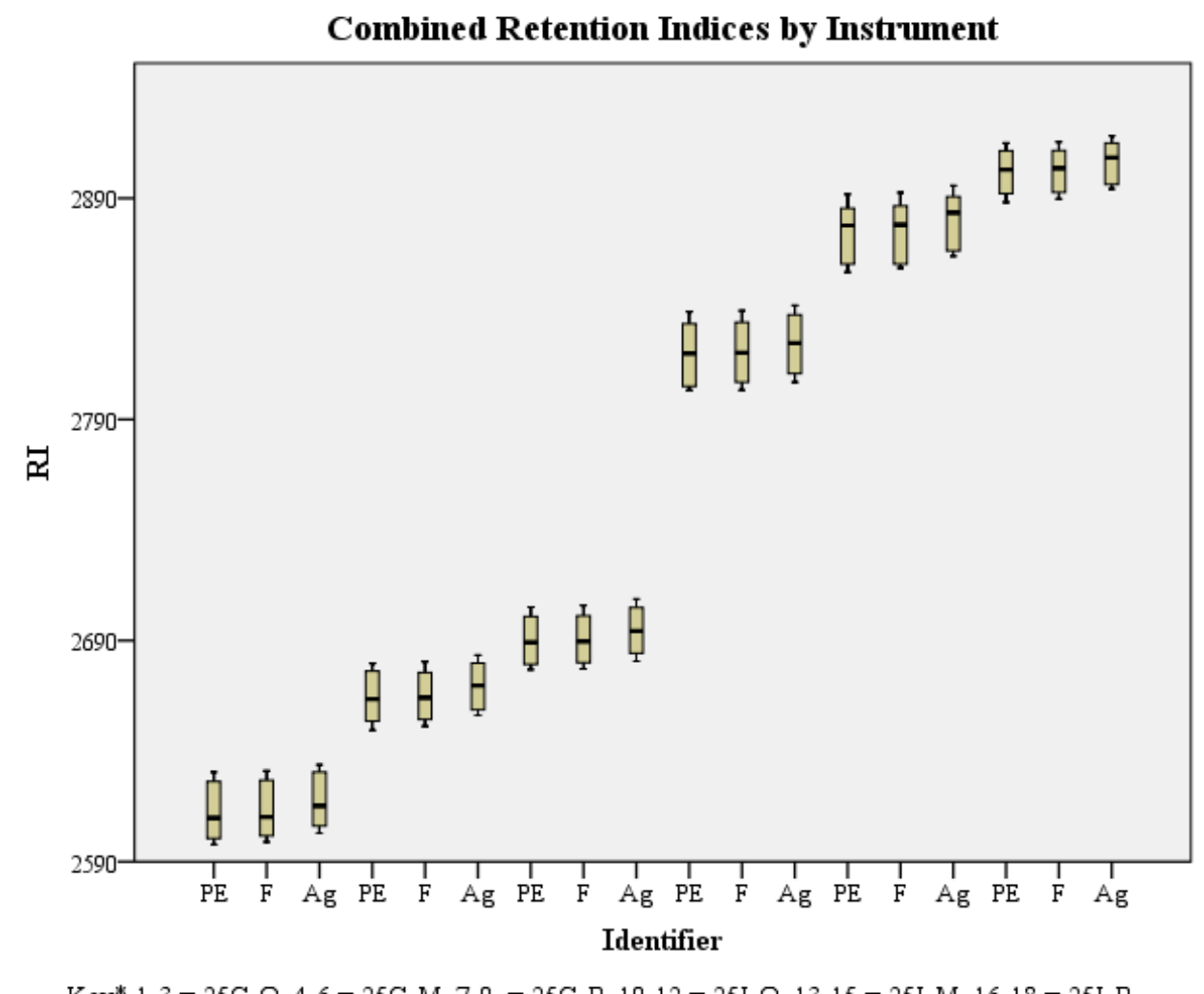

Figure 3.17. Combined retention indices results broken down instrumental methodology. Each set of three box and whisker plots corresponds to the PerkinElmer, Agilent fast, and Agilent traditional retention indices results per isomer. 
Table 3.6 shows the results for the relative percent standard deviation (\% RSD) and the expanded uncertainty $(2 \sigma)$ for the retention times of each technique. The $\%$ RSD and expanded uncertainty were chosen as measures of retention time variability per technique to indicate which technique had the most reproducible results over the course of this project. The reproducibility of the retention times based on \% RSD and expanded uncertainty was best for the Agilent fast GCMS analysis and worst for the Agilent traditional analysis. Table 3.6 shows that the Agilent traditional analysis was separated into two categories which correspond to the data collected before and after a leak around the transfer line was fixed. The pooled standard deviation was used to determine the \% RSD and expanded uncertainty, which contributed to the large increase in \% RSD and expanded uncertainty as compared to the other techniques. There are several different approaches to the assessment of retention time reproducibility. Two common approaches are the use of $\mathrm{a} \pm 2 \% \mathrm{RSD}$ or a \pm 0.1 minute retention time difference [40-43]. The Agilent fast GC-MS and PerkinElmer traditional analyses performed better than both of these criteria, while the Agilent traditional analysis performed better than the $\pm 2 \%$ RSD, but slightly worse than the \pm 0.1 minute retention time difference criteria.

Table 3.6. $R S D$ results for the retention time reproducibility.

\begin{tabular}{|c|c|c|}
\hline Technique & $\operatorname{RSD}(\%)$ & 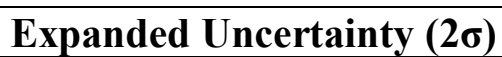 \\
\hline PerkinElmer & 0.096 & 0.020 \\
\hline Agilent fast analysis & 0.028 & 0.003 \\
\hline$*$ Agilent analysis $1-7$ & \multirow{2}{*}{1.26} & \multirow{2}{*}{0.27} \\
\hline *Agilent analysis $8-15$ & & \\
\hline
\end{tabular}

*Agilent traditional analyses broken down into two parts and the pooled standard deviation was used to determine the \%RSD and expanded uncertainty due to air leak adjustment.

Several conclusions can be drawn from the GC results. The first is that the retention indices grouped by technique were very precise and all isomers can be differentiated on any technique. When analyzed at the concentration level, the very small intra-concentration 
variability, as compared to the inter-isomer variability, is observed. The grouping of the retention indices between techniques increased the range of values, but still the inter-isomer variation was much greater than the intra-isomer variation. Finally, the \% RSD results for the retention time reproducibility indicate that all three techniques were better than the $\pm 2 \% \mathrm{RSD}$ and all but the Agilent traditional analysis was better than the \pm 0.1 minute retention time difference criteria currently used by many organizations. The significance of these results is that the intra-isomer variability for retention times and retention indices is much smaller than the inter-isomer variability allowing for ease of identification of these isomers within crime labs. Also, the reproducibility results indicate that the $\pm 2 \% \mathrm{RSD}$ or \pm 0.1 minute retention time difference match criteria recommended by many organizations is less stringent than the reproducibility seen in this study.

Based on the average chromatographic resolution, the average number of theoretical plates, \% RSD values, and actual length of analysis, it is concluded that the Agilent fast GC-MS performed as well as, if not better than, the Agilent traditional GC-MS analyses performed in this research. The value of this information is that crime labs could use fast GC-MS instead of traditional GC-MS and thereby take advantage of the faster analysis times to increase laboratory productivity. Concerns that have been raised by the crime lab community in relation to peak resolution, reproducibility, ease of conversion, and increased maintenance were addressed in this project as the Agilent fast GC-MS performance was on par or better than that of the Agilent traditional GC-MS for all of these categories and no additional downtime outside of routine instrument maintenance was observed. 


\subsection{Mass Spectra Results}

Figure 3.18 shows the $12.5 \mathrm{ppm}$ results for the three 25C-NBOMe isomers analyzed with Agilent fast GC-MS. The base peak is at $m / z 121$ and no molecular ion peak is present in the spectra. The ions at $\mathrm{m} / \mathrm{z} 150$ and 91 are the second and third most abundant ions. The spectra are truncated from $\mathrm{m} / \mathrm{z} 50$ to $\mathrm{m} / \mathrm{z} 350$ to eliminate the majority of the contamination peaks. One contamination peak that is still present is at $\mathrm{m} / \mathrm{z} 207$, which is due to column or septum bleed. The relative abundance of the $\mathrm{m} / z, 150$ and $\mathrm{m} / \mathrm{z} 91$ peaks are of particular importance as these two ions can help distinguish the positional isomers of 25C-NBOMe. 
(a) 25C-ortho

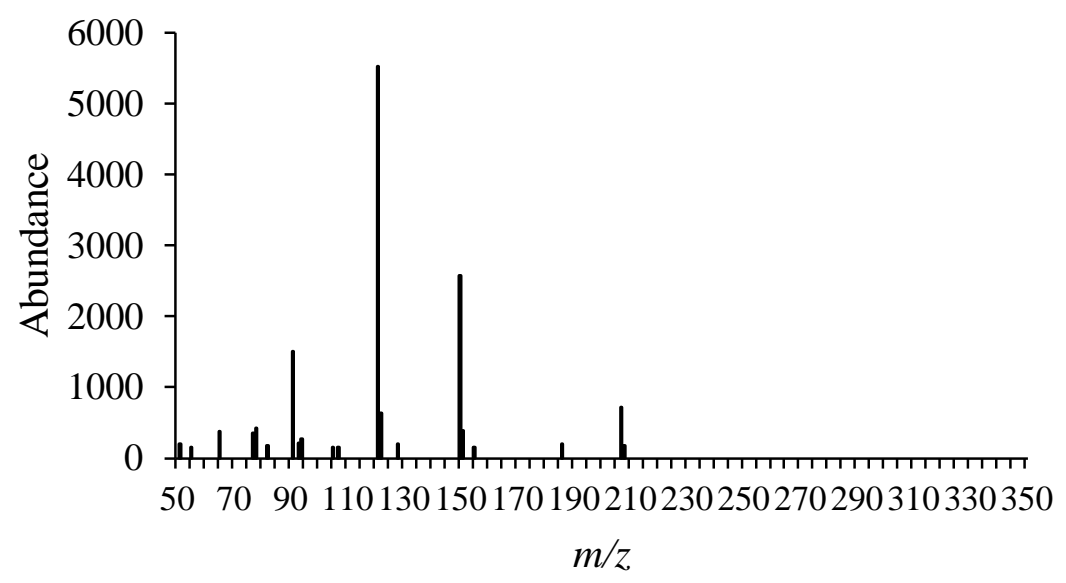

(b) $25 C$-meta

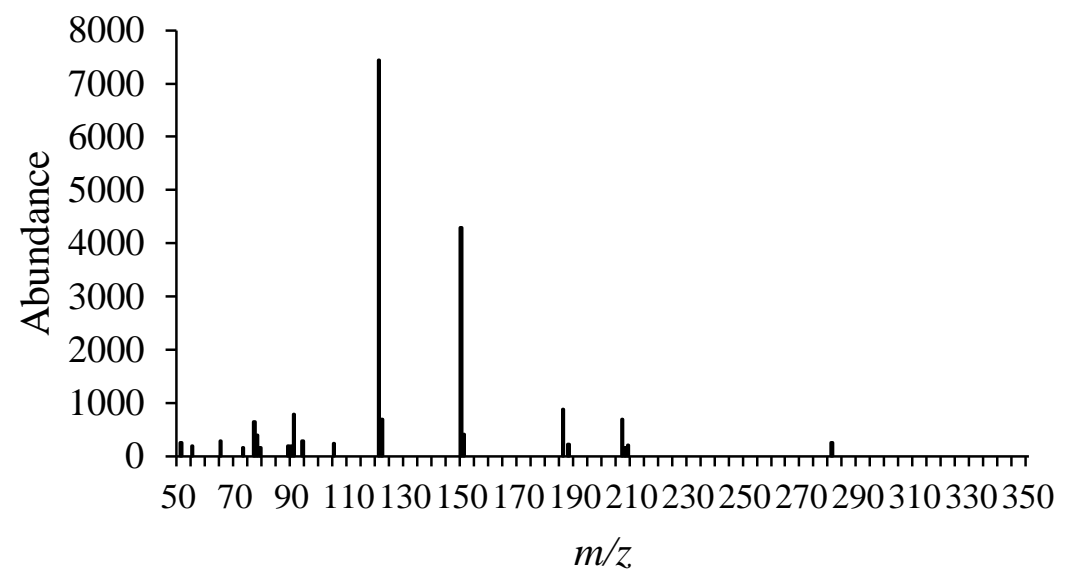

(c) 25C-para

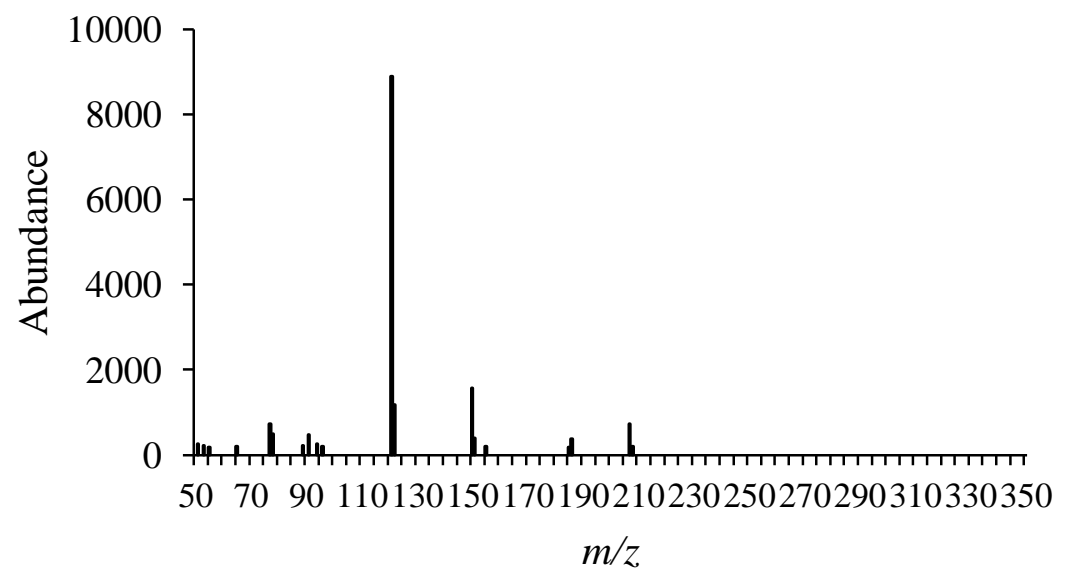

Figure 3.18. 12.5 ppm 25C-NBOMe Agilent fast GC-MS spectra corresponding to $25 C$-ortho (a), 25C-meta (b), and 25C-para (c). 
Figure 3.19 shows the $125 \mathrm{ppm}$ results for the three 25I-NBOMe isomers analyzed with the Agilent instrument under traditional GC-MS conditions. The base peak is $m / z 121$ and there is a possible $\mathrm{M}+2$ peak of the molecular ion at $m / z$ 429. The spectra are truncated from $m / z 50$ to $\mathrm{m} / \mathrm{z} 350$ to eliminate the majority of the contamination peaks. Once again, the relative abundance of the $\mathrm{m} / z, 150$ and $\mathrm{m} / \mathrm{z}, 91$ peaks are of particular importance for the differentiation of the 25I-NBOMe positional isomers. 
(a) 25I-ortho

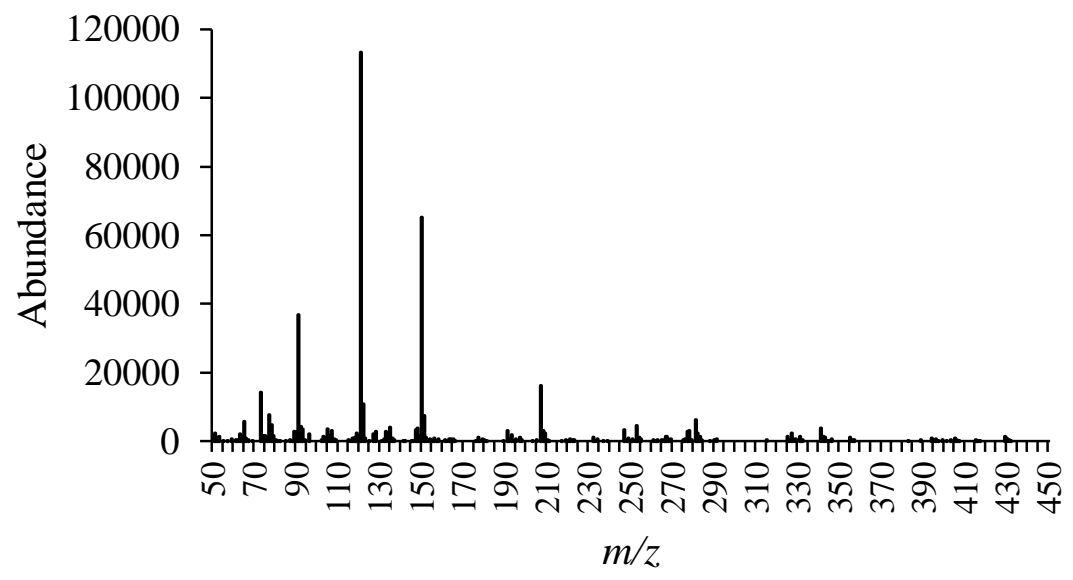

(b) 25I-meta

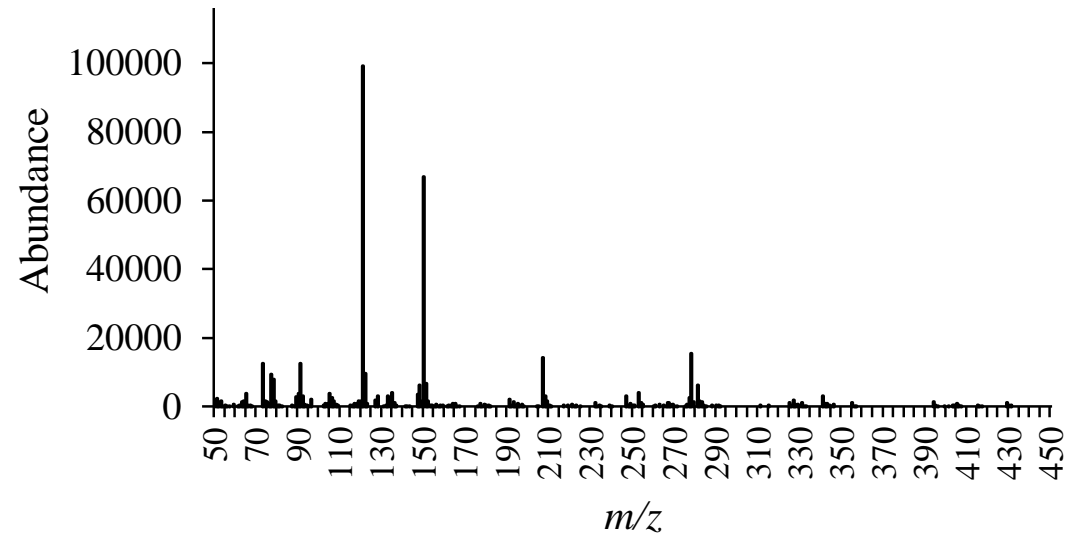

(c) 25I-para

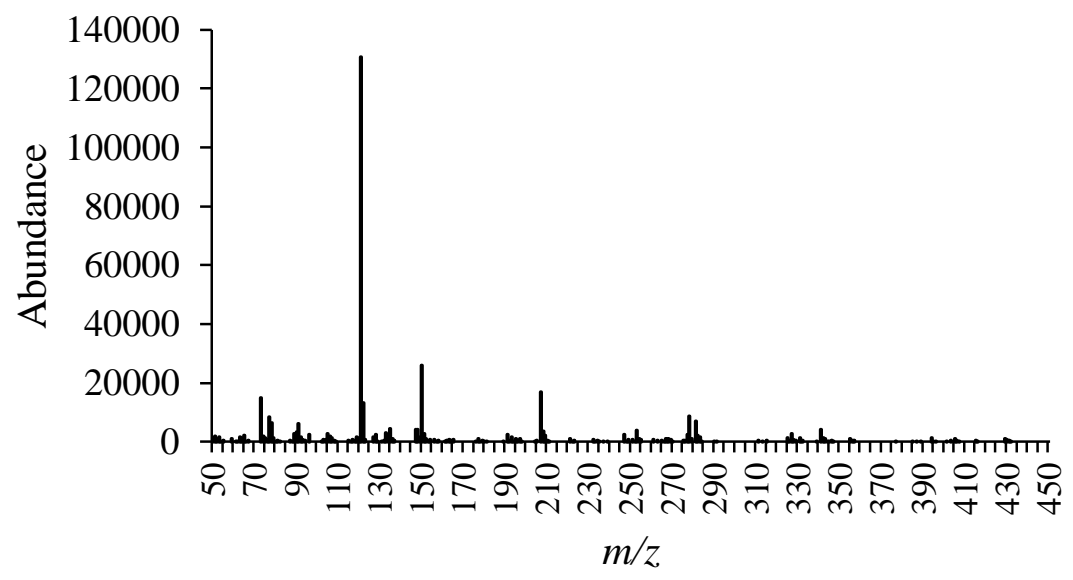

Figure 3.19. 125 ppm 25I-NBOMe Agilent traditional GC-MS spectra corresponding to 25Iortho (a), 25I-meta (b), and 25I-para (c). 
Figure 3.20 shows the 1,250 ppm results for the three 25C-NBOMe isomers analyzed with the PerkinElmer instrument with the use of traditional GC-MS. The base peak is $m / z, 121$ and no molecular ion peak is present in the spectra. The ions at $m / z 150$ and 91 are the second and third most abundant ions in the spectra and the relative abundance is important in the differentiation of the positional isomer of 25C-NBOMe. 
(a) 25C-ortho

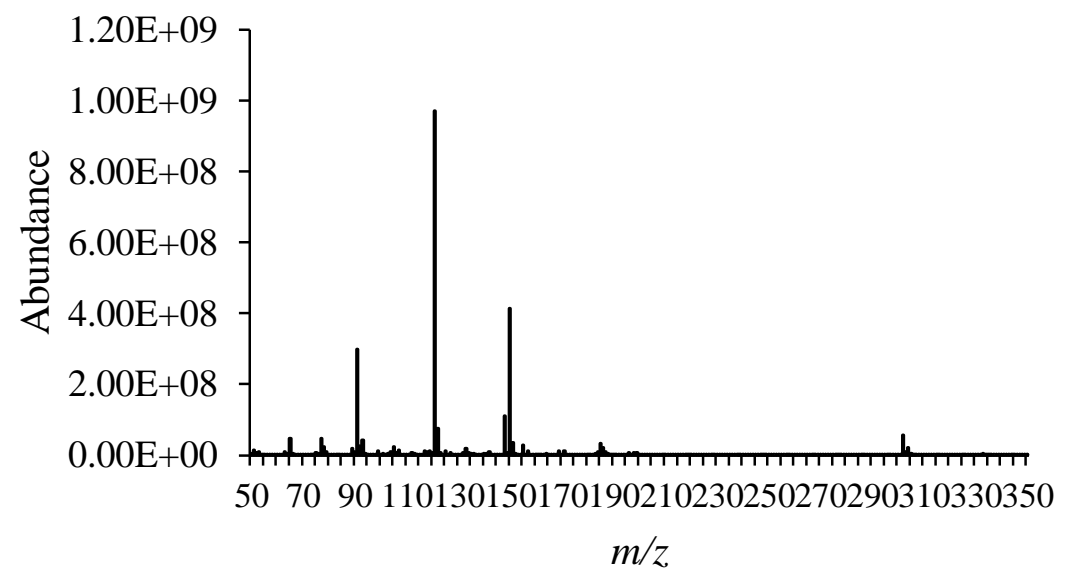

(b) 25C-meta

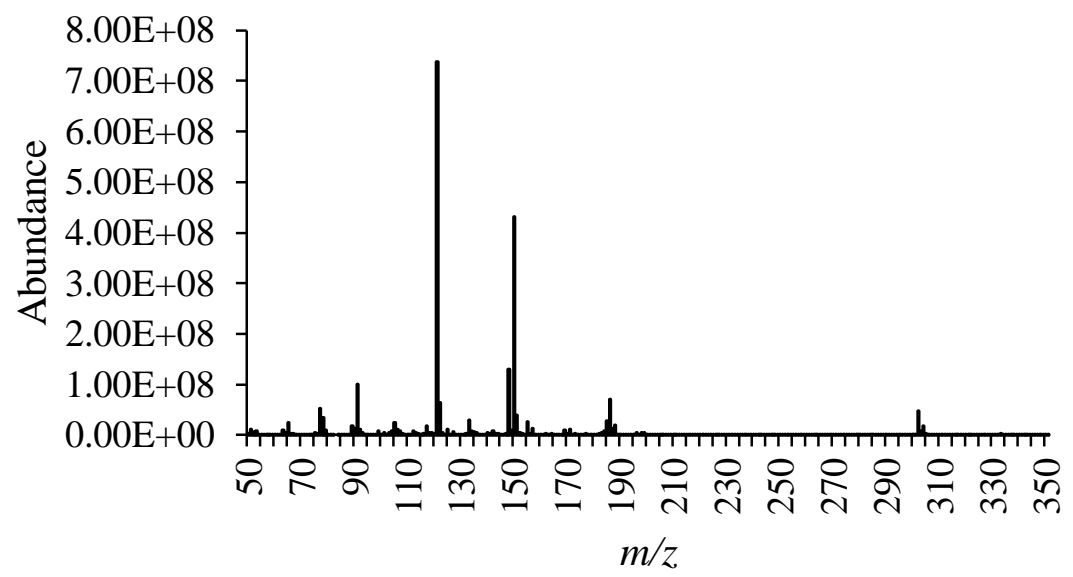

(c) 25C-para

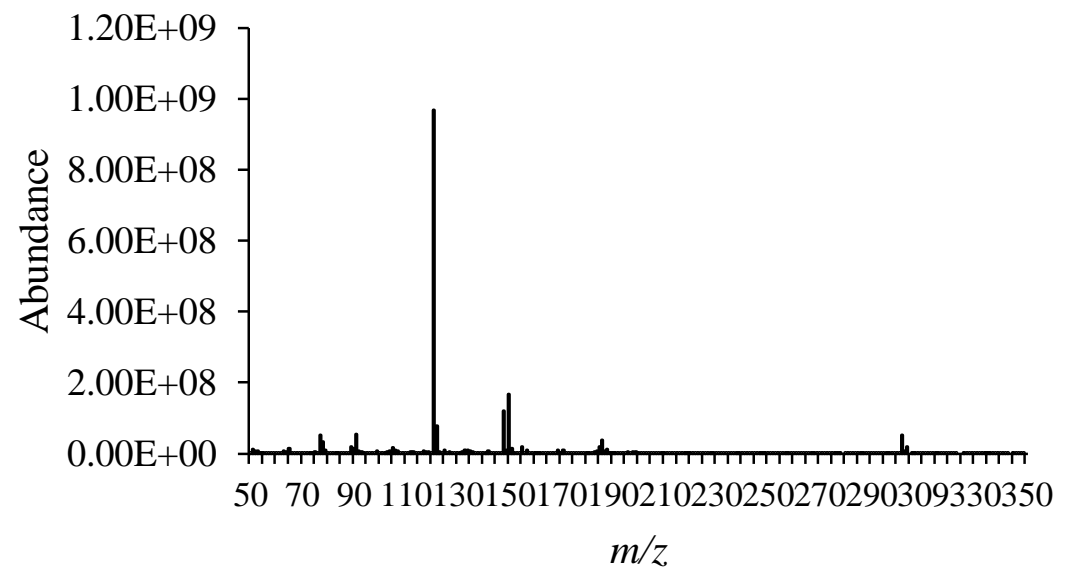

Figure 3.20. 1,250 ppm 25C-NBOMe PerkinElmer traditional GC-MS spectra corresponding to 25C-ortho (a), 25C-meta (b), and 25C-para (c). 
There are several trends of note in the example spectra. First, none of the 25C-NBOMe isomers produced a molecular ion peak, whereas all of the 25I-NBOMe isomers produced a possible M+2 peak at $m / z 429$. These findings were consistent with other works, such as Zuba and Sekula where it was reported that there was a lack of molecular ion peaks seen in GC-MS spectra of NBOMes, and Casale and Hays where it was reported that the molecular ions were observed in relative abundance ranging from $0.05-1.0 \%[\underline{2}, \underline{44}]$. The possible $\mathrm{M}+2$ peak was seen in the work by Casale and Hays, but so was the molecular ion peak at $m / z 427$ [2].

Another trend is that the fragment at $m / z, 91$ was always most abundant for the ortho isomer and least abundant for the para isomer of both 25C-NBOMe and 25I-NBOMe. This phenomenon has been previously described for NBOMe isomers by Casale and Hays and for the synthetic cannabinoid JWH 250 by Harris et al. [45]. Additionally, it was observed that not only were there significant differences in the relative abundance of $\mathrm{m} / \mathrm{z} 150$ and $\mathrm{m} / \mathrm{z} 91$, but that unique ions are present in the spectra that could be used to indicate the presence of 25C-NBOMe vs 25I-NBOMe. The most abundant examples are $\mathrm{m} / \mathrm{z}$ 185/186 in the 25C-NBOMe spectra and $m / z 277 / 278$ in the 25I-NBOMe spectra.

\subsection{MS SPSS Interpretation}

SPSS software was used to evaluate the significance of different factors on the withinfactor to between-factor variance of the ion abundances relative to the $\mathrm{m} / \mathrm{z} 121$ base peak. The factors assessed were the positional isomer, concentration, day of analysis, week of analysis, instrument, the tune profile, and the speed of GC. Once again, the assumptions made by this technique are that there is homogeneity of variance, the populations are normally distributed, and each value is sampled independently. 
Figure 3.21 is an example of a Q-Q plot used to verify the normality of the relative ion abundances. This particular Q-Q plot is from the traditional GC-MS methodology performed on the Agilent instrument and for the $\mathrm{m} / \mathrm{z} 91$ ion of $25 \mathrm{C}$-NBOMe ortho isomer across all three concentrations. The data aligns quite well with the expected normal value, indicating the normality of the data is met.

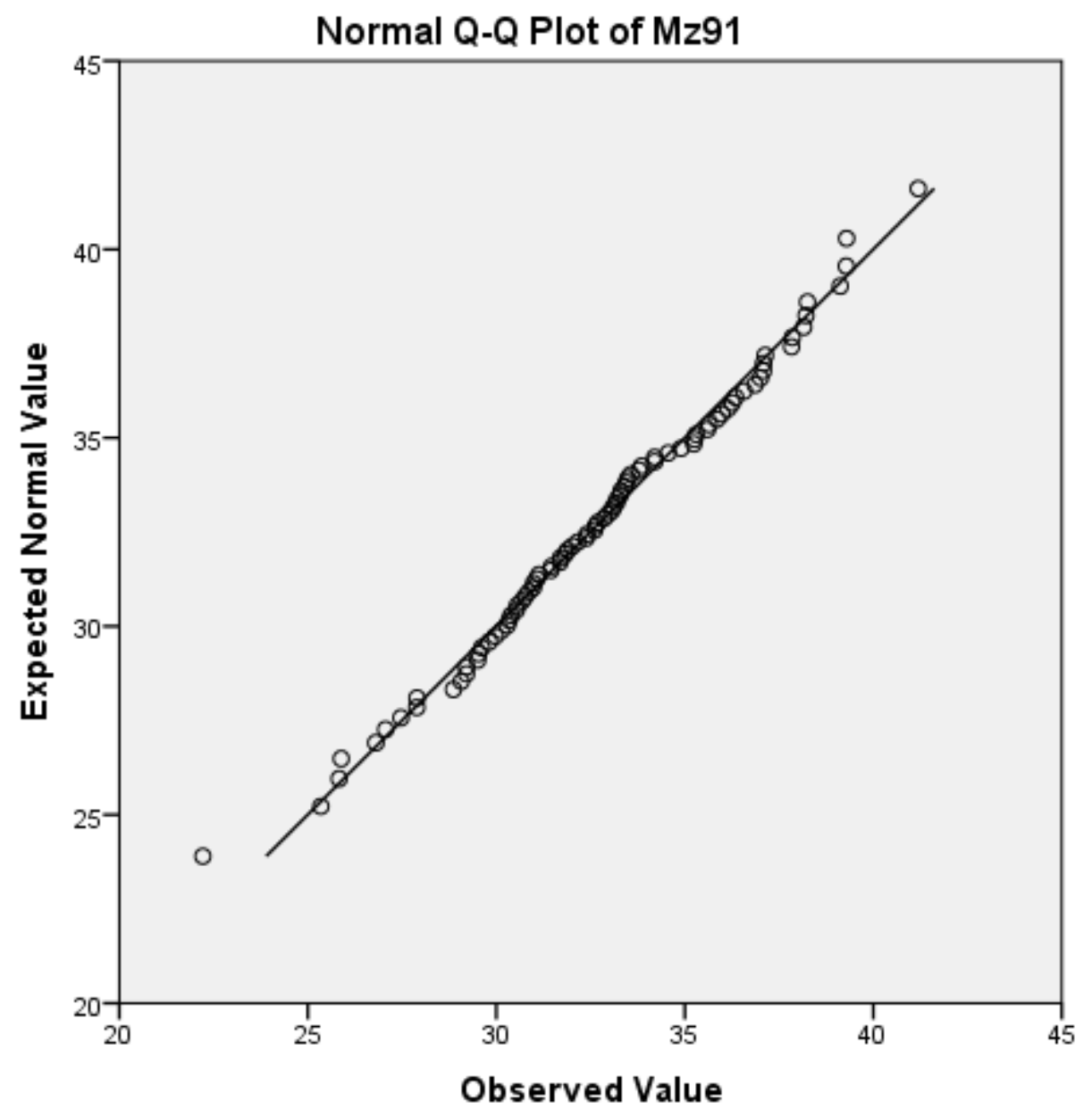

Figure 3.21. $Q-Q$ plot of 12.5 ppm 25C-NBOMe ortho isomer demonstrating normality. 
The homogeneity of variances was also analyzed through the SPSS software and it was determined that not all of the ion abundance data produced homogeneous variance results. However, ANOVA is considered a robust technique and is able to tolerate some of these non-homogeneities, particularly because the sample sizes were approximately equal for comparisons between ions.

The observed variances in the relative ion abundances were analyzed to determine which sources contributed most significantly to the variance. The results for the ANOVA analysis of the relative ion abundances are shown in Table 3.7. The results are provided as p-values. Rows highlighted in gray indicate ions where significant differences are observed.

Table 3.7. ANOVA results ( $p$ values) for the inter-factor variance and intra-factor variance considering four different fixed factors. 15 different fragment ion abundances (normalized to $\mathrm{m} / \mathrm{z}$ 121) were used as the variables.

\begin{tabular}{|l|l|l|l|l|}
\hline $\begin{array}{c}\text { Ions } \\
(\mathrm{m} / \boldsymbol{z})\end{array}$ & Speed of GC & Instrument & Week & Day \\
\hline 65 & $<1.0 \times 10^{-5}$ & $<1.0 \times 10^{-5}$ & $8.9 \times 10^{-2}$ & $5.7 \times 10^{-1}$ \\
\hline 77 & $3.4 \times 10^{-5}$ & $1.3 \times 10^{-4}$ & $7.3 \times 10^{-2}$ & $4.2 \times 10^{-1}$ \\
\hline 78 & $3.4 \times 10^{-2}$ & $1.1 \times 10^{-1}$ & $2.3 \times 10^{-1}$ & $4.6 \times 10^{-1}$ \\
\hline 91 & $3.1 \times 10^{-4}$ & $2.8 \times 10^{-4}$ & $2.9 \times 10^{-1}$ & $4.8 \times 10^{-1}$ \\
\hline 105 & $3.0 \times 10^{-5}$ & $7.3 \times 10^{-5}$ & $1.9 \times 10^{-1}$ & $3.8 \times 10^{-1}$ \\
\hline 122 & $3.5 \times 10^{-1}$ & $1.6 \times 10^{-1}$ & $7.8 \times 10^{-1}$ & $5.8 \times 10^{-1}$ \\
\hline 148 & $3.4 \times 10^{-1}$ & $1.5 \times 10^{-1}$ & $6.4 \times 10^{-1}$ & $5.4 \times 10^{-1}$ \\
\hline 150 & $3.3 \times 10^{-1}$ & $2.6 \times 10^{-1}$ & $5.4 \times 10^{-1}$ & $8.9 \times 10^{-1}$ \\
\hline 151 & $7.3 \times 10^{-1}$ & $9.3 \times 10^{-1}$ & $7.6 \times 10^{-1}$ & $6.4 \times 10^{-1}$ \\
\hline 155 & $3.9 \times 10^{-1}$ & $3.5 \times 10^{-1}$ & $1.9 \times 10^{-1}$ & $6.3 \times 10^{-1}$ \\
\hline 185 & $6.7 \times 10^{-1}$ & $6.0 \times 10^{-1}$ & $5.6 \times 10^{-1}$ & $5.5 \times 10^{-1}$ \\
\hline 186 & $1.4 \times 10^{-1}$ & $2.0 \times 10^{-1}$ & $2.2 \times 10^{-1}$ & $7.6 \times 10^{-1}$ \\
\hline 247 & $2.8 \times 10^{-5}$ & $<1.0 \times 10^{-5}$ & $1.5 \times 10^{-1}$ & $8.6 \times 10^{-1}$ \\
\hline 277 & $1.9 \times 10^{-2}$ & $<1.0 \times 10^{-5}$ & $8.8 \times 10^{-1}$ & $8.8 \times 10^{-1}$ \\
\hline 278 & $<1.0 \times 10^{-5}$ & $<1.0 \times 10^{-5}$ & $1.2 \times 10^{-1}$ & $9.7 \times 10^{-1}$ \\
\hline
\end{tabular}


The ANOVA results indicate that the day of analysis does not have an effect on the relative ion abundances in the mass spectra because none of the p-value results were less than the 0.05 alpha value (for $95 \% \mathrm{CI}$ ). Likewise, the relative ion abundances are not statistically different based on the week of analysis. However, both the speed of GC and instrument factors do show significant differences at the $95 \%$ confidence level between at least six of the ions indicated by the p-values lower than the 0.05 alpha value. This is an indication that significant differences exist between the relative ion abundances due to these factors. The speed of GC and instrument factors are directly linked as the column present in the instrument is responsible for both the speed of GC and the instrument parameters. Due to significant differences in relative ion abundances between instruments, the remaining ANOVA analyses were conducted individually for each analysis type. These results are shown in Table 3.8.

Table 3.8. ANOVA results ( $p$ values) for each analysis method.

\begin{tabular}{|c|c|c|c|c|c|c|c|c|c|}
\hline & \multicolumn{3}{|c|}{ Isomer } & \multicolumn{3}{|c|}{ Concentration } & \multicolumn{3}{|c|}{ Tune } \\
\hline $\begin{array}{l}\text { Ion } \\
(\mathrm{m} / \mathrm{z})\end{array}$ & $\begin{array}{l}\text { Agilent } \\
\text { Fast }\end{array}$ & Agilent & $\mathbf{P E}$ & $\begin{array}{c}\text { Agilent } \\
\text { Fast }\end{array}$ & Agilent & PE & $\begin{array}{l}\text { Agilent } \\
\text { Fast }\end{array}$ & Agilent & $\mathbf{P E}$ \\
\hline 65 & $<1 \times 10^{-5}$ & $<1 \times 10^{-5}$ & $<1 \times 10^{-5}$ & $<1 \times 10^{-5}$ & $<1 \times 10^{-5}$ & $4.9 \times 10^{-4}$ & $9.9 \times 10^{-1}$ & $9.9 \times 10^{-1}$ & $7.4 \times 10^{-1}$ \\
\hline 77 & $<1 \times 10^{-5}$ & $<1 \times 10^{-5}$ & $<1 \times 10^{-5}$ & $<1 \times 10^{-5}$ & $<1 \times 10^{-5}$ & $<1 \times 10^{-5}$ & $8.5 \times 10^{-1}$ & $5.4 \times 10^{-1}$ & $<1 \times 10^{-5}$ \\
\hline 78 & $<1 \times 10^{-5}$ & $<1 \times 10^{-5}$ & $<1 \times 10^{-5}$ & $<1 \times 10^{-5}$ & $<1 \times 10^{-5}$ & $4.3 \times 10^{-5}$ & $9.9 \times 10^{-1}$ & $9.4 \times 10^{-1}$ & $<1 \times 10^{-5}$ \\
\hline 91 & $<1 \times 10^{-5}$ & $<1 \times 10^{-5}$ & $<1 \times 10^{-5}$ & $5.1 \times 10^{-2}$ & $5.4 \times 10^{-2}$ & $1.8 \times 10^{-1}$ & $9.9 \times 10^{-1}$ & $9.9 \times 10^{-1}$ & $9.4 \times 10^{-1}$ \\
\hline 105 & $<1 \times 10^{-5}$ & $<1 \times 10^{-5}$ & $<1 \times 10^{-5}$ & $<1 \times 10^{-5}$ & $<1 \times 10^{-5}$ & $3.6 \times 10^{-3}$ & $9.9 \times 10^{-1}$ & $8.8 \times 10^{-1}$ & $8.1 \times 10^{-1}$ \\
\hline 122 & $2.3 \times 10^{-3}$ & $6.8 \times 10^{-5}$ & $2.9 \times 10^{-1}$ & $<1 \times 10^{-5}$ & $4.5 \times 10^{-4}$ & $6.2 \times 10^{-1}$ & $8.7 \times 10^{-1}$ & $6.5 \times 10^{-1}$ & $9.9 \times 10^{-1}$ \\
\hline 148 & $<1 \times 10^{-5}$ & $<1 \times 10^{-5}$ & $<1 \times 10^{-5}$ & $<1 \times 10^{-5}$ & $<1 \times 10^{-5}$ & $<1 \times 10^{-5}$ & $9.0 \times 10^{-1}$ & $9.1 \times 10^{-2}$ & $<1 \times 10^{-5}$ \\
\hline 150 & $<1 \times 10^{-5}$ & $<1 \times 10^{-5}$ & $<1 \times 10^{-5}$ & $9.9 \times 10^{-1}$ & $2.6 \times 10^{-1}$ & $4.0 \times 10^{-1}$ & 1.0 & 1.0 & $9.0 \times 10^{-1}$ \\
\hline 151 & $<1 \times 10^{-5}$ & $<1 \times 10^{-5}$ & $<1 \times 10^{-5}$ & $<1 \times 10^{-5}$ & $<1 \times 10^{-5}$ & $6.8 \times 10^{-4}$ & $9.9 \times 10^{-1}$ & $9.9 \times 10^{-1}$ & $9.9 \times 10^{-1}$ \\
\hline 155 & $<1 \times 10^{-5}$ & $<1 \times 10^{-5}$ & $<1 \times 10^{-5}$ & $<1 \times 10^{-5}$ & $<1 \times 10^{-5}$ & $2.9 \times 10^{-1}$ & $9.9 \times 10^{-1}$ & $9.9 \times 10^{-1}$ & $1.1 \times 10^{-1}$ \\
\hline 185 & $<1 \times 10^{-5}$ & $<1 \times 10^{-5}$ & $<1 \times 10^{-5}$ & $<1 \times 10^{-5}$ & $<1 \times 10^{-5}$ & $5.2 \times 10^{-1}$ & $9.9 \times 10^{-1}$ & $9.9 \times 10^{-1}$ & $9.8 \times 10^{-1}$ \\
\hline 186 & $<1 \times 10^{-5}$ & $<1 \times 10^{-5}$ & $<1 \times 10^{-5}$ & $<1 \times 10^{-5}$ & $<1 \times 10^{-5}$ & $6.1 \times 10^{-1}$ & $9.9 \times 10^{-1}$ & $9.9 \times 10^{-1}$ & $9.9 \times 10^{-1}$ \\
\hline 247 & $<1 \times 10^{-5}$ & $<1 \times 10^{-5}$ & $<1 \times 10^{-5}$ & $<1 \times 10^{-5}$ & $<1 \times 10^{-5}$ & $2.6 \times 10^{-1}$ & $9.8 \times 10^{-1}$ & $9.8 \times 10^{-1}$ & $9.9 \times 10^{-1}$ \\
\hline 277 & $<1 \times 10^{-5}$ & $<1 \times 10^{-5}$ & $<1 \times 10^{-5}$ & $<1 \times 10^{-5}$ & $<1 \times 10^{-5}$ & $1.4 \times 10^{-1}$ & $9.8 \times 10^{-1}$ & $4.0 \times 10^{-1}$ & $9.7 \times 10^{-1}$ \\
\hline 278 & $<1 \times 10^{-5}$ & $<1 \times 10^{-5}$ & $<1 \times 10^{-5}$ & $6.8 \times 10^{-3}$ & $6.6 \times 10^{-3}$ & $4.7 \times 10^{-2}$ & $9.9 \times 10^{-1}$ & $9.8 \times 10^{-1}$ & $9.7 \times 10^{-1}$ \\
\hline
\end{tabular}


For the isomer factor, the results indicate that the relative ion abundances are significantly different at the $95 \%$ confidence level for all ions in all techniques except the $\mathrm{m} / \mathrm{z}$ 122 ion for the PerkinElmer traditional GC-MS technique. This is to say that the fragment ion abundances within an isomer are significantly smaller than the variance between isomers. Likewise, the concentration has a significant (at the $95 \%$ confidence level) effect on the fragment abundances for all fragments except $m / z 91$ and $m / z 150$ for the Agilent fast GC-MS and Agilent traditional GC-MS analyses. However, the PerkinElmer traditional GC-MS analysis only shows significant differences in concentration for ions $m / z 65,77,78,105,148,151$, and 278.

The last factor evaluated for each technique was the effect of the tune profile on the relative ion abundances. The results, as shown in Table 3.8, indicate that none of the ions show a significant difference in relative abundance at the $95 \%$ confidence level for the Agilent fast GCMS and Agilent traditional GC-MS analyses. However, the PerkinElmer traditional GC-MS analysis does show a significant difference for the fragments at $m / z, 77,78$, and 148 .

In all three of these ANOVA analyses, the PerkinElmer traditional GC-MS analysis produced results different from the Agilent fast and Agilent traditional GC-MS analyses. The difference in mass spectra could be due to natural variations in performance by the mass spectrometers for each respective manufacturer or due to factors outside the model studied. However, another explanation is that the PerkinElmer instrument had a difficult time autotuning correctly. The purpose of an autotune is to generate parameters to maximize performance based on the analysis of an internal standard, in this case PFTBA [46]. It was noted that the consistency with which this particular instrument performed an autotune was quite irregular and this fact was significant enough that another group using this instrument generated its own tune profile to maximize performance. Because one of the goals of this project was to assess the effect of tune 
profile on the mass spectral results, the issue was noted, but the autotune approach was still used.

The results indicate that these inconsistencies between instrumental techniques are likely due to the efficiency with which the mass spectrometer tuned on a day-to-day basis.

The eta squared test is used to determine the proportion of the total variance in a dependent variable that is associated with variation in an independent variable. The relative ion abundance results are shown below in Table 3.9.

Table 3.9. Eta squared ion abundance data.

\begin{tabular}{|c|c|c|c|c|}
\hline $\begin{array}{c}\text { Ions } \\
(\boldsymbol{m} / \boldsymbol{z})\end{array}$ & Speed of GC & Instrument & Week & Day \\
\hline 65 & $2.0 \times 10^{-2}$ & $2.2 \times 10^{-2}$ & $9.0 \times 10^{-3}$ & $9.1 \times 10^{-3}$ \\
\hline 77 & $1.1 \times 10^{-2}$ & $1.2 \times 10^{-2}$ & $8.5 \times 10^{-3}$ & $9.5 \times 10^{-3}$ \\
\hline 78 & $3.0 \times 10^{-3}$ & $3.0 \times 10^{-3}$ & $6.3 \times 10^{-3}$ & $9.3 \times 10^{-3}$ \\
\hline 91 & $8.6 \times 10^{-3}$ & $1.1 \times 10^{-2}$ & $5.6 \times 10^{-3}$ & $9.1 \times 10^{-3}$ \\
\hline 105 & $1.3 \times 10^{-2}$ & $1.4 \times 10^{-2}$ & $7.6 \times 10^{-3}$ & $1.1 \times 10^{-2}$ \\
\hline 122 & $5.8 \times 10^{-4}$ & $2.4 \times 10^{-3}$ & $2.6 \times 10^{-3}$ & $8.1 \times 10^{-3}$ \\
\hline 148 & $7.0 \times 10^{-4}$ & $2.8 \times 10^{-3}$ & $3.9 \times 10^{-3}$ & $1.0 \times 10^{-2}$ \\
\hline 150 & $6.2 \times 10^{-4}$ & $1.8 \times 10^{-3}$ & $3.9 \times 10^{-3}$ & $5.2 \times 10^{-3}$ \\
\hline 151 & $8.6 \times 10^{-5}$ & $1.0 \times 10^{-4}$ & $2.9 \times 10^{-3}$ & $8.0 \times 10^{-3}$ \\
\hline 155 & $7.3 \times 10^{-4}$ & $2.0 \times 10^{-3}$ & $9.8 \times 10^{-3}$ & $1.1 \times 10^{-2}$ \\
\hline 185 & $2.0 \times 10^{-4}$ & $1.1 \times 10^{-3}$ & $6.3 \times 10^{-3}$ & $1.4 \times 10^{-2}$ \\
\hline 186 & $2.2 \times 10^{-3}$ & $3.2 \times 10^{-3}$ & $9.4 \times 10^{-3}$ & $1.0 \times 10^{-2}$ \\
\hline 247 & $2.1 \times 10^{-2}$ & $1.0 \times 10^{-2}$ & $1.3 \times 10^{-2}$ & $1.0 \times 10^{-2}$ \\
\hline 277 & $6.8 \times 10^{-3}$ & $4.8 \times 10^{-2}$ & $3.8 \times 10^{-3}$ & $1.0 \times 10^{-2}$ \\
\hline 278 & $4.1 \times 10^{-2}$ & $1.1 \times 10^{-2}$ & $1.3 \times 10^{-2}$ & $6.7 \times 10^{-3}$ \\
\hline
\end{tabular}

The results in Table 3.9 indicate that the day of analysis contributes only minimally to the variation in the relative ion abundances as the values range from $0.5-1.4 \%$. Likewise, the week of analysis did not contribute significantly to the variation in the relative ion abundances as these values range between $0.3-1.3 \%$. This same conclusion can be reached for both the speed of GC and instrument factors as the range of values was $0.1-4.1 \%$ and $0.1-4.8 \%$, respectively.

The significant differences in the relative ion abundance ANOVA results for the instrument and speed of GC indicated that the eta squared test should be performed individually 
for each technique. The results for these tests are shown in Table 3.10. Rows highlighted in gray indicate significant contributions to the total variance were present.

Table 3.10. Eta squared ion relative abundance data for each analysis method.

\begin{tabular}{|c|c|c|c|c|c|c|c|c|c|}
\hline & \multicolumn{3}{|c|}{ Isomer } & \multicolumn{3}{|c|}{ Concentration } & \multicolumn{3}{|c|}{ Tune } \\
\hline Ions & $\begin{array}{c}\text { Agilent } \\
\text { Fast }\end{array}$ & Agilent & PE & $\begin{array}{l}\text { Agilent } \\
\text { Fast }\end{array}$ & Agilent & PE & $\begin{array}{c}\text { Agilent } \\
\text { Fast }\end{array}$ & Agilent & PE \\
\hline 65 & $6.8 \times 10^{-1}$ & $7.1 \times 10^{-1}$ & $7.5 \times 10^{-1}$ & $3.9 \times 10^{-1}$ & $1.1 \times 10^{-1}$ & $3.1 \times 10^{-2}$ & $8.6 \times 10^{-3}$ & $7.8 \times 10^{-3}$ & $2.0 \times 10^{-2}$ \\
\hline 77 & $1.9 \times 10^{-1}$ & $1.7 \times 10^{-1}$ & $2.0 \times 10^{-1}$ & $3.5 \times 10^{-1}$ & $4.3 \times 10^{-1}$ & $6.5 \times 10^{-2}$ & $1.7 \times 10^{-2}$ & $2.5 \times 10^{-2}$ & $1.3 \times 10^{-1}$ \\
\hline 78 & $4.4 \times 10^{-1}$ & $2.4 \times 10^{-1}$ & $3.7 \times 10^{-1}$ & $3.1 \times 10^{-1}$ & $4.6 \times 10^{-1}$ & $1.1 \times 10^{-2}$ & $9.0 \times 10^{-3}$ & $1.4 \times 10^{-2}$ & $1.1 \times 10^{-1}$ \\
\hline 91 & $9.7 \times 10^{-1}$ & $9.4 \times 10^{-1}$ & $8.9 \times 10^{-1}$ & $1.2 \times 10^{-2}$ & $1.1 \times 10^{-2}$ & $7.2 \times 10^{-3}$ & $2.0 \times 10^{-3}$ & $2.1 \times 10^{-3}$ & $1.3 \times 10^{-2}$ \\
\hline 105 & $3.0 \times 10^{-1}$ & $4.2 \times 10^{-1}$ & $5.8 \times 10^{-1}$ & $4.3 \times 10^{-1}$ & $3.0 \times 10^{-1}$ & $2.3 \times 10^{-2}$ & $1.3 \times 10^{-2}$ & $1.8 \times 10^{-2}$ & $1.8 \times 10^{-2}$ \\
\hline 122 & $3.5 \times 10^{-2}$ & $5.1 \times 10^{-2}$ & $1.3 \times 10^{-2}$ & $1.1 \times 10^{-1}$ & $3.0 \times 10^{-2}$ & $2.0 \times 10^{-3}$ & $1.6 \times 10^{-2}$ & $2.2 \times 10^{-2}$ & $4.2 \times 10^{-3}$ \\
\hline 148 & $2.7 \times 10^{-1}$ & $1.3 \times 10^{-1}$ & $1.1 \times 10^{-1}$ & $3.6 \times 10^{-1}$ & $3.2 \times 10^{-1}$ & $3.6 \times 10^{-1}$ & $8.9 \times 10^{-3}$ & $4.4 \times 10^{-2}$ & $1.8 \times 10^{-1}$ \\
\hline 150 & $9.7 \times 10^{-1}$ & $9.6 \times 10^{-1}$ & $8.8 \times 10^{-1}$ & $3.5 \times 10^{-5}$ & $5.0 \times 10^{-3}$ & $3.8 \times 10^{-3}$ & $7.6 \times 10^{-3}$ & $6.8 \times 10^{-3}$ & $1.5 \times 10^{-2}$ \\
\hline 151 & $8.5 \times 10^{-1}$ & $8.1 \times 10^{-1}$ & $6.7 \times 10^{-1}$ & $1.0 \times 10^{-1}$ & $8.8 \times 10^{-2}$ & $3.0 \times 10^{-2}$ & $4.8 \times 10^{-3}$ & $6.5 \times 10^{-3}$ & $7.5 \times 10^{-3}$ \\
\hline 155 & $8.7 \times 10^{-1}$ & $8.4 \times 10^{-1}$ & $2.5 \times 10^{-1}$ & $2.0 \times 10^{-1}$ & $1.8 \times 10^{-1}$ & $5.6 \times 10^{-3}$ & $2.0 \times 10^{-2}$ & $7.9 \times 10^{-3}$ & $4.4 \times 10^{-2}$ \\
\hline 185 & $4.9 \times 10^{-1}$ & $9.1 \times 10^{-1}$ & $7.7 \times 10^{-1}$ & $2.7 \times 10^{-1}$ & $1.9 \times 10^{-1}$ & $3.2 \times 10^{-3}$ & $1.0 \times 10^{-2}$ & $7.5 \times 10^{-3}$ & $1.2 \times 10^{-2}$ \\
\hline 186 & $9.5 \times 10^{-1}$ & $9.6 \times 10^{-1}$ & $8.7 \times 10^{-1}$ & $1.1 \times 10^{-1}$ & $1.2 \times 10^{-1}$ & $2.5 \times 10^{-3}$ & $6.9 \times 10^{-3}$ & $6.8 \times 10^{-3}$ & $6.7 \times 10^{-3}$ \\
\hline 247 & $5.3 \times 10^{-1}$ & $9.0 \times 10^{-1}$ & $7.7 \times 10^{-1}$ & $3.1 \times 10^{-1}$ & $1.4 \times 10^{-1}$ & $6.8 \times 10^{-3}$ & $3.0 \times 10^{-2}$ & $2.0 \times 10^{-2}$ & $1.1 \times 10^{-2}$ \\
\hline 277 & $3.7 \times 10^{-1}$ & $8.7 \times 10^{-1}$ & $6.1 \times 10^{-1}$ & $2.4 \times 10^{-1}$ & $1.1 \times 10^{-1}$ & $1.0 \times 10^{-2}$ & $3.1 \times 10^{-2}$ & $6.3 \times 10^{-2}$ & $1.4 \times 10^{-2}$ \\
\hline 278 & $9.1 \times 10^{-1}$ & $9.1 \times 10^{-1}$ & $7.9 \times 10^{-1}$ & $4.1 \times 10^{-2}$ & $5.7 \times 10^{-2}$ & $1.7 \times 10^{-2}$ & $8.5 \times 10^{-3}$ & $2.2 \times 10^{-2}$ & $1.4 \times 10^{-2}$ \\
\hline
\end{tabular}

The results for the isomer factor analysis indicate that the isomer factor contributes significantly to the variation in the relative ion abundances. The Agilent fast GC-MS range is 3.5-97\%, the Agilent traditional GC-MS range is 5.1-96\%, and the PerkinElmer traditional GCMS range is $1.3-88 \%$. The large amount of variance explained in the relative ion abund ance due to variation in the isomers indicates that the impact of the isomer on the relative ion abundances is significant. For example, the rows highlighted in gray in Table 3.10 correspond with the four largest amounts of variance explained in the relative ion abundance due to variation in the isomer. Each of these percentages ranged from $79-97 \%$ based on the technique used for analysis.

The concentration also plays a moderately significant role for several of these techniques as the range of variation in the relative ion abundance explained by variation in the concentration for the Agilent fast GC-MS analysis is $1.2-43 \%$, the Agilent traditional GC-MS range is 0.5$46 \%$, and the PerkinElmer traditional range is $0.2-36 \%$. These results indicate that the isomer 
factor contributed more significantly to the variation in the relative ion abundances than the concentration, but both factors do have an impact.

The eta squared results for the contribution of the tune profile to the variation in the relative ion abundances are of particular significance. The results indicate that the tune profile used for analysis does not contribute significantly to the variation in the relative ion abundances as the range for the Agilent fast GC-MS analysis is $0.1-3.1 \%$, the range for the Agilent traditional GC-MS analysis is $0.2-6.3 \%$, and the range for the PerkinElmer traditional analysis is $0.7-18 \%$. The $18 \%$ variation is observed for $m / z 148$, which may have been naturally enhanced through column or septum bleed over the course of this project.

The reason these results are of particular significance is that the tune profile aspect of the project may hold the most significance towards the application to crime labs. In a conventional crime lab, the analyst performs an autotune on a set schedule according to the lab's standard operating procedure. The question that these results attempt to answer is whether or not the autotune has a significant effect on the analytical results obtained, i.e., would the results be significantly different between two analyses performed on different tune profiles? Based on these results, the answer is no as only a small percentage of the variation in the relative ion abundances is explained by changes in the autotune profiles. 


\subsection{MS Results}

Figure 3.22 shows the PCA plot for the relative ion abundances for all concentrations and a plot of the ion correlation values. The PCA plot demonstrates the natural clustering of the relative ion abundances based on the isomer factor. The eigenvalue for $\mathrm{PC} 1$ is 465.8 and $70.9 \%$ of the variance is captured by this principal component. The eigenvalue for PC 2 is 108.3 and $16.5 \%$ of the variance is captured. With only these two principal components, $87.4 \%$ of the total variance has been captured. The ion correlation plot shows which ions have the strongest correlation with the PCA plot. For example, the $m / z, 150$ correlation value is the largest with respect to function 1 and correlates the strongest with the $25 \mathrm{C}$-ortho and $25 \mathrm{I}$-ortho isomers. Similarly, $m / z 278$ has a large correlation value with respect to function 2 and correlates the strongest with the 25I-NBOMe positional isomers. 

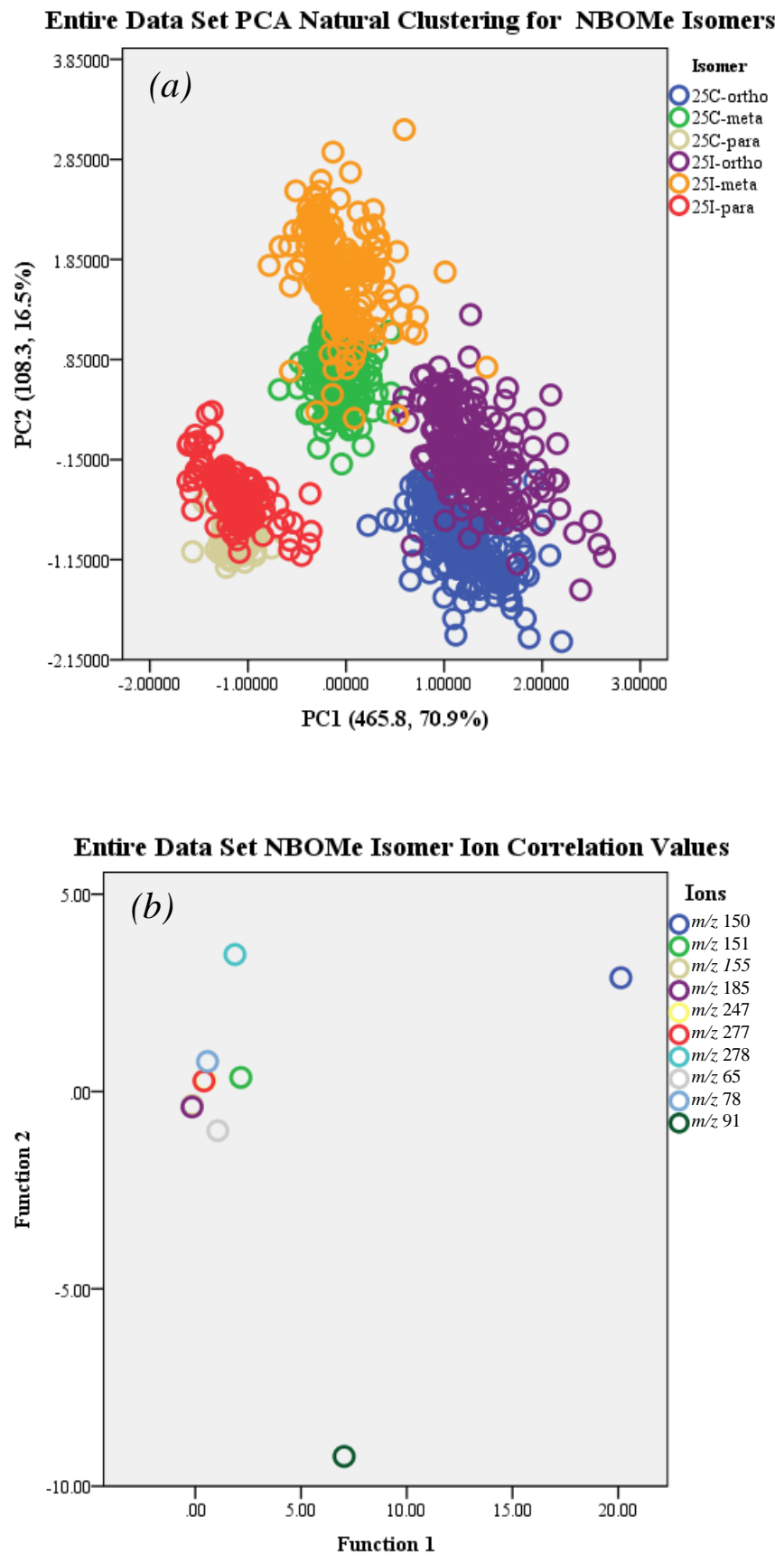

Figure 3.22. Principal Component Analysis (PCA) showing the natural clustering of the NBOMe isomers (a) and the component correlation matrix showing the relationship between each ion and the NBOMe isomer groupings where function 1 and 2 are estimates of the correlations between each of the variables and the estimated components $(b) . N=1515$ for the entire data set. 
Figure 3.23 show the CDA plot for the relative ion abundances for all concentrations and the plot of the ion correlation values. The CDA plot shows the classification of the relative ion abundances into six isomer groupings. The group centroids provide an indication of the variance around the group mean. Function 1 has an eigenvalue of 32.7 and captures $51.8 \%$ of the total variance, while function 2 has an eigenvalue of 17.5 and captures $27.8 \%$ of the variance. In total, $79.6 \%$ of the total variance of the data set was captured with only two discriminant functions. The ion correlation plot shows the correlation between each ion and the six isomer groupings. The ion correlation plot for the CDA results has a better spread of correlation values, which align quite well with the separation of the isomer groupings. For example, $m / z 278$ has the strongest negative function two, which correlates with the 25I-NBOMe positional isomers and $\mathrm{m} / \mathrm{z} 186$ has the strongest positive function two, which correlates with the 25C-NBOMe positional isomers. 
Entire Data Set Inter-Isomer Variability
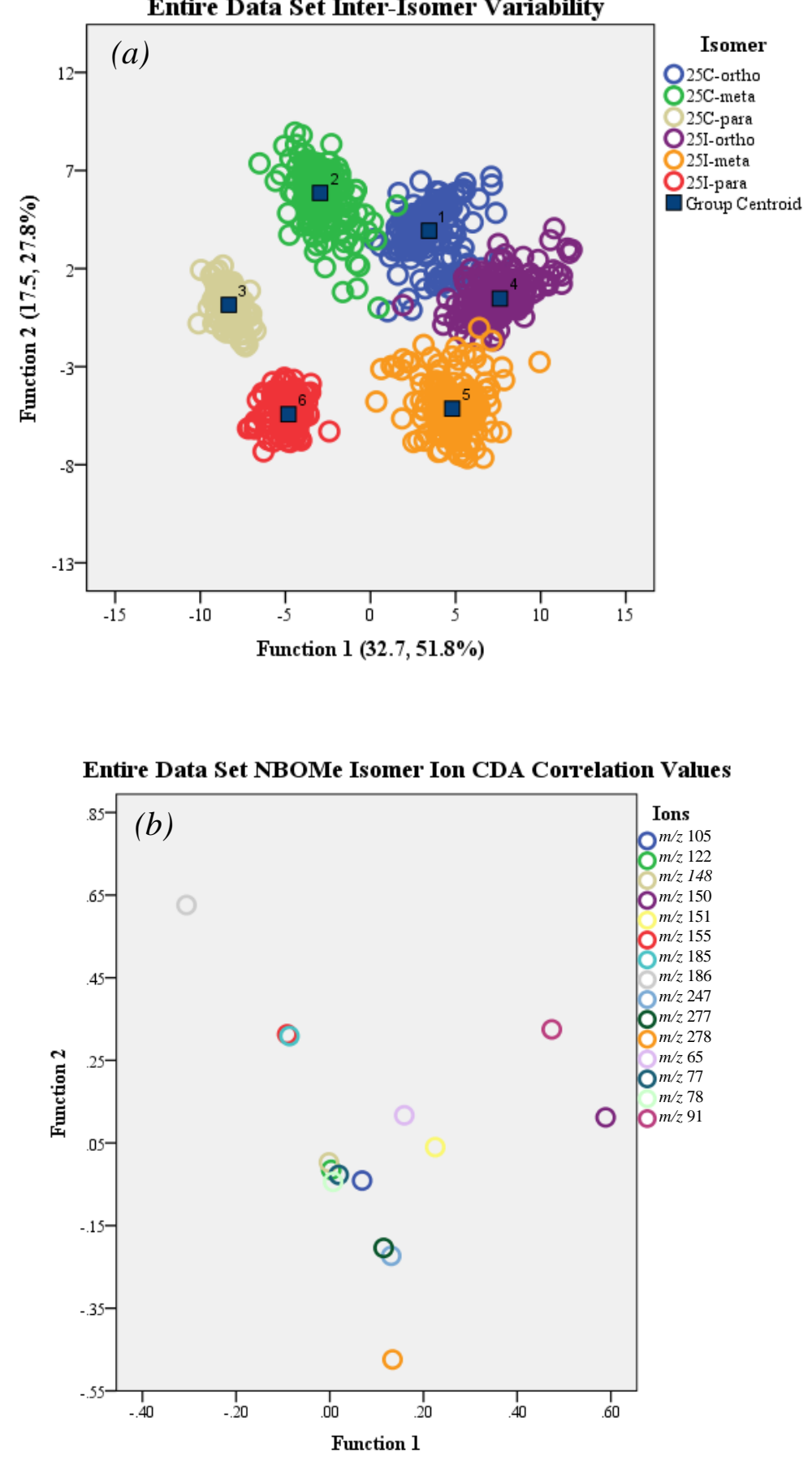

Figure 3.23. Canonical Discriminant Analysis (CDA) showing the classification of the NBOMe isomers based on the relative ion abundances (a) and the structure matrix correlation values where function 1 and 2 are the pooled within group correlations between the discriminant variables and the standardized canonical discriminant functions $(b) . N=1515$ for the entire data set. 
SPSS software was used to generate classification results based on the predicted group membership for both the original and the cross-validated grouped cases. The results, when all three sample concentrations were included, were that $99.5 \%$ of the cases were correctly classified for both the original and cross-validated groupings. However, based on the spread of the natural clustering seen in the PCA plot and the variation observed in the CDA plot, the relative ion abundances corresponding to the lowest concentration samples were removed from the data set and an premium data set consisting of only the $125 \mathrm{ppm}$ and 1,250 ppm samples was created. The reason for the exclusion of the lowest concentration samples was because this is where the most intra-isomer variation was observed.

The PCA plot for the premium data set consisting of the $125 \mathrm{ppm}$ and 1,250 ppm samples is shown in Figure 3.24. This plot shows the natural clustering of the relative ion abundances based on the isomer factor. The eigenvalue for PC1 is 450.8 and $72.1 \%$ of the total variance is captured by this principal component. The eigenvalue for PC2 is 104.7 and $16.8 \%$ of the total variance is captured by this principal component. The combination of these two principal components captures $88.9 \%$ of the total variance in the data set. Again, the ion correlation plot shows the relationship between each ion and the natural clustering observed in the PCA plot. Ion correlations of note are $m / z 91$ and $m / z 150$, which have the strongest function 2 and function 1 correlations, respectively. The $m / z 91$ ion strongly correlates with the 25C-para and 25I-para isomers, while the $\mathrm{m} / \mathrm{z} 150$ ion strongly correlates with $25 \mathrm{C}$-meta and 25I-meta isomers. 

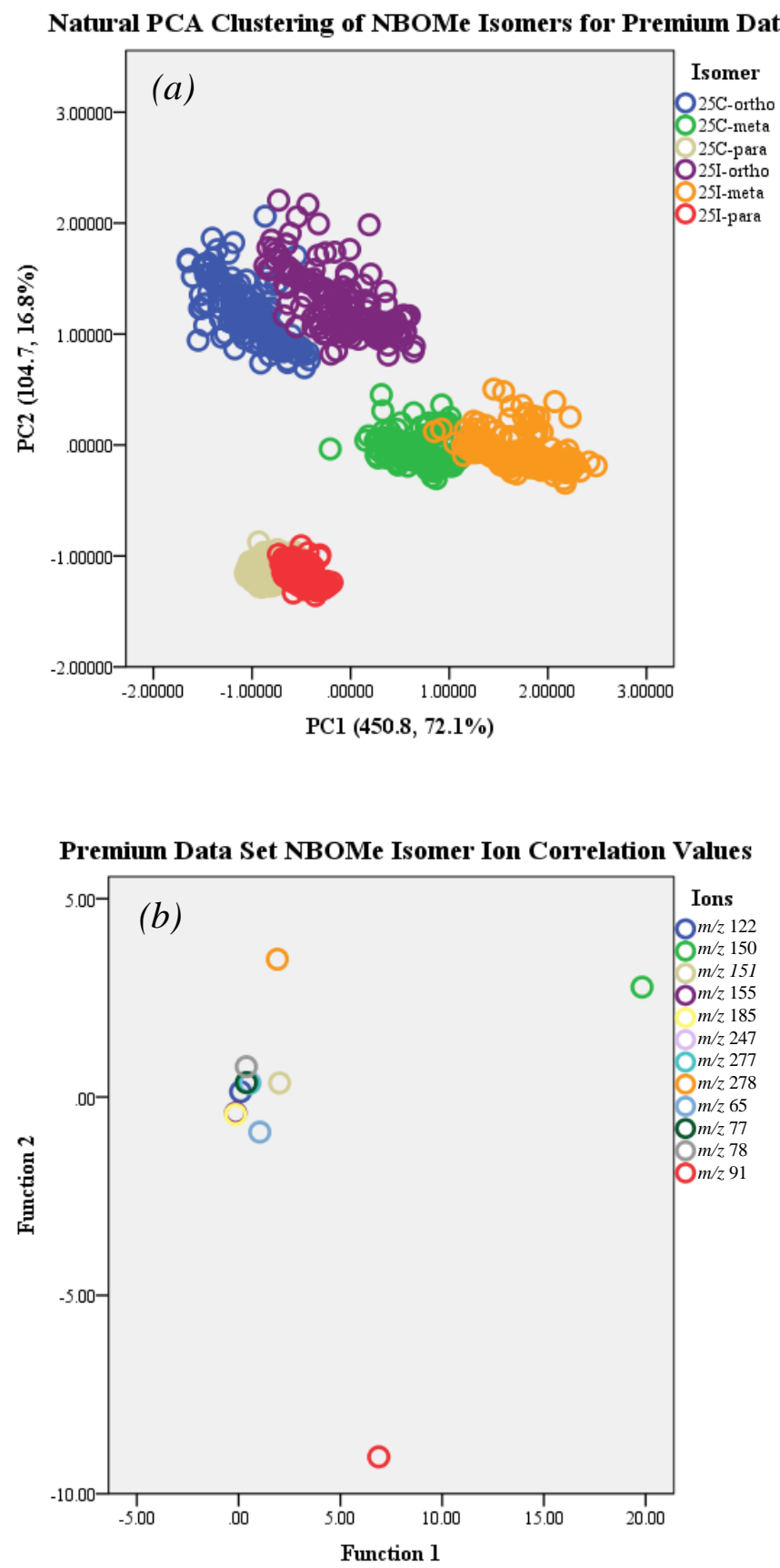

Figure 3.24. Principal Component Analysis (PCA) showing the natural clustering of the NBOMe isomers (a) and the component correlation matrix showing the relationship between each ion and the NBOMe isomer groupings where function 1 and 2 are estimates of the correlations between each of the variables and the estimated components $(b) . N=1029$ for the premium data set which consisted of the $125 \mathrm{ppm}$ and 1,250 ppm samples. 
The CDA plot for the relative ion abundances for the premium data set and the plot of the ion correlation values are shown in Figure 3.25. Once again, the CDA plot shows the classification of the relative ion abundances into six isomer groupings. The eigenvalue for function 1 is 115.6 and $55.4 \%$ of the variance is captured by this discriminant function. The eigenvalue for the second discriminant function is 50.0 and $24.0 \%$ of the variance is captured. A total of $79.4 \%$ of the variance of the data set is captured by these two discriminant functions.

The variance for each isomer grouping relative to the group centroid can be observed and in comparison to the entire data set CDA plot there is more separation between groups. The ion correlation plot for the premium data set CDA results has a wider spread of correlation values as compared to the PCA correlation plot. Again, the relationship between the ion correlations and isomer groupings is apparent as the 25C-NBOMe and 25I-NBOMe positional isomers are separated in relation to the $\mathrm{m} / \mathrm{z} 186$ and $\mathrm{m} / \mathrm{z} 278$ ions and the ortho, meta, and para isomers of both NBOMe compounds are separated in relation to the $m / z 91$ and $m / z 150$. 


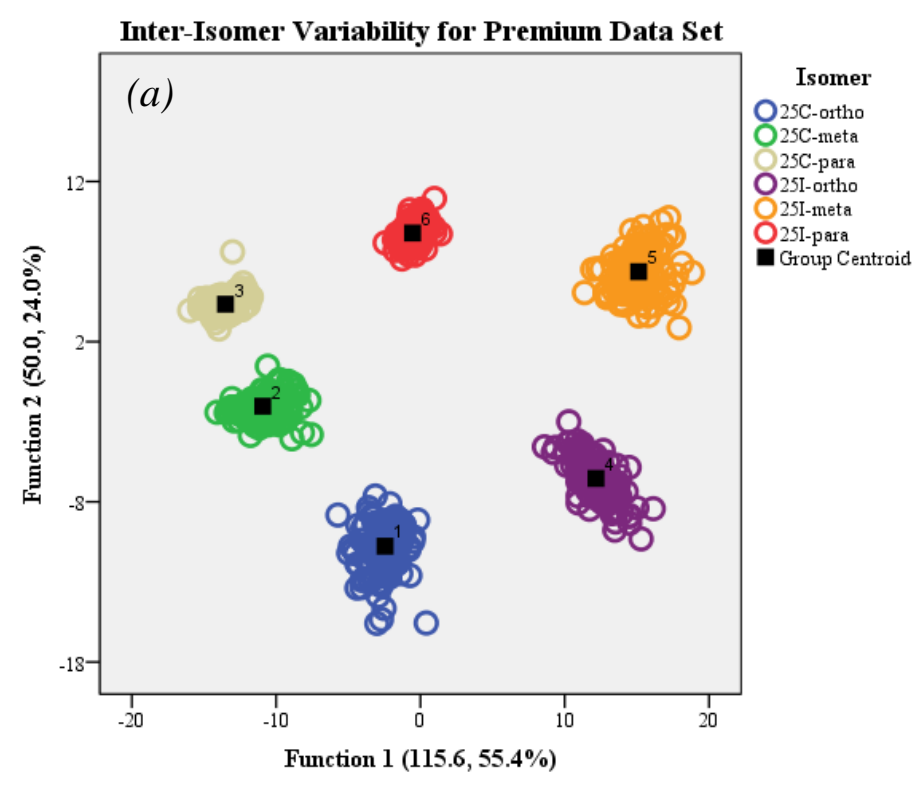

Premium Data Set NBOMe Isomer Ion CDA Correlation Values

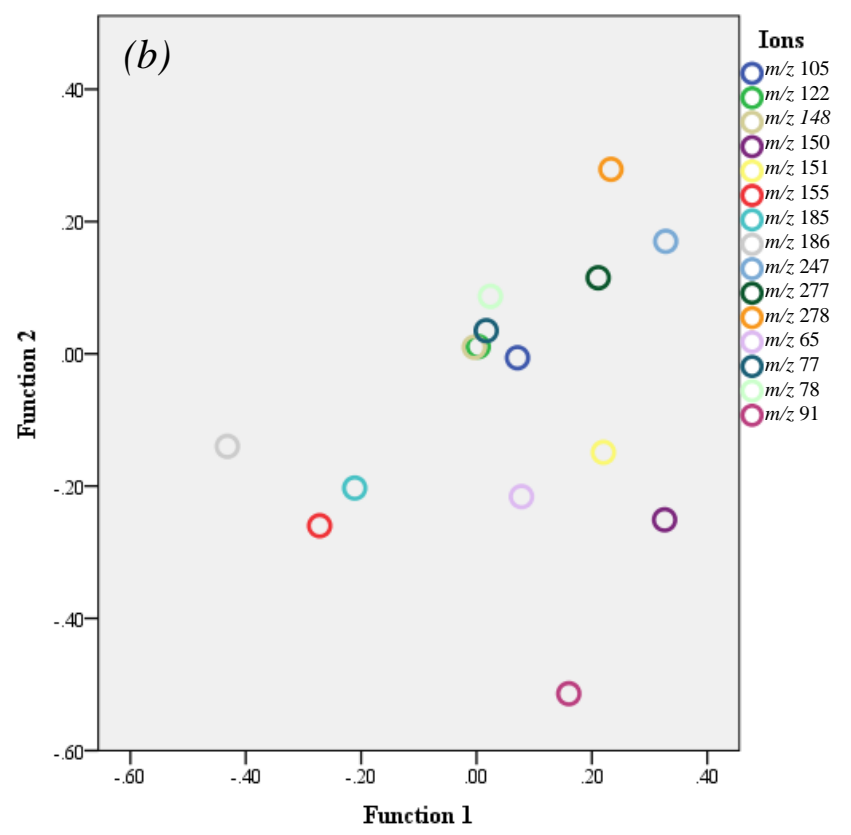

Figure 3.25. Canonical Discriminant Analysis (CDA) showing the classification of the NBOMe isomers based on the relative ion abundances $(a)$ and the structure matrix correlation values where function 1 and 2 are the pooled within group correlations between the discriminant variables and the standardized canonical discriminant functions $(b) . N=1029$ for the premium data set, which contains the 125 ppm and 1,250 ppm samples. 
The classification results based on the predicted group membership for both the original and the cross-validated grouped cases were generated using the SPSS software. The classification results for the premium data set were that $100.0 \%$ of the original grouped cases were classified correctly and $99.9 \%$ of the cross-validated grouped cases were classified correctly. This is an improvement from the original data set classification and indicates that when the higher concentration, or less variable intra-isomer relative ion abundances are used the classification rate increases to a near perfect level.

An additional assessment of the intra-instrument variability of the relative ion abundances for the entire data set with the use of a CDA plot and ion correlation plot is shown in Figure 3.26. The CDA plot contains the relative ion abundance data for only the Agilent fast GCMS methodology. The CDA plot shows the classification of the relative ion abundances into six isomer groupings, where the spread of the data around the group centroids is an indication of the variance for each classification. The spread of the data around the group centroids for the entire data set when all three instrumental methodologies are combined, as seen in Figure 3.23, is greater than when only a single instrumental analysis is analyzed. This reduction in the spread of the data around the group centroid indicates there is less variance in the relative ion abundance data within a single instrumental analysis as compared to when all methodologies are combined.

Function 1 of the CDA plot has an eigenvalue of 51.7 and captures $44.6 \%$ of the variance, while function 2 has an eigenvalue of 35.8 and captures $30.8 \%$ of the variance. In total, $75.4 \%$ of the total variance of the data set was captured with only two discriminant functions. The ion correlation plot shows the correlation between each ion and the six isomer groupings. The ions at $m / z 150, m / z 91, m / z, 278$, and $m / z, 186$ were again identified as the most influential on the classification of NBOMe isomers based on the relative ion abundances. 
The classification results based on the predicted group membership for the original grouped cases and cross-validated grouped cases was $99.6 \%$ and $99.4 \%$, respectively. Once more, the creation of a premium data set that excluded the lowest concentration samples that contained the highest variance was generated in an attempt to increase the classification rate of NBOMe isomers based on relative ion abundances. 

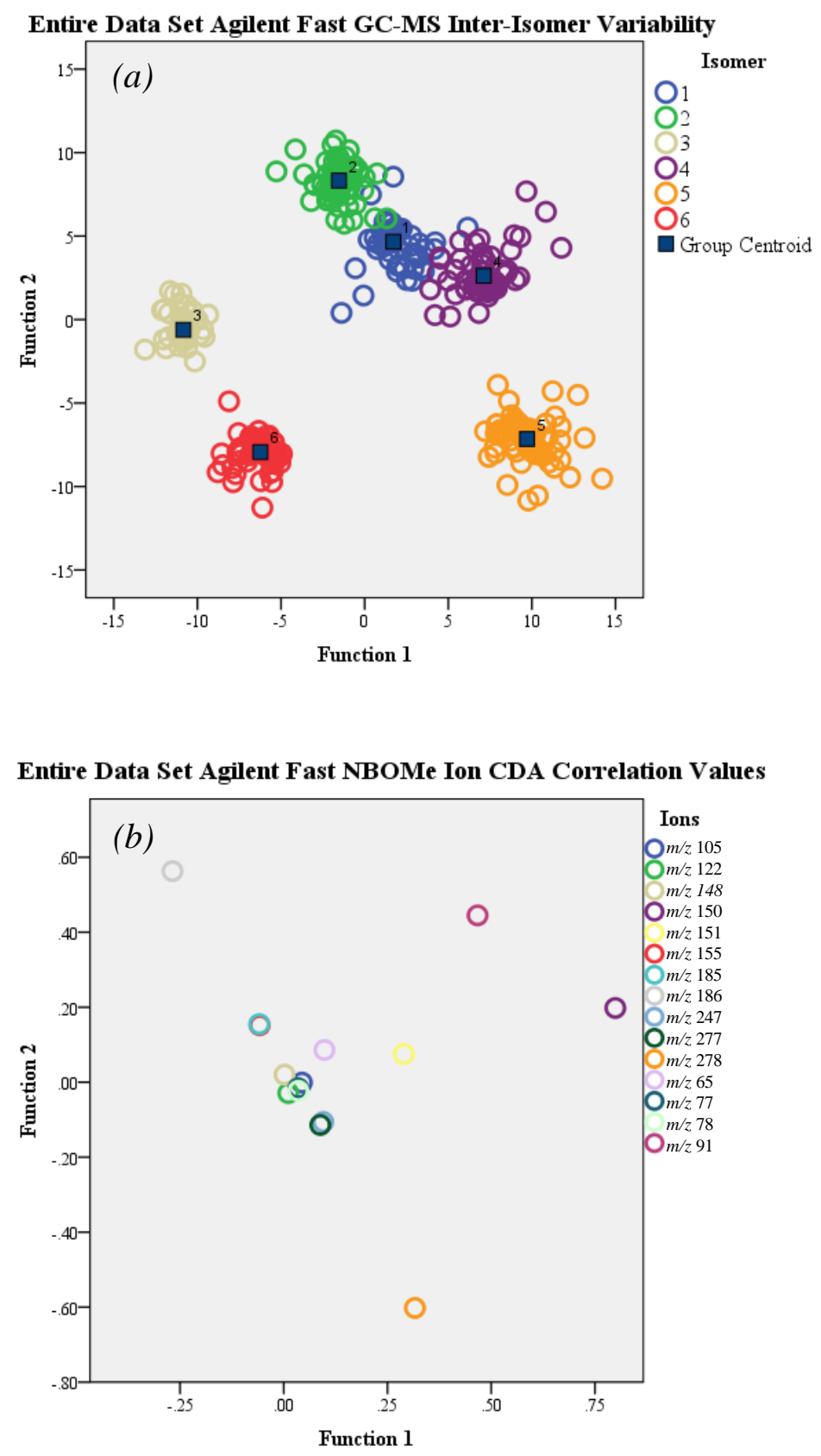

Figure 3.26. Canonical Discriminant Analysis (CDA) showing the classification of the NBOMe isomers based on the relative ion abundances for only the Agilent fast GC-MS methodology (a) and the structure matrix correlation values where function 1 and 2 are the pooled within group correlations between the discriminant variables and the standardized canonical discriminant functions (b). N=519 for the Agilent fast GC-MS entire data set. 
The CDA plot for the relative ion abundances for the premium data set generated for only the Agilent fast GC-MS methodology and the plot of the ion correlation values are shown in Figure 3.27. The CDA plot shows the classification of the relative ion abundances into six isomer groupings. The eigenvalue for function 1 is 415.8 and $52.7 \%$ of the variance is captured by this discriminant function. The eigenvalue for the second discriminant function is 203.2 and $25.8 \%$ of the variance is captured with this discriminant function. In total, $78.5 \%$ of the total variance is captured by the first two discriminant functions.

The variance for each isomer grouping relative to the group centroid is much smaller for the Agilent fast GC-MS premium data set in comparison to the entire Agilent fast GC-MS data set. Additionally, the ion correlation plot for the Agilent fast GC-MS premium data set has a wider spread of the correlation values than the entire data set ion correlation plot. Again, the relationship between the ion correlations and isomer groupings is apparent as the $25 \mathrm{C}-\mathrm{NBOMe}$ and 25I-NBOMe positional isomers are separated in relation to the $\mathrm{m} / \mathrm{z} 186$ and $\mathrm{m} / \mathrm{z} 278$ ions and the ortho, meta, and para isomers of both NBOMe compounds are separated in relation to the $\mathrm{m} / \mathrm{z}$ 91 and $\mathrm{m} / z$ 150. However, $\mathrm{m} / z 155$ and $\mathrm{m} / z 185$ show a stronger correlation to the separation of NBOMe isomers by relative ion abundance than previously observed.

The classification results based on the predicted group membership for both the original grouped and the cross-validated grouped cases were $100.0 \%$. This is an improvement from the Agilent fast GC-MS entire data set classification and is consistent with previous results which indicate that the classification rate increases when the higher concentration, or less variable intraisomer relative ion abundances are used for the classification of NBOMe isomers. 


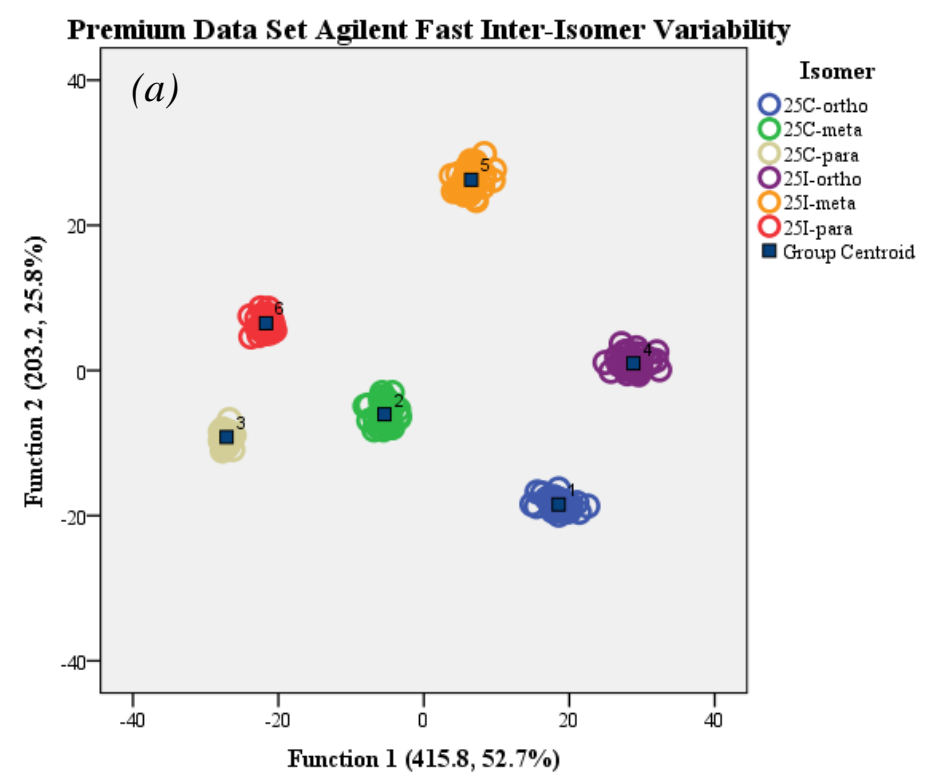

Premium Data Set Agilent Fast NBOMe Ion CDA Correlation Values

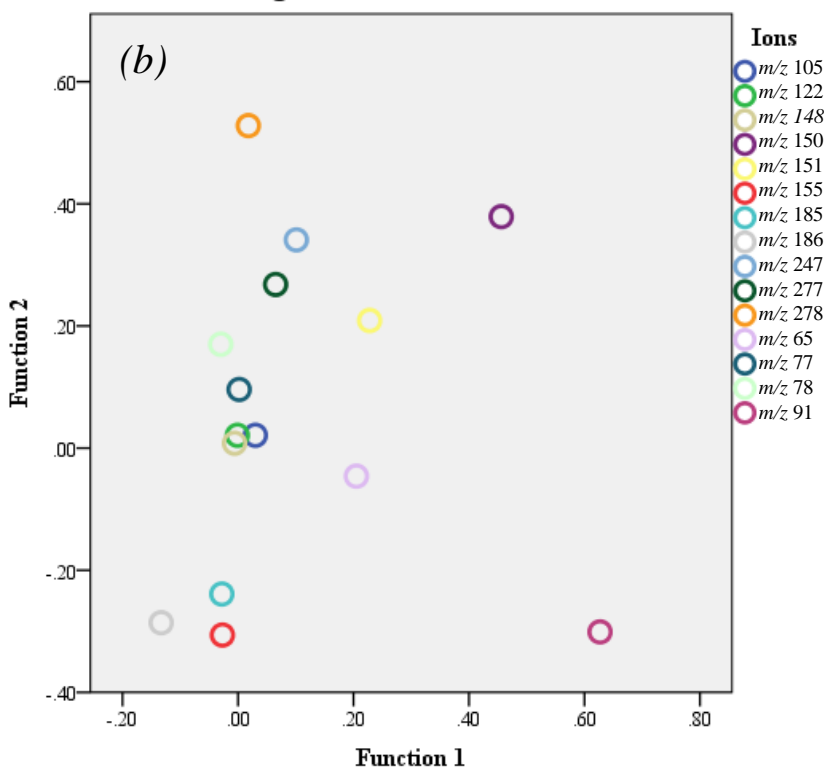

Figure 3.27. Canonical Discriminant Analysis (CDA) showing the classification of the NBOMe isomers based on the relative ion abundances for only the Agilent fast GC-MS methodology (a) and the structure matrix correlation values where function 1 and 2 are the pooled within group correlations between the discriminant variables and the standardized canonical discriminant functions (b). N=293 for the Agilent fast GC-MS premium data set, which contains the $125 \mathrm{ppm}$ and 1,250 ppm samples. 
Table 3.11 shows the results for the $95 \%$ confidence interval for the characteristic ion ratios proposed through this project. The four ions present were selected from a list of a possible 15 ions based on their relative ion abundance to the base peak at $\mathrm{m} / \mathrm{z}, 121$ and the significance of their inclusion for the differentiation of the six isomers studied during this project. The results were grouped together between concentration and instrumentation even though differences were identified with ANOVA analysis. Even though differences were identified in the idealized data, without grouped results this information provides less assistance to crime labs. In a crime lab, the exact performance of the mass spectrometer and the concentration of the unknown sample are not known. Thus, it is important to provide relative ion abundance results across all platforms analyzed during this experiment.

Table 3.11. 95\% confidence interval characteristic ion ratios.

\begin{tabular}{|c|c|c|c|c|}
\hline \multirow{2}{*}{ Isomer } & \multicolumn{5}{|l|}{$\mathbf{9 5}$ C.I. } & $\boldsymbol{m} / \boldsymbol{z} \mathbf{1 5 0}$ & $\boldsymbol{m} / \boldsymbol{z} \mathbf{1 8 6}$ & $\boldsymbol{m} / \boldsymbol{z} \mathbf{2 7 8}$ \\
\cline { 2 - 5 } & $\boldsymbol{m} / \boldsymbol{z} \mathbf{9 1}$ & $* 43.75 \pm 0.54$ & $* 1.94 \pm 0.11$ & $0.002 \pm 0.003$ \\
\hline 25C-ortho & $31.27 \pm 0.54$ & $* 55.65 \pm 0.58$ & $* 9.21 \pm 0.24$ & $0.008 \pm 0.001$ \\
\hline 25C-meta & $* 12.19 \pm 0.27$ & $* 16.15 \pm 0.26$ & $* 4.28 \pm 0.11$ & $0.001 \pm 0.002$ \\
\hline 25C-para & $* 4.33 \pm 0.15$ & $* 59.62 \pm 0.74$ & $0.01 \pm 0.01$ & $* 2.53 \pm 0.17$ \\
\hline 25I-ortho & $31.11 \pm 0.62$ & $* 70.11 \pm 0.86$ & $0.03 \pm 0.03$ & $* 14.35 \pm 0.58$ \\
\hline 25I-meta & $* 13.23 \pm 0.41$ & $* 21.25 \pm 0.32$ & $0.07 \pm 0.01$ & $* 6.72 \pm 0.28$ \\
\hline 25I-para & $* 4.92 \pm 0.34$ &
\end{tabular}

* Indicates the number is statistically significantly different at the $95 \%$ confidence interval between isomers.

Ions $m / z, 186$ and $m / z 278$ are proposed to be characteristic ion ratios because they are specific to 25C-NBOMe and 25I-NBOMe, respectively. The presence of either $\mathrm{m} / \mathrm{z} 278$ or $\mathrm{m} / \mathrm{z}$ 186 cannot only be used to identify which NBOMe is present, but the positional isomers can be differentiated based on the relative abundance as well. The correlation plot for the premium CDA data shows the large correlation between $\mathrm{m} / \mathrm{z} 186$ and $\mathrm{m} / \mathrm{z}, 278$ for isomer differentiation. 
The two ions with the most significant impact on the differentiation of the six NBOMe isomers analyzed are $\mathrm{m} / \mathrm{z}, 91$ and $\mathrm{m} / \mathrm{z}, 150$. These are the two most abundant fragments, with the exception of the base peak at $m / z 121$, and they are shown to have strong correlations to isomer differentiation in the entire NBOMe data set and the premium NBOMe data set. The relative abundance of $\mathrm{m} / \mathrm{z}, 150$ appears to be the most important relative ion ratio for the differentiation of these six NBOMe isomers with regard to the mass spectral data based on the PCA and CDA results. 
Figure 3.28 shows the $95 \%$ confidence interval relative ion abundances of the four proposed characteristic ion ratios calculated using the entire data set, where the values that are statistically significantly different at the $95 \%$ confidence interval based on a t-test are indicated. While all six NBOMe isomers can be differentiated with the relative ion abundance of $\mathrm{m} / \mathrm{z} 150$, the use of the three additional relative ion abundances adds additional value to the isomer differentiation.

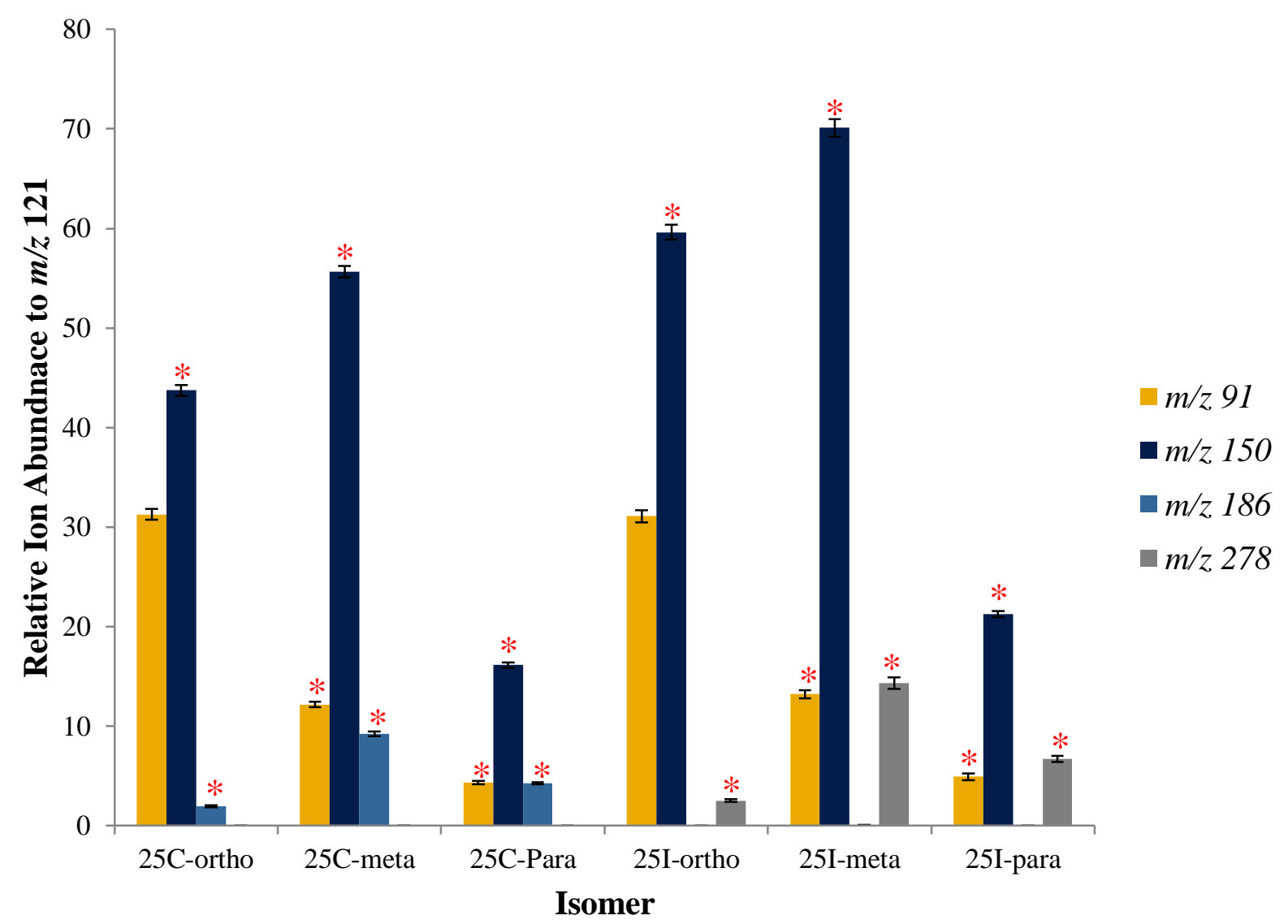

Figure 3.28. 95\% confidence interval for the characteristic ion ratios between the six NBOMe isomers studied. *Indicates statistically significantly different based on t-test results for each $\mathrm{m} / z$ value between isomers. 
Figure 3.29 shows the box plots for the relative ion abundance of the four characteristic ions proposed during this research project from the entire data set. The median values are indicated by the black line at the center of the box, with the minimum and maximum values located at the ends of the whiskers. The outliers that were present in the data were removed for demonstration purposes. The differentiation of the six NBOMe isomers is possible with these four characteristic ion ratios.
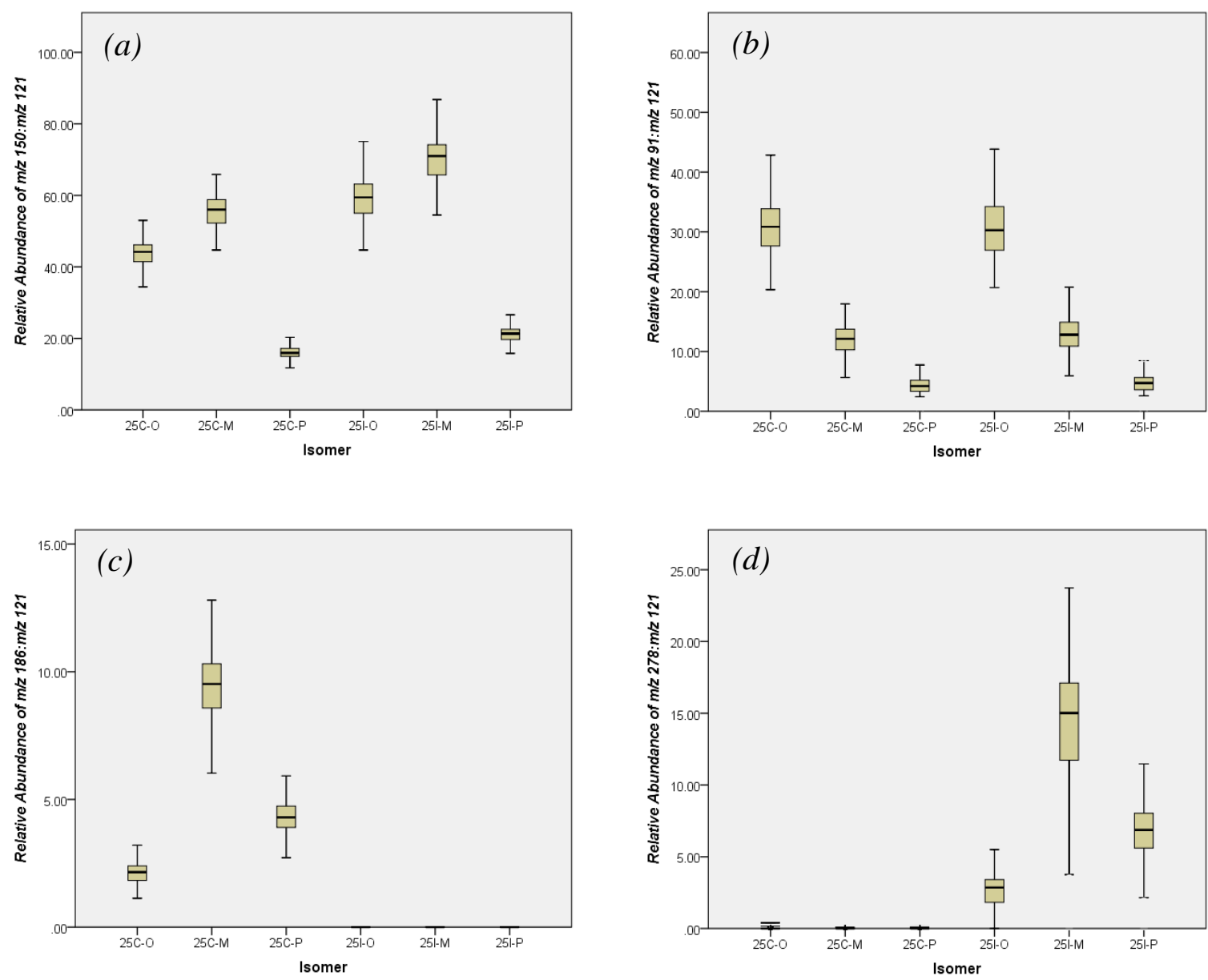

Figure 3.29. Box and whisker plots of the relative ion abundances for the four characteristic ion ratios proposed through this research, which are $\mathrm{m} / \mathrm{z} 150(\mathrm{a}), \mathrm{m} / \mathrm{z}, 91(\mathrm{~b})$, $m / z 186(c)$, and $m / z 278(d)$. 


\section{Conclusions}

This research demonstrates that conventional labs, using traditional instruments, can accomplish significant time savings without significant loss in performance with the use of fast GC-MS. The conversion process can be completed with ease and requires no additional education or instrument modifications outside the replacement of the column, which must be done routinely anyway. Based on the chromatographic separation efficiency results, it is concluded that the Agilent fast GC-MS results are not statistically different from the Agilent traditional GC-MS results with regard to the resolution and number of theoretical plates, yet the analyses were completed in half the time. Likewise, when normalized to n-alkanes, the Agilent fast GC-MS results produced highly reproducible results with the smallest \% RSD of the three techniques studied.

The determination of distinct retention indices and characteristic ion ratios is a significant contribution to the knowledge about NBOMe isomers. The ability to differentiate between NBOMe isomers through the use of retention indices allows crime labs using different instrumental setups and parameters to differentiate between these six NBOMe isomers. Additionally, this study demonstrates that the retention time match windows that many organizations recommend, such as the $\pm 2 \%$ RSD or \pm 0.1 minute retention time difference, may be too lenient.

The identification of four characteristic ion ratios that allow for the differentiation of these six NBOMe isomers is a significant contribution to the knowledge about NBOMe isomers. These results indicate that only the relative abundance of a single ion $(\mathrm{m} / \mathrm{z}, 150)$ is required to identify the isomer present and the inclusion of any of the additionally proposed characteristic ion ratios only further provides evidence to the identification of the isomer. Likewise, the 
ANOVA results indicate that concentration plays a significant role on the relative ion abundance of all ions except $m / z 91$ and $m / z$ 150, which indicates a possible quantitative relationship present in the relative ion abundances that could be further explored in an additional study.

The inclusion of the classification study performed with the CDA relative ion abundance results indicates that at higher concentrations, where less variability is present, the classification of NBOMe isomers increased to near perfect classification. However, even when low concentration samples were included, the classification rate was still 99.5\%. The use of multivariate classification shows great promise for the differentiation and possible identification of similar chemical structures based on the mass spectral fragmentation.

Finally, the identification of the significance of different factors on the within-factor to between-factor variance of the retention times, retention indices, and relative ion abundances contributes to the greater knowledge about GC-MS instrumental variability and also to crime lab quality assurance. The determination that the tune profile does not make a significant difference on the relative ion abundances is significant to crime labs today as it helps to answer the questions associated with the frequency and significance of autotunes performed within the lab. By providing this information to crime labs there is evidence that suggests that the set tune schedules performed by crime labs do not have a significant effect on the results being obtained and two analyses performed under different tune profiles are not significantly different in relative ion abundance at the $95 \%$ confidence level.

\section{Future Work}

Now that these characteristic ion ratios have been generated, a comparison to external sources is the next logical step. This comparison should include both NBOMe isomers from national databases, such as the NIST or Wiley databases, as well as standards from crime labs 
across the United States. If the characteristic ion ratios are observed from both of these sources, then the proposed characteristic ion ratios should be applied to casework samples. Potentially, this could also involve quantitative work with the relative ion abundances that showed significant differences at the $95 \%$ confidence level. It must be noted that the use of casework samples eliminates the possibility of a ground truth and thus, would be more exploratory in nature. For purposes of quantitation, these studies would need to be performed with NBOMe standards.

The next step would involve an analysis of the proposed characteristic ion ratios to that of other $2 \mathrm{C}$ compounds. This comparison could be from national databases or standards analyzed by crime labs. While the mass to charge ratios may not be conserved between other $2 \mathrm{C}$ drugs, there may be a link between relative ion abundance of the major ions present in the spectrum. For example, the ions with the highest discriminating power for NBOMes occur at relative ion abundances between 10 and 60 percent. This may also hold true with other $2 \mathrm{C}$ drugs, allowing for isomer differentiation based on the relative abundances of the ions that fall within these relative abundance bins.

The application of multivariate classification for the differentiation and identification of NBOMe isomers was explored. In this study, the CDA classification was very successful at differentiating between these six NBOMe isomers, but it would be useful to increase the diversity of samples analyzed. The inclusion of a non-halogenated NBOMe, such as $25 \mathrm{H}-$ NBOMe, and additionally substituted NBOMes would allow for exploration into the effects of additional substitutions on the substance fragmentation and resulting multivariate classification. 


\section{References}

1. Poklis, J.L., et al., Analysis of 25I-NBOMe, 25B-NBOMe, 25C-NBOMe and Other Dimethoxyphenyl-N-[(2-Methoxyphenyl) Methyl]Ethanamine Derivatives on Blotter Paper. J. Anal. Toxicol., 2015. 39(8): p. 617-23.

2. Casale, J.F. and P.A. Hays, Characterization of Eleven 2,5-Dimethoxy-N-(2methoxybenzyl)phenethylamine (NBOMe) Derivatives and Differentiation from their 3and 4-Methoxybenzyl Analogues - Part I. Microgram. 9(2): p. 84-109.

3. Stellpflug, S.J., et al., 2-(4-Iodo-2,5-dimethoxyphenyl)-N-[(2methoxyphenyl)methyl]ethanamine (25I-NBOMe): clinical case with unique confirmatory testing. J. Med. Toxicol., 2014. 10(1): p. 45-50.

4. Nikolau, P., et al., Beware of 25C-NBOMe: an N-benzyl substituted Phenethylamine. $J$. Forensic Toxicol. Pharmacol., 2014. 3(3).

5. Drug Enforcement Administration, Schedules of Controlled Substances: Placement of Three Synthetic Phenethylamines Into Schedule I, in Fed. Regist. 2013.

6. Drug Enforcement Administration, Schedules of Controlled Substances: Extension of Temporary Placement of Three Synthetic Phenethylamines in Schedule I. Final order., in Fed. Regist. 2015.

7. Marek, G.J. and G.K. Aghajanian, LSD and the phenethylamine hallucinogen DOI are potent partial agonists at 5-HT2A receptors on interneurons in rat piriform cortex. $J$. Pharm. Exp. Ther. 278(3): p. 1373-1382.

8. Sánchez Robayo, D., A,, et al., Analysis of Blotter Papers Employed in the Commercialization of New Hallucinogenic Substances of the 2,5-Dimethoxy-N-(2methoxybenzyl) Phenethylamine Series Seized in the City of Bogotá by Applying Gas Chromatography Coupled to a Selective Mass Ion Trap Detector. J. Braz. Chem. Soc., 2016.

9. Stuenkel, C.A., R.E. Dudley, and S.S. Yen, Sublingual administration of testosteronehydroxypropyl-beta-cyclodextrin inclusion complex simulates episodic androgen release in hypogonadal men. J. Clin endocrinol. Metaab., 1991. 72(5): p. 1054-1059.

10. Erowid Experience Vault. 25I-NBOMe [cited 2015 November 16]; Available from: http://www.erowid.org/chemicals/2ci_nbome.

11. Hill S. L, et al., Severe Clinical Toxicity Associated with Analytically Confirmed Recreational Use of 25I-NBOMe: Case Series. J. Clin. Toxicol., 2013. 51: p. 487-492.

12. Emolecules, Chemical Drawing Service.

13. Sigma-Aldrich, A Practical Guide for Increasing Sample Throughput without Sacrificing Quality.

14. Klee, M.S. and L.M. Blumberg, Theoretical and Practical Aspects of Fast Gas Chromatography and Method Translation. J. Chromatogr. Sci., 2002. 40: p. 234-247.

15. Maštovská, K. and S.J. Lehotay, Practical approaches to fast gas chromatography-mass spectrometry. J. Chromatogr. A, 2003. 1000(1-2): p. 153-180.

16. Skoog, D., J. Holler, and S. Crouch, Principles of Instrumental Analysis: An Introduction to Chromatographic Separations. 6th ed. 2007, Canada: Thomson Brooks/Cole.

17. Lum, B.J., J.J. Brophy, and D.B. Hibbert, Identification of 4-substituted 2-(4-x-2,5dimethoxyphenyl)-N-[(2-methoxyphenyl)methyl]ethanamine (25X-NBOMe) and analogues by gas chromatography-mass spectrometry analysis of heptafluorobutyric anhydride (HFBA) derivatives. Aust. J. Forensic Sci., 2015. 48(1): p. 59-73. 
18. Poklis, J.L., et al., Postmortem detection of 25I-NBOMe [2-(4-iodo-2,5dimethoxyphenyl)-N-[(2-methoxyphenyl)methyl]ethanamine] in fluids and tissues determined by high performance liquid chromatography with tandem mass spectrometry from a traumatic death. Forensic Sci. Int., 2014. 234: p. e14-20.

19. Poklis, J.L., et al., High-performance liquid chromatography tandem mass spectrometry method for the determination of 2CC-NBOMe and 25I-NBOMe in human serum. $J$. Biomed Chromatogr., 2013. 27(12): p. 1794-800.

20. Uchiyama, N., et al., Two new synthetic cannabinoids, AM-2201 benzimidazole analog (FUBIMINA) and (4-methylpiperazin-1-yl)(1-pentyl-1H-indol-3-yl)methanone (MEPIRAPIM), and three phenethylamine derivatives, 25H-NBOMe 3,4,5trimethoxybenzyl analog, 25B-NBOMe, and 2C-N-NBOMe, identified in illegal products. J. Forensic Toxicol., 2014. 32(1): p. 105-115.

21. Uchiyama, N., et al., URB-754: a new class of designer drug and 12 synthetic cannabinoids detected in illegal products. Forensic Sci. Int., 2013. 227(1-3): p. 21-32.

22. Coelho Neto, J., Rapid detection of NBOME's and other NPS on blotter papers by direct ATR-FTIR spectrometry. Forensic Sci. Int., 2015. 252: p. 87-92.

23. Duffau, B., et al., Analysis of 25 C NBOMe in Seized Blotters by HPTLC and GC-MS. $J$ Chromatogr. Sci., 2016. 54(7): p. 1153-8.

24. Salomone, A., et al., Determination of cathinones and other stimulant, psychedelic, and dissociative designer drugs in real hair samples. Anal. Bioanal. Chem., 2016. 408: p. 2035-2042.

25. Agilent Technologies. VF-5MS Capillary Column [cited 2016 January 9]; Available from: http://www.agilent.com/en-us/products/gas-chromatography/gccolumns/capillary/vf-5ms\#buy-parts.

26. Elie, M.P., L.E. Elie, and M.G. Baron, Keeping pace with NPS releases: fast GC-MS screening of legal high products. Drug. Test. Anal., 2013. 5(5): p. 281-90.

27. Strano-Rossi, S., et al., Fast GC-MS method for the simultaneous screening of THC$\mathrm{COOH}$, cocaine, opiates and analogues including buprenorphine and fentanyl, and their metabolites in urine. Anal. Bioanal. Chem., 2011. 399(4): p. 1623-30.

28. Analysis of Variance-online statistics. [cited 2016 February 11]; Available from: http://onlinestatbook.com/2/analysis_of_variance/anova.pdf.

29. Smith, L.I., A Tutorial on Principle Components Analysis. p. 1-27.

30. J.G. Cruz-Castillo, S.G., B.R. MacKay, G.S. Lawes, C.R.O. Lawoko, and D.J. Woolley, Applications of Canonical Discriminant Analysis inHorticultural Research. J. Hortic. Sci., 1994. 29(10): p. 1115-1119.

31. Van Den Dool, H., Kratz, P.D., J. Chromatogr. 1963. 11: p. 463-471.

32. Bodnar Willard, M.A., R. Waddell Smith, and V.L. McGuffin, Statistical approach to establish equivalence of unabbreviated mass spectra. Rapid Commun. Mass Spectrom., 2014. 28(1): p. 83-95.

33. Industriestrasse, M.K., Gas Chromatography Troubleshooting and Reference Guide. 2005.

34. Agilent Technologies. GC Resolution [cited 2017 March 7]; Available from: http:// www.chem.agilent.com.

35. Rigdon, A., The Forgotten Septum. Restek GC Accessories, 2008. 1.

36. Lane, D.M., One-Factor ANOVA (Between Subjects). Onlinestatbook.com.

37. George Washington University, Hypothesis Testing and ANOVA. 
38. Agilent Technologies, What are the major Causes of GC Capillary Column Performance Degradation? 2007: p. 4.

39. Richardson, J.T.E., Eta squared and partial eta squared as measures of effect size in educational research. Educational Research Review, 2011. 6(2): p. 135-147.

40. United Nations Office on Drugs and Crime, Guidance for the Validation of Analytical Methodology and Calibration of Equipment used for Testing of Illicit Drugs in Seized Materials and Biological Specimens.

41. Agilent Technologies, Validation of Analytical Methods.

42. European Commission, Safety of the Food Chain Pesticides and Biocides.

43. Lehotay, S.J., USDA-FSIS: Update of Qualitative Analysis Considerations in GC$M S(/ M S)$.

44. Zuba, D. and K. Sekula, Analytical characterization of three hallucinogenic N-(2methoxy)benzyl derivatives of the 2C-series of phenethylamine drugs. Drug. Test. Anal., 2013. 5(8): p. 634-45.

45. Harris, D.N., et al., Fragmentation differences in the EI spectra of three synthetic cannabinoid positional isomers: JWH-250, JWH-302, and JWH-201. Int. J. Mass Spectrom., 2014. 368: p. 23-29.

46. Agilent Technologies 5977 Series MSD: Operation Manual. 